\title{
Search for direct production of supersymmetric partners of the top quark in the all-jets final state in proton-proton collisions at $\sqrt{s}=13 \mathrm{TeV}$
}

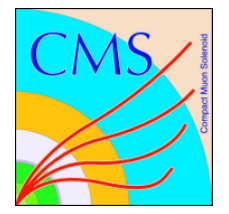

\section{The CMS collaboration}

E-mail: cms-publication-committee-chair@cern.ch

ABSTRACT: A search for direct production of top squark pairs in events with jets and large transverse momentum imbalance is presented. The data are based on proton-proton collisions at a center-of-mass energy of $13 \mathrm{TeV}$, collected with the CMS detector in 2016 at the CERN LHC, and correspond to an integrated luminosity of $35.9 \mathrm{fb}^{-1}$. The search considers a variety of $R$-parity conserving supersymmetric models, including ones for which the top squark and neutralino masses are nearly degenerate. Specialized jet reconstruction tools are developed to exploit the unique characteristics of the signal topologies. With no significant excess of events observed above the standard model expectations, upper limits are set on the direct top squark pair production cross section in the context of simplified supersymmetric models for various decay hypotheses. Models with larger differences in mass between the top squark and neutralino are probed for masses up to 1040 and $500 \mathrm{GeV}$, respectively, whereas models with a more compressed mass hierarchy are probed up to 660 and $610 \mathrm{GeV}$, respectively. The smallest mass difference probed is for masses near to 550 and $540 \mathrm{GeV}$, respectively.

KEYWORDS: Hadron-Hadron scattering (experiments), Supersymmetry

ArXIV EPRINT: 1707.03316 


\section{Contents}

1 Introduction 1

2 The CMS detector 4

3 Simulated events $\quad 4$

4 Event reconstruction $\quad \mathbf{5}$

4.1 Identification of high- $p_{\mathrm{T}}$ top quarks and $\mathrm{W}$ bosons $\quad 7$

$\begin{array}{llr}4.2 & \text { Identification of intermediate- } p_{\mathrm{T}} \text { top quarks } & 9\end{array}$

$\begin{array}{lll}4.3 & \text { Identification of initial-state radiation } & 10\end{array}$

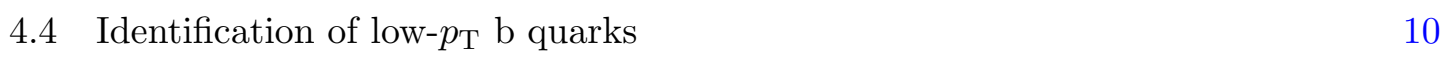

5 Search strategy $\quad 11$

5.1 Strategy for high $\Delta m$ models $\quad 11$

$\begin{array}{lll}5.2 & \text { Strategy for low } \Delta m \text { models } & 12\end{array}$

$\begin{array}{llr}6 & \text { Background estimation } & \mathbf{1 3}\end{array}$

$\begin{array}{lll}6.1 & \text { Estimation of the lost-lepton background } & 14\end{array}$

\begin{tabular}{ll}
6.2 & Estimation of the $\mathrm{Z}(\nu \nu)$ background \\
\hline
\end{tabular}

$\begin{array}{lll}\text { 6.3 Estimation of the QCD multijet background } & 17\end{array}$

$\begin{array}{ll}\text { 6.4 Estimation of "rare" SM backgrounds } & 18\end{array}$

$\begin{array}{ll}\text { 6.5 Validation of the background methods in data } & 18\end{array}$

$\begin{array}{llr}7 & \text { Systematic uncertainties } & 19\end{array}$

8 Results and interpretation $\quad 23$

$\begin{array}{lll}9 & \text { Summary } & 35\end{array}$

$\begin{array}{ll}\text { The CMS collaboration } & 45\end{array}$

\section{Introduction}

Although the standard model (SM) of particle physics provides a remarkably accurate description of phenomena associated with the known elementary particles and their interactions, it leaves significant problems unresolved. It cannot, for instance, explain how the Higgs boson [1-6] can evade divergent quantum corrections, without very significant fine tuning $[7,8]$ of SM parameters, to allow it to have its mass at the weak scale [9-14]. Moreover, an abundance of cosmological observations, including the existence of dark matter, cannot be explained within the context of the SM alone [15-17]. 
Supersymmetry (SUSY) provides a theoretical framework that can address these questions. At its core, SUSY is a symmetry between fermions and bosons. In SUSY, a "sparticle" (generally referred to as a superpartner) is proposed for each SM particle with the same gauge quantum numbers but differing by one half-unit of spin and potentially in mass. The superpartners of the electroweak vector $\mathrm{W}$ and $\mathrm{Z}$ bosons and scalar Higgs boson mix to produce charged and neutral fermions referred to as charginos $\left(\widetilde{\chi}^{ \pm}\right)$and neutralinos $\left(\widetilde{\chi}^{0}\right)$, respectively. For a given fermion $\mathrm{f}$, there are two superpartners corresponding to the fermion's left- and right-handed states. The superpartners mix to form two mass eigenstates, $\widetilde{\mathrm{f}}_{1}$ and $\widetilde{\mathrm{f}}_{2}$, with $\widetilde{\mathrm{f}}_{1}$ being the lighter of the two. The quantum corrections to the value of the Higgs boson mass $\left(m_{\mathrm{H}}\right)$ from sparticles could cancel the otherwise problematic SM contributions. In this way, SUSY can protect the value of $m_{\mathrm{H}}$ [18-21], provided that the mass differences between the SM particles and their superpartners are not too large. This is particularly important for superpartners of third generation SM particles, because they have the largest couplings to the Higgs boson, and therefore produce the largest corrections. Furthermore, a combination of precision measurements and null search results indicate that the superpartners of the light quarks may have very large masses [22]. In view of these considerations, the superpartners of the top and bottom quarks, the $\widetilde{t}$ and $\widetilde{\mathrm{b}}$ squarks, respectively, are expected to be among the lightest sparticles, potentially light enough to be produced at the CERN LHC [23]. An important point to note is that SUSY models with $R$-parity conservation $[24,25]$ require sparticles to be produced in pairs, with the lightest SUSY particle (LSP) therefore stable on cosmological time scales. This means that if the lightest neutralino, denoted $\widetilde{\chi}_{1}^{0}$, is the LSP, then it is also a very promising dark matter candidate [26] that would remain at the end of all $R$-parity conserving sparticle cascade decays. The two motivating principles above place the search for pair production of top squarks $(\widetilde{\mathrm{tt}})$ among the highest priorities of the LHC program.

The most recent searches for direct $\widetilde{\bar{t}}$ production were carried out by the ATLAS and CMS Collaborations in proton-proton (pp) collisions at center-of-mass energies $\sqrt{s}$ of 7 , 8, and $13 \mathrm{TeV}$ at the LHC [27-47]. The searches have provided no evidence for sparticle production in models with $\widetilde{\mathrm{t}}$ masses up to $\sim 900 \mathrm{GeV}$ and $\widetilde{\chi}_{1}^{0}$ masses up to $\sim 400 \mathrm{GeV}$.

This paper presents a search for direct $\overline{\mathrm{tt}}$ production in $R$-parity conserving SUSY using data collected in pp collisions at $\sqrt{s}=13 \mathrm{TeV}$ by the CMS experiment at the LHC in 2016, and corresponding to an integrated luminosity of $35.9 \mathrm{fb}^{-1}$. The search is based on methods presented in ref. [44], and represents an extension of that search to larger sparticle masses by means of a significantly larger dataset and the development of more sensitive search tools. This search focuses on all-hadronic final states, defined as those events whose visible content is made up solely of hadronic jets, as would be expected for signal processes in which all $\mathrm{W}$ bosons decay to quarks. These final states have the largest accessible branching fraction. In many SUSY models, the favored $\widetilde{t}$ decay modes depend strongly on the mass hierarchy of the sparticles. In particular, different ranges of mass difference $\Delta m$ between the $\widetilde{t}$ and $\widetilde{\chi}_{1}^{0}$ correspond to very different final-state signatures. Only the lightest $\widetilde{t}$ mass eigenstate, $\widetilde{t}_{1}$, is assumed to be involved in the models considered in this paper, although the results are expected to be equivalent for the heavier eigenstate. 


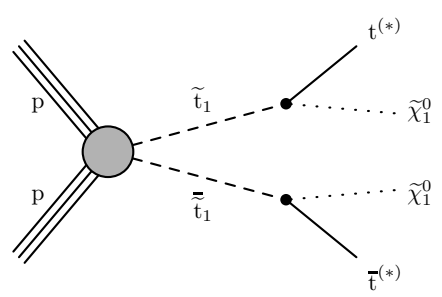

(a)

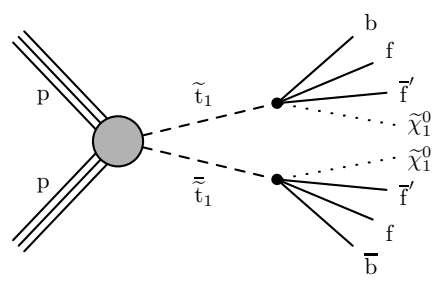

(d)

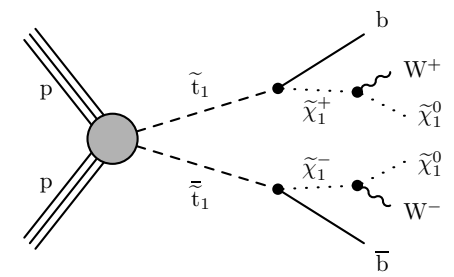

(b)

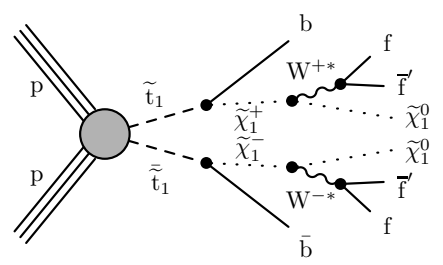

(e)

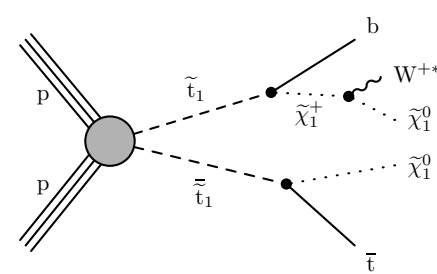

(c)

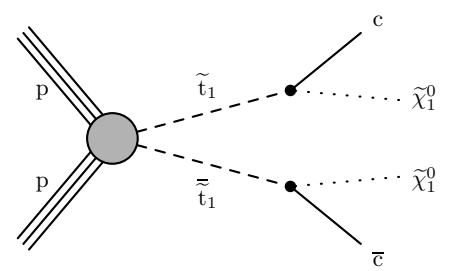

(f)

Figure 1. Diagrams for the decay modes of pair-produced top squarks studied in this analysis. The decay cascades are denoted: (a) T2tt, (b) T2bW, (c) T2tb, (d) T2ttC, (e) T2bWC, and (f) T2cc. An asterisk indicates that the particle may be produced off-shell.

The $\widetilde{t}_{1}$ decay modes of the simplified models [48-50] that are used as the basis for our searches are displayed in figure 1 .

The search regions (SR) are optimized for different models and ranges of $\Delta m$. In models with $\Delta m$ larger than the $\mathrm{W}$ boson mass $m_{\mathrm{W}}$ ("high $\Delta m$ models"), the simplest decays that we consider are $\widetilde{\mathrm{t}}_{1} \rightarrow \mathrm{t}^{(*)} \widetilde{\chi}_{1}^{0}$, denoted "T2tt", and $\widetilde{\mathrm{t}}_{1} \rightarrow \mathrm{b} \widetilde{\chi}_{1}^{ \pm} \rightarrow \mathrm{bW} \mathrm{W}^{ \pm} \widetilde{\chi}_{1}^{0}$, denoted "T2bW", under the assumption that the $\widetilde{\chi}_{1}^{ \pm}$mass lies halfway between the $\widetilde{t}_{1}$ and $\widetilde{\chi}_{1}^{0}$ masses. The choice of moderate $\widetilde{\chi}_{1}^{ \pm}$mass in the latter model permits high momentum objects in the final state. The $\widetilde{\chi}_{1}^{ \pm}$represents the lightest chargino, and $\widetilde{\chi}_{1}^{0}$ is the stable LSP, which escapes detection to produce a large transverse momentum imbalance in the event. Another model, denoted "T2tb", is considered under the assumption of equal branching fractions of the two aforementioned decay modes. This model, however, assumes a compressed mass spectrum in which the mass of the $\widetilde{\chi}_{1}^{ \pm}$is only $5 \mathrm{GeV}$ greater than that of the $\widetilde{\chi}_{1}^{0}$. As a result, the $\mathrm{W}$ bosons from chargino decays are produced far off-shell.

In models with $\Delta m$ less than $m_{\mathrm{W}}$ ("low $\Delta m$ models"), the $\widetilde{\mathrm{t}}_{1}$ can decay through the T2tt decay mode with off-shell $\mathrm{t}$ and $\mathrm{W}$, through the same decay chain as in the T2bW model, via off-shell $\mathrm{W}$ bosons, or decay through a flavor changing neutral current process $\left(\widetilde{\mathrm{t}}_{1} \rightarrow \mathrm{c} \widetilde{\chi}_{1}^{0}\right.$, where $\mathrm{c}$ is the charm quark). These will be referred to as the "T2ttC", "T2bWC", and "T2cc" models, respectively, where C denotes the hypothesis of a compressed mass spectrum in the first two cases. Observations in such low $\Delta m$ models are experimentally challenging since the visible decay products are typically very soft (low-momentum), and therefore often evade identification. Nevertheless, such models are particularly interesting because their dark matter relic density is predicted to be consistent with the cosmological observations [51]. Specialized jet reconstruction tools and event selection criteria are therefore developed to enhance sensitivity to these signals. 
This paper is organized as follows. A brief description of the CMS detector is presented in section 2, while section 3 discusses the simulation of background and signal processes. Event reconstruction is presented in section 4, followed by a description of the search strategy in section 5. Methods employed to estimate the SM backgrounds and their corresponding systematic uncertainties are detailed in sections 6 and 7, respectively. The discussion of the systematic uncertainties assigned to the signal processes is also presented in section 7. The results of the search and their interpretation in the context of a variety of models of $\widetilde{t}_{1}$ production and decay are presented in detail in section 8 , followed by a summary in section 9 .

\section{The CMS detector}

The central feature of the CMS apparatus is a superconducting solenoid of $6 \mathrm{~m}$ internal diameter, providing a magnetic field of 3.8 T. Within the solenoid volume are an all-silicon pixel and strip tracker, a lead tungstate crystal electromagnetic calorimeter (ECAL), and a brass and scintillator hadron calorimeter (HCAL), each composed of a barrel and two endcap sections. Forward calorimeters extend the pseudorapidity $(\eta)$ coverage provided by the barrel and endcap detectors. Muons are measured in gas-ionization detectors embedded in the steel flux-return yoke outside the solenoid. The first level of the CMS trigger system, composed of custom hardware processors, uses information from the calorimeters and muon detectors to select the most interesting events in a fixed time interval of less than $4 \mu \mathrm{s}$. The high-level trigger processor farm further decreases the event rate from around $100 \mathrm{kHz}$ to around $1 \mathrm{kHz}$, before data storage. A more detailed description of the CMS detector, together with a definition of the coordinate system used and the relevant kinematic variables, can be found in ref. [52].

\section{Simulated events}

Monte Carlo (MC) simulated events are used to study the important SM backgrounds, as well as to formulate the overall search for SUSY processes. Background processes composed uniquely of jets produced via the strong interaction of quantum chromodynamics (QCD) are referred to as "QCD multijet" processes. Simulated events originating from $\mathrm{t} \overline{\mathrm{t}}, \mathrm{W}+$ jets, $\mathrm{Z}+$ jets, $\gamma+$ jets, and $\mathrm{QCD}$ multijet processes are generated using MADGraph5_amC@NLO 2.3.3 [53] at leading order (LO) using the LO NNPDF3.0 [54] parton distribution functions $(\mathrm{PDF})$. The $\mathrm{WZ}, \mathrm{ZZ}, \mathrm{t} \overline{\mathrm{t} Z}$, and $\mathrm{t} \overline{\mathrm{t}} \mathrm{W}$ processes are generated using MADGRAPH5_AMC@NLO at next-to-leading order (NLO), the single top quark process in the tW channel using POWHEG [55-58] and the WW process is generated at NLO with POWHEG v2.0 [59], all using the NLO NNPDF3.0 PDF. In all of the aforementioned cases, parton showering and hadronization are simulated in PYTHIA 8.212 [60]. The potential for double counting of partons generated using PYTHIA with those using MAdGrAPH5_amC@NLO is minimized using the MLM [61] and the FXFX [62] matching schemes, in the LO and NLO samples, respectively. To evaluate systematic uncertainties associated with these aspects of event simulation, two additional t $\bar{t}$ samples are generated 
using POWHEG v2.0 [63], where one is interfaced with PYTHIA and the other with HER$\mathrm{WIG}++\mathrm{v} 2.7 .1$ [64]. Additional QCD multijet samples are also generated, but interfaced with HERWIG ++ for the modeling of parton showering and hadronization. Signal processes are generated at LO using MADGRAPH5_aMC@NLO based on the LO NNPDF3.0 PDF with PYTHIA used for parton showering and hadronization. Signal production cross sections are calculated using NLO with next-to-leading logarithm (NLL) soft-gluon resummations $(\mathrm{NLO}+\mathrm{NLL})[65]$. The most precise cross section calculations are used to normalize the SM simulated samples, corresponding to next-to-next-to-leading order (NNLO) accuracy [66$69]$ in most cases. Finally, the transverse momentum $\left(\vec{p}_{\mathrm{T}}\right.$, with magnitude $\left.p_{\mathrm{T}}\right)$ spectrum of top quarks in $\mathrm{t} \overline{\mathrm{t}}$ events is reweighted (referred to as "top quark $p_{\mathrm{T}}$ reweighting") to account for effects due to missing higher-order corrections in MC simulation, according to the results presented in ref. [70].

A full GEAnT4-based model [71] is used to simulate the response of the CMS detector to SM background samples. The CMS fast simulation package [72] is used for signal samples after verifying that it provides results that are consistent with those obtained from the full Geant4-based simulation. Event reconstruction is treated in the same manner for $\mathrm{MC}$ simulation as for data. A nominal distribution of multiple pp collisions in the same or neighboring bunch crossings (referred to as "pileup") is used to overlay the simulated events. The events are then reweighted to match the pileup profile observed in the collected data.

\section{Event reconstruction}

Events are reconstructed using the CMS particle-flow (PF) algorithm [73], which combines information from all detector subsystems to reconstruct the properties of the final-state particles produced in the pp collisions. At least one reconstructed vertex is required; for multiple collision vertices from pileup interactions, the reconstructed vertex with the largest value of summed physics-object $p_{\mathrm{T}}^{2}$ is taken to be the primary pp interaction vertex $(\mathrm{PV})$. The physics objects used in this context are the objects returned by a jet finding algorithm $[74,75]$ applied to all charged tracks associated with the vertex under consideration, plus the corresponding associated missing transverse momentum (the precise definition is given later in the text). Events affected by instrumental noise or reconstruction failures are identified through dedicated filters and rejected. Reconstructed particles are identified as charged hadrons, neutral hadrons, electrons, muons, or photons, to constitute a list of PF candidates.

Our primary jet collection is produced by clustering the PF candidates originating from the PV using the anti- $k_{\mathrm{T}}$ algorithm [74] with a distance parameter of 0.4. The jet energy is corrected for the contribution from pileup based on the jet area method [76, 77]. Additional corrections to the jet energy scale are applied to compensate for nonuniform detector response [78]. Jets are required to have $p_{\mathrm{T}} \geq 20 \mathrm{GeV}$ and be contained within the tracker volume of $|\eta| \leq 2.4$.

Jets originating from the hadronization of bottom (b) quarks are identified, or "tagged", through the combined secondary vertex (CSVv2) b tagging algorithm [79, 80]. The working point used provides an efficiency for the $b$ tagging of jets originating from 
b quarks that varies from 60 to $75 \%$ depending on $p_{\mathrm{T}}$, whereas the misidentification rate for light quarks or gluons is $\sim 1 \%$, and $\sim 15 \%$ for charm quarks. A novel soft $\mathrm{b}$ tagging algorithm was developed for this analysis and used to identify b quarks with $p_{\mathrm{T}}^{\mathrm{b}}<20 \mathrm{GeV}$ (i.e. below the jet $p_{\mathrm{T}}$ threshold). The algorithm is described in section 4.4. Although the T2cc model involves charm quark jets in the final state, no dedicated c tagger was used in this analysis.

To estimate the $p_{\mathrm{T}}$ imbalance in the event, the missing transverse momentum, $\vec{p}_{\mathrm{T}}^{\text {miss }}$, is defined as the negative of the vectorial sum of the $\vec{p}_{\mathrm{T}}$ of all PF candidates in the event. Its magnitude is denoted $p_{\mathrm{T}}^{\text {miss }}$. The jet energy scale corrections applied to the jets are propagated to $\vec{p}_{\mathrm{T}}^{\mathrm{miss}}$.

Electrons are reconstructed by combining information from the inner tracker with energy depositions in the ECAL [81]. Muons are reconstructed by combining tracks in the inner tracker and in the muon system [82]. Tracks associated with electrons or muons are required to originate from the PV, and a set of quality criteria is imposed to assure efficient identification $[81,82]$. To suppress misidentification of charged hadrons as leptons, we require electrons and muons to be isolated from jet activity within a $p_{\mathrm{T}}$-dependent cone size defined by a radius $R_{\text {rel }}$ in the $\eta-\phi$ plane, where $\phi$ is the azimuthal angle in radians. The relative isolation, $I_{\text {rel }}$, is defined as the scalar sum of the $p_{\mathrm{T}}$ of the PF candidates within the cone divided by the lepton $p_{\mathrm{T}}$. Charged $\mathrm{PF}$ candidates not originating from the $\mathrm{PV}$, as well as PF candidates identified as electrons or muons, are not considered in the sum. The cone size $R_{\text {rel }}$ depends on the lepton $p_{\mathrm{T}}$ :

$$
R_{\mathrm{rel}}= \begin{cases}0.2, & p_{\mathrm{T}}<50 \mathrm{GeV} \\ 10 \mathrm{GeV} / p_{\mathrm{T}}, & 50 \leq p_{\mathrm{T}}<200 \mathrm{GeV} \\ 0.05, & p_{\mathrm{T}} \geq 200 \mathrm{GeV}\end{cases}
$$

The decreasing cone radius at larger $p_{\mathrm{T}}$ provides high efficiency for the collimated decay products of highly Lorentz-boosted heavy objects [83]. The isolation sum $I_{\text {rel }}$ is corrected for contributions of neutral particles originated from pileup interactions using an area-based estimate [77] of pileup energy deposition in the cone.

Photons are reconstructed from energy depositions in the ECAL using identification algorithms that utilize a collection of variables related to the spatial distribution of shower energy in the supercluster (a group of 5x5 ECAL crystals), the photon isolation, and the fraction of the energy deposited in the HCAL behind the supercluster relative to the energy observed in the supercluster [84].

Tau lepton decays to hadrons, $\tau_{\mathrm{h}} \rightarrow$ (hadrons) $\nu_{\tau}$, are reconstructed starting from isolated charged-hadron candidates with $p_{\mathrm{T}} \geq 10 \mathrm{GeV}$ and $|\eta| \leq 2.4$. If there are photons with $p_{\mathrm{T}} \geq 0.5 \mathrm{GeV}$ within a cone of $R \leq 0.2$ around the charged hadron, the leading $p_{\mathrm{T}}$ photon momentum is vectorially added to that of the charged hadron candidate. In addition, we impose a requirement on the transverse mass of the $\tau_{\mathrm{h}}$; for an object with transverse momentum $\vec{p}_{\mathrm{T}}$, the transverse mass $m_{\mathrm{T}}$ is defined as:

$$
m_{\mathrm{T}}\left(\vec{p}_{\mathrm{T}}, \vec{p}_{\mathrm{T}}^{\mathrm{miss}}\right)=\sqrt{2 p_{\mathrm{T}} p_{\mathrm{T}}^{\text {miss }}(1-\cos \Delta \phi)}
$$


where $\Delta \phi$ is the difference in azimuthal angle between $\vec{p}_{\mathrm{T}}$ and $\vec{p}_{\mathrm{T}}^{\text {miss }}$. We require the transverse mass of the $\tau_{\mathrm{h}}$ to be less than $100 \mathrm{GeV}$, consistent with the expectation from a $\tau_{\mathrm{h}}$ emitted in a $\mathrm{W}$ boson decay in a high-multiplicity jet environment. A multivariate boosted decision tree (BDT) classifier [85] is trained to distinguish $\tau_{\mathrm{h}}$ decay products from other charged hadrons. Input variables include isolation sums within cones of several radii, $R$-distances from the $\tau_{\mathrm{h}}$ candidate to the nearest charged particle and to the axis of the jet in which it is contained, and the $\mathrm{b}$ tagging discriminant value of that jet.

Many of the $\tilde{t}_{1}$ decay modes involve unique final-state signatures. In view of this, reconstruction tools have been developed to exploit these signatures while significantly suppressing the SM background. Signal models with large $\Delta m$ have decay chains involving on-shell top quarks and $\mathrm{W}$ bosons. Identification of jets associated with the decays of top quarks and $\mathrm{W}$ bosons to quarks is an important component of the analysis, used to suppress most of the backgrounds in searches that target such signals. Because they exhibit a wide range of Lorentz boosts, we take different approaches in their reconstruction depending on whether they have large or small $p_{\mathrm{T}}$; these are described in sections 4.1 and 4.2 , respectively. In contrast, the decay products in models with small $\Delta m$ are very soft and often fail to be reconstructed through the standard algorithms. We have therefore developed more effective algorithms for these cases that are described in sections 4.3 and 4.4.

\subsection{Identification of high- $p_{\mathrm{T}}$ top quarks and $\mathrm{W}$ bosons}

The decay products of highly boosted top quarks with $p_{\mathrm{T}} \geq 400 \mathrm{GeV}$, or $\mathrm{W}$ bosons with $p_{\mathrm{T}} \geq 200 \mathrm{GeV}$, are usually contained within a cone of radius $R=0.8$ [86]. A collection of "large- $R$ jets", which is distinct from, and possibly overlaps with, the collection of primary jets, is used to reconstruct these boosted objects by means of the anti- $k_{\mathrm{T}}$ clustering algorithm with a distance parameter of 0.8. Additional information on jet substructure is obtained by reclustering the constituents of these jets through the Cambridge-Aachen algorithm [87]. The "modified mass drop tagger" algorithm [88], also known as the "soft drop" (SD) algorithm, with angular exponent $\beta=0$, soft cutoff threshold $z_{\text {cut }} \leq 0.1$, and characteristic radius $R_{0}=0.8$ [89], is applied to remove soft, wide-angle radiation from the jet. The performance of the SD algorithm does not depend on the algorithm used initially to reconstruct the large- $R$ jets. Top quark and $\mathrm{W}$ boson candidates are selected from the collection of large- $R$ jets after applying a loose preselection based on variables reconstructed using the SD algorithm. In our configuration, the SD algorithm identifies two hard subjets of the large- $R$ jet by reversing the Cambridge-Aachen clustering history. The two hard substructures should correspond to the $\mathrm{W}$ boson and b quark jet, in the case of top quark candidates, or to two quark jets of a $\mathrm{W}$ boson decay, in the case of a $\mathrm{W}$ boson candidate. The top quark ( $\mathrm{W}$ boson) candidates are required to have soft-drop mass $\mathrm{m}_{\mathrm{SD}} \geq 110\left(50 \leq \mathrm{m}_{\mathrm{SD}}<110\right) \mathrm{GeV}, p_{\mathrm{T}} \geq 400(200) \mathrm{GeV},|\eta| \leq 2.4$, and subjets with $p_{\mathrm{T}} \geq 20 \mathrm{GeV}$. These $\mathrm{m}_{\mathrm{SD}}$ requirements incur minimal efficiency losses, and ensure that candidates can only be tagged uniquely.

Two separate multivariate BDT are trained to identify candidates for the quark decays of highly boosted top quarks and $\mathrm{W}$ bosons. The identified objects are subsequently referred to as "merged" top quarks and W bosons, respectively. The input variables to the 

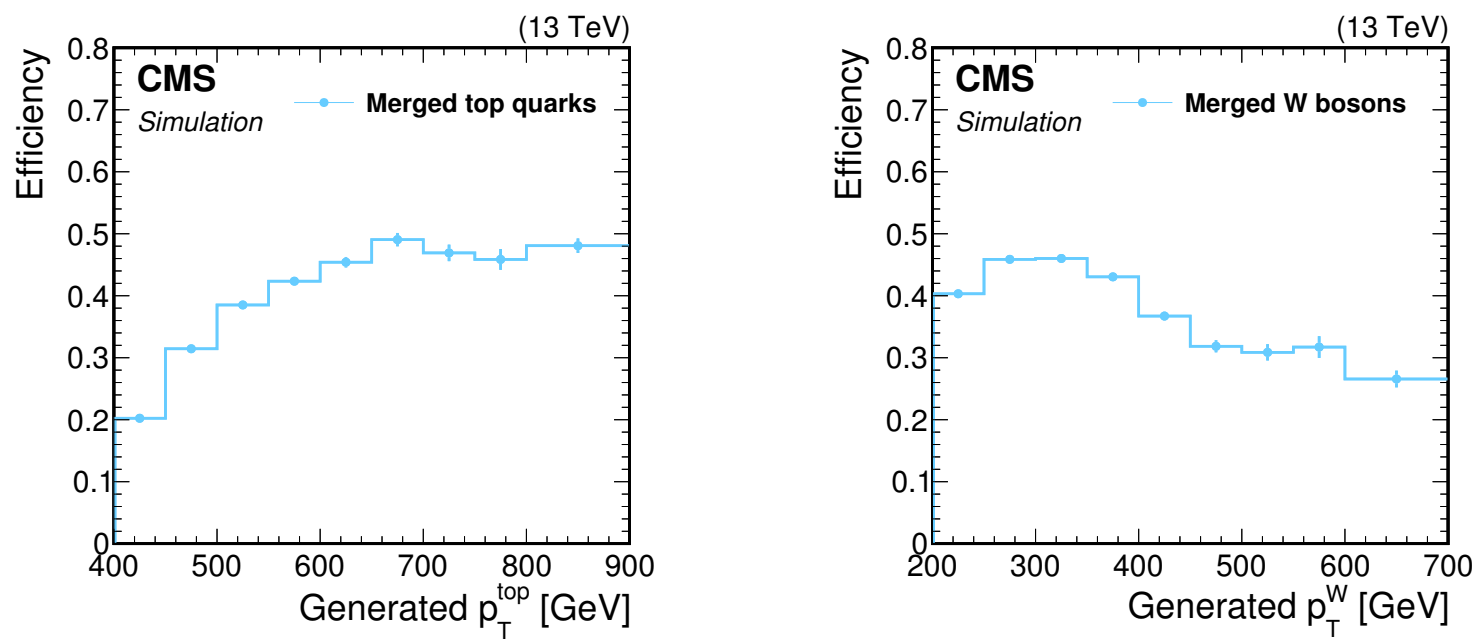

Figure 2. Efficiencies in MC simulation for identifying the quark decays of top quarks (left), and $\mathrm{W}$ bosons (right), as a function of the $p_{\mathrm{T}}$ of the generated top quarks or $\mathrm{W}$ bosons to which they were matched.

two BDT rely on $\mathrm{m}_{\mathrm{SD}}$, N-subjettiness ratios $\left(\tau_{3} / \tau_{2}\right.$ and $\left.\tau_{2} / \tau_{1}\right)$ [90], observables related to quark-gluon discrimination [91], the b tagging discriminant value, the relative difference in $p_{\mathrm{T}}$ between each of the two subjets within the large- $R$ jet, and the mass of each subjet. The $\mathrm{N}$-subjettiness variable, $\tau_{N}$, is a measure of the degree to which the jet originates from $\mathrm{N}$ subjets. The BDT are trained in MC simulated samples using the Toolkit for Multivariate Data Analysis (TMVA) [92] to discriminate between "background" and "signal" large$R$ jets. The merged top quark BDT is trained using, for "signal", candidates that are matched to generated quark decays of top quarks in simulated SUSY events. For the merged $\mathrm{W}$ boson BDT this procedure is repeated in simulated t $\overline{\mathrm{t}}$ events. For the "background" we consider the remaining candidates that could not be matched. The efficiencies to identify matched top quarks and $\mathrm{W}$ bosons are shown in figure 2. The merged $\mathrm{W}$ boson tagging efficiency is determined using $\mathrm{W}$ bosons originating from generated top quark decays; thus, the moderate drop at large $p_{\mathrm{T}}$ can be largely attributed to the merging of the top quark decay products, which reduces the effectiveness of the jet substructure variables. The misidentification rate for jets initiated by either gluons or light quarks depends on the $p_{\mathrm{T}}$ of the large- $R$ jet and ranges from 1 to $4 \%$ and from 2 to $10 \%$ for merged top quarks and $\mathrm{W}$ bosons, respectively.

The misidentification rates for these top quark and $\mathrm{W}$ boson taggers are measured in data using a sample of multijet events that is dominated by the QCD multijet process, selected with an $H_{\mathrm{T}}$ trigger, where $H_{\mathrm{T}}$ is defined as the scalar sum of the $p_{\mathrm{T}}$ of the primary jets in the event. We require the events to contain at least one large- $R$ jet and $H_{\mathrm{T}} \geq 1 \mathrm{TeV}$. The misidentification rate is measured as a function of the jet $p_{\mathrm{T}}$ and $\eta$, and then compared to the expected rates in simulation. Data-to-simulation ratios are found to deviate from unity by no more than $20 \%$, and are used to correct results obtained with simulated event samples. 
The top quark and $\mathrm{W}$ boson tagging efficiencies are measured in data using a sample of lepton+jets events dominated by the semileptonic t $\bar{t}$ process and selected using a singlemuon trigger. The muon is required to have $p_{\mathrm{T}} \geq 50 \mathrm{GeV}$ and $|\eta| \leq 2.1$. To suppress other backgrounds, at least one b-tagged jet is required in the same hemisphere as the muon, and the large- $R$ jet is required to be in the opposite hemisphere. Contributions from processes with no quark decays of top quarks or $\mathrm{W}$ bosons are corrected through misidentification correction factors applied before obtaining the tagging efficiencies. These observed efficiencies are compared to those estimated in simulation, and simulation-to-data correction factors, typically ranging from 0.9 to 1.1, are extracted and applied to simulated events to account for any dependence on $p_{\mathrm{T}}$. Simulated signal events generated in the CMS fast simulation package are corrected in a similar way for the differences in tagging performance relative to the full GEANT4-based simulations.

\subsection{Identification of intermediate- $p_{\mathrm{T}}$ top quarks}

The decay products of moderately boosted top quarks are often resolved as three separate jets in the primary jet collection. To avoid overlap with merged top quarks and $\mathrm{W}$ bosons, we only consider a "cleaned" subset of jets that are separated by a distance $R>0.8$ from all of the candidate merged objects. Three-jet "resolved" top quark candidates are formed by starting with a jet from the cleaned jet collection that is designated to be the $\mathrm{b}$ constituent jet. The two jets with highest $\mathrm{b}$ tagging discriminant values are the only eligible jets for this step. Two additional constituent jets are designated $\mathrm{W}$ constituent jets after being identified from all two-jet combinations in the cleaned collection, excluding the already designated $b$ jet. The algorithm is repeated with the remaining $b$ jet. To reduce the combinatorial background before making any stringent selections, we require the two $\mathrm{W}$ constituent jets to have invariant mass within $40 \mathrm{GeV}$ of $m_{\mathrm{W}}=80 \mathrm{GeV}$ and the combined three-jet system to have invariant mass within $80 \mathrm{GeV}$ of the top quark mass $m_{\mathrm{t}}=175 \mathrm{GeV}$. The three-jet systems that pass these requirements are considered for possible tagging as resolved top quarks.

Resolved top quark tagging is carried out using a BDT trained on simulated t $\overline{\mathrm{t}}$ events. It exploits properties of each three-jet candidate, including masses, angular separations, and other kinematic properties of the constituents. Additional input variables are quarkgluon discrimination metrics [93], b tagging discriminant values, and charm quark versus light quark jet discrimination [94] for each of the three jets. The performance of the resolved top quark tagger is shown in figure 3 . The drop in efficiency at very high $p_{\mathrm{T}}$ stems from the fact that top quark decay products are kinematically more likely to be merged into single large- $R$ jets. Correspondingly, the efficiency of the merged top quark tagger starts to become significant in this region, as seen in figure 2 (left).

The performance of the resolved top quark tagger is evaluated using the same methodology as that described in section 4.1. Simulation-to-data correction factors ranging from 1.00 to 1.15 are extracted and applied to simulated events to account for differences with data as a function of $p_{\mathrm{T}}$. Simulated signal events generated in the CMS fast simulation package are corrected in a similar way for differences in tagging performance relative to the full GEANT4-based simulations. 

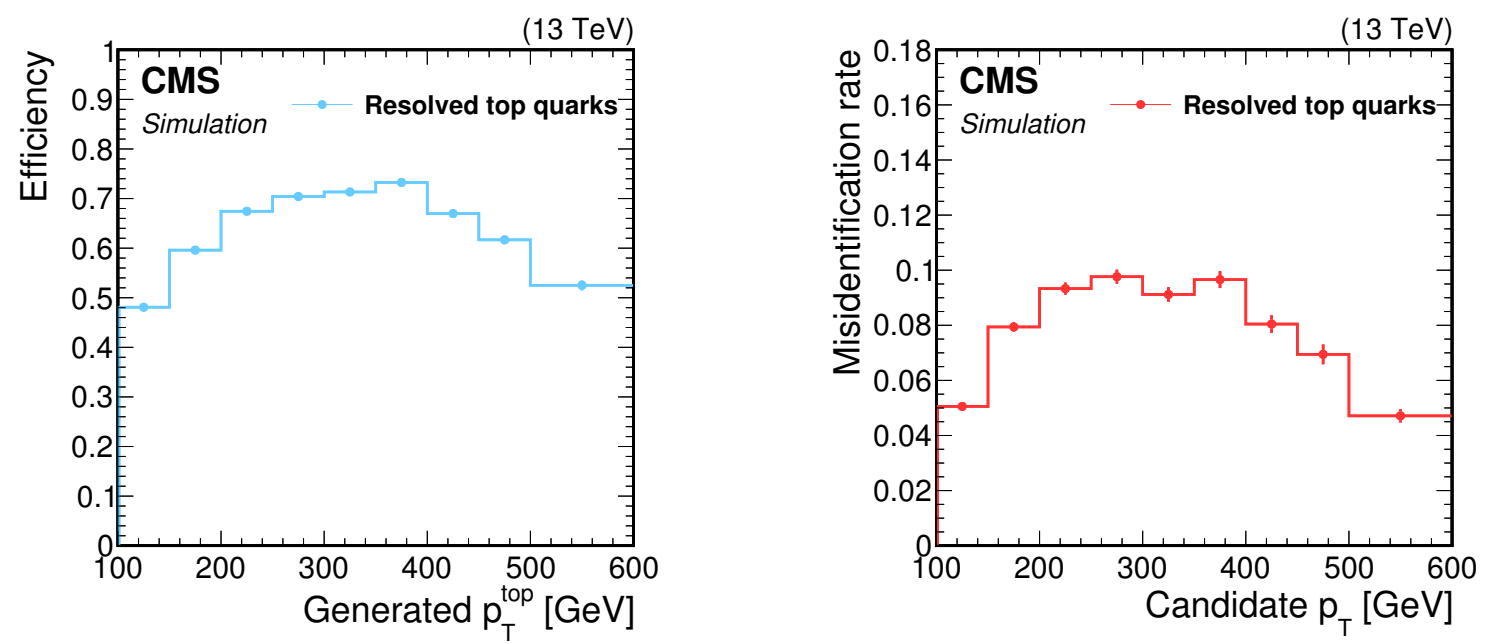

Figure 3. Left: efficiency in MC simulation to identify resolved top quark decays as a function of the $p_{\mathrm{T}}$ of the generated top quark. Right: misidentification rate in MC simulation as a function of the $p_{\mathrm{T}}$ of resolved top quarks, in a sample dominated by the QCD multijet process.

\subsection{Identification of initial-state radiation}

In models with $\Delta m<m_{\mathrm{W}}$, the LSP is much heavier than the other decay products, and the event has relatively low $p_{\mathrm{T}}^{\text {miss }}$. However, in cases where the $\widetilde{\mathrm{t}}_{1} \overline{\widetilde{\mathrm{t}}}_{1}$ pair recoils against high- $p_{\mathrm{T}}$ initial-state radiation (ISR), the massive LSP can be either moderately or highly boosted, and there can be relatively large values of $p_{\mathrm{T}}^{\text {miss }}$. To take advantage of this possibility, we try to identify an ISR jet candidate in the event. To this end, we use the set of large- $R$ jets described in section 4.1. The use of such jets improves ISR jet identification by capturing ISR gluon jets that may have undergone splitting to two or more jets that are distributed over a relatively large solid angle. For events having such jets, the large- $R$ jet with the largest value of $p_{\mathrm{T}} \geq 200 \mathrm{GeV}$ that fails the "loose" working point of the $\mathrm{b}$ tagging algorithm (characterized by a tagging efficiency of $\sim 80 \%$, and a misidentification rate of $\sim 10 \%$ for light quarks and gluons, and $\sim 40 \%$ for charm quarks) is tagged as an ISR jet candidate. This ISR jet is then used in SR that are orthogonal to those that require top quark or $\mathrm{W}$ boson candidates.

\subsection{Identification of low- $p_{\mathrm{T}} \mathrm{b}$ quarks}

As previously noted, signal models with small $\Delta m$ produce a large fraction of b quarks below the jet $p_{\mathrm{T}}$ threshold that subsequently fail to be included in the primary jet collection. Identifying these soft quarks can potentially improve our ability to separate signal events from SM background. To this end, we identify soft b hadrons, not associated to jets, by means of a secondary vertex (SV) reconstructed by the inclusive vertex finding algorithm [95]. Additional requirements for SV observables are used to suppress background from light-flavor hadrons and jets. These include the distance in the transverse plane between the SV and PV; the significance of this distance; its pointing angle, defined through the scalar product between the distance vector and the $\vec{p}_{\mathrm{SV}}$ direction as $\cos \left(\overrightarrow{(\mathrm{PV}, \mathrm{SV})}, \vec{p}_{\mathrm{SV}}\right)$, 
where $\vec{p}_{\mathrm{SV}}$ is the total momentum of the tracks associated with the SV; and the number of tracks associated with the SV. The transverse momenta of the tracks associated with an SV are required to sum to $p_{\mathrm{T}}<20 \mathrm{GeV}$, and be separated from any jets (including b-tagged jets) by $R>0.4$. This definition leads to $\sim 20 \%$ efficiency to identify a b hadron in the $p_{\mathrm{T}}$ range from 10 to $20 \mathrm{GeV}$, for a misidentification rate less than one percent. The soft $\mathrm{b}$ tagging efficiency in data is measured in a sample dominated by $\mathrm{t} \overline{\mathrm{t}}$ events having an $\mathrm{e} \mu$ pair, $p_{\mathrm{T}}^{\text {miss }} \geq 50 \mathrm{GeV}$, a b-tagged jet, and no additional jets. The presence of an additional soft $\left(p_{\mathrm{T}}<20 \mathrm{GeV}\right)$, nonisolated $\mu$ is used to estimate the fraction of soft b quarks in data. The soft $\mathrm{b}$ tagging performance in simulation agrees with the performance in data within $16 \%$. Simulated signal events produced in the CMS fast simulation package are corrected for differences in soft b tagging relative to GEANT4-based simulations.

\section{$5 \quad$ Search strategy}

With the final-state signatures of the signals in mind, we select events collected with a $p_{\mathrm{T}}^{\text {miss }}$ trigger and require $p_{\mathrm{T}}^{\text {miss }} \geq 250 \mathrm{GeV}$ offline. The SM backgrounds with intrinsic $p_{\mathrm{T}}^{\text {miss }}$ generated through the leptonic decay of a $\mathrm{W}$ boson are significantly suppressed by rejecting events containing isolated electrons or muons with $p_{\mathrm{T}} \geq 5 \mathrm{GeV},|\eta| \leq 2.4$, and $I_{\text {rel }} \leq 0.1$, or $I_{\text {rel }} \leq 0.2$, respectively. The contribution from events in which a $\mathrm{W}$ boson decays to a $\tau$ lepton is suppressed by rejecting events containing isolated $\tau_{\mathrm{h}}$ candidates.

In our "search sample", defined by the above requirements, the dominant sources of SM background with intrinsic $p_{\mathrm{T}}^{\text {miss }}$ are $\mathrm{t} \overline{\mathrm{t}}, \mathrm{W}+$ jets, and $\mathrm{Z}+$ jets, single top quark, and $\mathrm{t} \overline{\mathrm{ZZ}}$ processes. The contribution from $\mathrm{t} \overline{\mathrm{t}}$ and $\mathrm{W}+$ jets processes arises from events in which $\mathrm{W}$ bosons decay leptonically to produce $p_{\mathrm{T}}^{\text {miss }}$ associated with an energetic neutrino, but the charged lepton either falls outside of the kinematic acceptance, or, even more likely, may be misidentified as a jet after failing to be identified as a lepton. This background is collectively

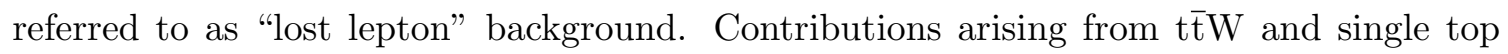
quark processes also enter this category at lower levels. The contributions from $\mathrm{Z}+$ jets and $t \bar{t} Z$ events arise when the $\mathrm{Z}$ boson decays to neutrinos, thereby producing significant $p_{\mathrm{T}}^{\text {miss }}$. Contributions from the QCD multijet process enter the search sample in cases where severe mismeasurements of jet momenta (i.e., jets passing through dead regions, cracks, or transition regions of the detector) produce significant artificial $p_{\mathrm{T}}^{\text {miss }}$, or when neutrinos arise from leptonic decays of heavy-flavor hadrons produced during jet fragmentation.

We define a total of 104 non-overlapping SR with two sets of disjoint baseline selection criteria that are designed specifically for application in the high and low $\Delta m$ signals. Tables 1 and 2 summarize these criteria for the 51 high $\Delta m$ SR and 53 low $\Delta m$ SR, respectively.

\subsection{Strategy for high $\Delta m$ models}

Based on the final-state signatures of models with $\Delta m>m_{\mathrm{W}}$, we define a high $\Delta m$ baseline selection that requires at least five jets in our primary jet collection $\left(N_{\mathrm{j}} \geq 5\right)$, of which at least one is b-tagged $\left(N_{\mathrm{b}} \geq 1\right)$. Severely mismeasured high- $p_{\mathrm{T}}$ jets in multijet events can lead to large values of $p_{\mathrm{T}}^{\text {miss }}$ but generally have $\vec{p}_{\mathrm{T}}^{\text {miss }}$ aligned with one of the higher- $p_{\mathrm{T}}$ jets 
in the event. We therefore add the requirement of separation in azimuthal angle between $\vec{p}_{\mathrm{T}}^{\text {miss }}$ and each of the four jets with largest $p_{\mathrm{T}}, \Delta \phi_{1234} \geq 0.5$, which greatly reduces the contribution from this background. Events passing the high $\Delta m$ baseline selection are then divided into multiple non-overlapping SR, optimized for the kinematic properties of moderate to high $\Delta m$ signal topologies.

In lepton + jets $t \bar{t}$ events, where most of the $p_{T}^{\text {miss }}$ is due to the leptonic decay of a single $\mathrm{W}$ boson, the transverse mass distribution of the neutrino and b quark from the same top quark decay has an endpoint at the mass of the top quark. To take advantage of this fact, we separate events based on the value of the smallest b quark transverse mass in the event, $m_{\mathrm{T}}^{\mathrm{b}}($ see $(2))$. In case there are more than two b-tagged jets, only the two jets with the highest $b$ tagging discriminant value are considered. The two resulting sets of events are the $\mathrm{t} \overline{\mathrm{t}}$-depleted high- $m_{\mathrm{T}}^{\mathrm{b}}$ category (with $m_{\mathrm{T}}^{\mathrm{b}} \geq 175 \mathrm{GeV}$ ), and the $\mathrm{t} \overline{\mathrm{t}}$-enhanced low- $m_{\mathrm{T}}^{\mathrm{b}}$ category (with $m_{\mathrm{T}}^{\mathrm{b}}<175 \mathrm{GeV}$ ).

To target signals with moderate values of $\Delta m$ that populate the low- $m_{\mathrm{T}}^{\mathrm{b}}$ category, we require the presence of at least one resolved top quark and $N_{\mathrm{j}} \geq 7$. The latter condition assures that a signal event would contain at least one radiated jet, providing a boost to the system and thereby increasing the $p_{\mathrm{T}}^{\text {miss }}$ for better discrimination from backgrounds. The high- $m_{\mathrm{T}}^{\mathrm{b}}$ category is subdivided into two categories: events that do not contain any top quark or $\mathrm{W}$ boson candidates with the requirement $N_{\mathrm{j}} \geq 7$, and events that do contain top quark or $\mathrm{W}$ boson candidates, as expected for models with larger values of $\Delta m$ and highly boosted top quarks or $\mathrm{W}$ bosons. In the latter case, we retain the baseline requirement of $N_{\mathrm{j}} \geq 5$ and define separate $\mathrm{SR}$ according to the numbers of candidate merged top quarks $\left(N_{\mathrm{t}}\right)$, merged $\mathrm{W}$ bosons $\left(N_{\mathrm{W}}\right)$, and resolved top quarks $\left(N_{\text {res }}\right)$. All these regions are further subdivided into SR according to the number of b-tagged jets, $N_{\mathrm{b}}=1$ or $\geq 2$, and different ranges of $p_{\mathrm{T}}^{\mathrm{miss}}$.

\subsection{Strategy for low $\Delta m$ models}

The low $\Delta m$ baseline selection is most appropriate for models with $\Delta m<m_{\mathrm{W}}$. To this end, we select events that have at least two jets, no top quark or $\mathrm{W}$ boson candidates, and small $m_{\mathrm{T}}^{\mathrm{b}}(<175 \mathrm{GeV})$ when there are b-tagged jets present. In addition, we require an ISR jet with $p_{\mathrm{T}}^{\mathrm{ISR}} \geq 300 \mathrm{GeV},|\eta| \leq 2.4$, and $\left|\Delta \phi\left(j_{\mathrm{ISR}}, \vec{p}_{\mathrm{T}}^{\text {miss }}\right)\right| \geq 2$, where the last requirement suppresses the QCD multijet process. As discussed in section 4.3, the requirement of an ISR jet provides sensitivity to low $\Delta m$ signal topologies, in which intrinsic $p_{\mathrm{T}}^{\mathrm{miss}}$ is generated by the decay of $\widetilde{\mathrm{t}}_{1} \overline{\widetilde{\mathrm{t}}}_{1}$ pairs recoiling against ISR. To further suppress the QCD multijet process, we require $\left|\Delta \phi\left(\mathrm{j}_{1}, \vec{p}_{\mathrm{T}}^{\text {miss }}\right)\right| \geq 0.5,\left|\Delta \phi\left(\mathrm{j}_{2,3}, \vec{p}_{\mathrm{T}}^{\text {miss }}\right)\right| \geq 0.15$, where $\mathrm{j}_{1}, \mathrm{j}_{2}, \mathrm{j}_{3}$ are the three leading$p_{\mathrm{T}}$ jets. In addition, a measure of significance in $p_{\mathrm{T}}^{\text {miss }}$, defined as $p_{\mathrm{T}}^{\text {miss }} / \sqrt{H_{\mathrm{T}}} \equiv S_{E_{\mathrm{T}}} \geq$ $10 \sqrt{\mathrm{GeV}}$, is required to ensure that $p_{\mathrm{T}}^{\text {miss }}$ can only arise from undetectable particles or very rare, extreme mismeasurements.

Events satisfying the above requirements are further subdivided into SR defined by $N_{\mathrm{b}}$, the number of identified secondary vertices $N_{\mathrm{SV}}, p_{\mathrm{T}}^{\mathrm{ISR}}$, and $p_{\mathrm{T}}^{\text {miss }}$. Events with $N_{\mathrm{b}}=0$, a category used for very soft decay products, are further subdivided by ranges of $N_{\mathrm{j}}, 2$ to 5 or $\geq 6, N_{\mathrm{SV}}$, and $p_{\mathrm{T}}^{\text {miss }}$, after requiring very high $p_{\mathrm{T}}^{\mathrm{ISR}}$ to assure a substantial boost to finalstate jets which, in turn, enhances the effectiveness of soft $\mathrm{b}$ tagging by producing more 


\begin{tabular}{|c|c|c|c|c|c|}
\hline \multicolumn{6}{|c|}{$m_{\mathrm{T}}^{\mathrm{b}}<175 \mathrm{GeV}$} \\
\hline$N_{\mathrm{j}}$ & $N_{\mathrm{t}}$ & $N_{\mathrm{W}}$ & $N_{\text {res }}$ & $N_{\mathrm{b}}$ & $p_{\mathrm{T}}^{\mathrm{miss}}[\mathrm{GeV}]$ \\
\hline$\geq 7$ & $\geq 0$ & $\geq 0$ & $\geq 1$ & $1, \geq 2$ & $250-300,300-400,400-500, \geq 500$ \\
\hline \multicolumn{6}{|c|}{$m_{\mathrm{T}}^{\mathrm{b}} \geq 175 \mathrm{GeV}$} \\
\hline$N_{\mathrm{j}}$ & $N_{\mathrm{t}}$ & $N_{\mathrm{W}}$ & $N_{\text {res }}$ & $N_{\mathrm{b}}$ & $p_{\mathrm{T}}^{\mathrm{miss}}[\mathrm{GeV}]$ \\
\hline$\geq 7$ & 0 & 0 & 0 & $1, \geq 2$ & $250-350,350-450,450-550, \geq 550$ \\
\hline \multirow{4}{*}{$\geq 5$} & $\geq 1$ & 0 & 0 & \multirow{4}{*}{1} & $550-650, \geq 650$ \\
\hline & 0 & 0 & $\geq 1$ & & $250-350,350-450,450-550,550-650, \geq 650$ \\
\hline & $\geq 1$ & $\geq 1$ & 0 & & $\geq 550$ \\
\hline & 0 & $\geq 1$ & $\geq 1$ & & $250-350,350-450,450-550, \geq 550$ \\
\hline \multirow{9}{*}{$\geq 5$} & 1 & 0 & 0 & \multirow{9}{*}{$\geq 2$} & $550-650, \geq 650$ \\
\hline & 0 & 1 & 0 & & $250-350,350-450,450-550,550-650, \geq 650$ \\
\hline & 0 & 0 & 1 & & $250-350,350-450,450-550,550-650, \geq 650$ \\
\hline & 1 & 1 & 0 & & $\geq 550$ \\
\hline & 0 & 1 & 1 & & $250-350,350-450,450-550, \geq 550$ \\
\hline & 1 & 0 & 1 & & $250-350,350-450, \geq 450$ \\
\hline & $\geq 2$ & 0 & 0 & & $\geq 250$ \\
\hline & 0 & $\geq 2$ & 0 & & $\geq 250$ \\
\hline & 0 & 0 & $\geq 2$ & & $\geq 250$ \\
\hline
\end{tabular}

Table 1. Summary of the 51 non-overlapping search regions that mainly target high $\Delta m$ signal. The high $\Delta m$ baseline selection is $N_{\mathrm{j}} \geq 5, p_{\mathrm{T}}^{\text {miss }} \geq 250 \mathrm{GeV}$, no leptons, $N_{\mathrm{b}} \geq 1$, and $\Delta \phi_{1234} \geq 0.5$.

significantly displaced b hadron decays. The $\mathrm{SR}$ with $N_{\mathrm{b}}=N_{\mathrm{SV}}=0$ provide sensitivity to the T2cc model. They may also provide sensitivity to similar final states involving lighter quarks but we have not studied these cases. Events with $N_{\mathrm{b}} \geq 1$ are subdivided according to the scalar sum of the $p_{\mathrm{T}}$ of the leading and subleading (if one is present) b-tagged jets, $p_{\mathrm{T}}^{\mathrm{b}}$, to take advantage of the softer $\mathrm{b}$ jet $p_{\mathrm{T}}$ spectrum expected from the low $\Delta m$ models relative to the SM background.

\section{Background estimation}

The contribution of each SM background process to the search sample is estimated through measurements of dedicated control data events that are translated to predictions for event counts in the corresponding SR with the aid of simulation. The strategy makes use of methods described in ref. [44]. 


\begin{tabular}{|cccccc|}
\hline$N_{\mathrm{j}}$ & $N_{\mathrm{b}}$ & $N_{\mathrm{SV}}$ & $p_{\mathrm{T}}^{\mathrm{ISR}}[\mathrm{GeV}]$ & $p_{\mathrm{T}}^{\mathrm{b}}[\mathrm{GeV}]$ & $p_{\mathrm{T}}^{\text {miss }}[\mathrm{GeV}]$ \\
\hline $2-5$ & & 0 & & & $450-550,550-650,650-750, \geq 750$ \\
$\geq 6$ & & 0 & & & $450-550,550-650,650-750, \geq 750$ \\
$2-5$ & 0 & $\geq 1$ & $\geq 500$ & - & $450-550,550-650,650-750, \geq 750$ \\
$\geq 6$ & & $\geq 1$ & & & $450-550,550-650,650-750, \geq 750$ \\
\hline & & 0 & $300-500$ & $20-40$ & $300-400,400-500,500-600, \geq 600$ \\
& & 0 & $300-500$ & $40-70$ & $300-400,400-500,500-600, \geq 600$ \\
$\geq 2$ & 1 & 0 & $\geq 500$ & $20-40$ & $450-550,550-650,650-750, \geq 750$ \\
& & 0 & $\geq 500$ & $40-70$ & $450-550,550-650,650-750, \geq 750$ \\
& & $\geq 1$ & $\geq 300$ & $20-40$ & $300-400,400-500, \geq 500$ \\
$\geq 2$ & & & $300-500$ & $40-80$ & $300-400,400-500, \geq 500$ \\
$\geq 2$ & & & $300-500$ & $80-140$ & $300-400,400-500, \geq 500$ \\
$\geq 7$ & & & $300-500$ & $\geq 140$ & $300-400,400-500, \geq 500$ \\
$\geq 2$ & $\geq 2$ & $\geq 0$ & $\geq 500$ & $40-80$ & $450-550,550-650, \geq 650$ \\
$\geq 2$ & & & $\geq 500$ & $80-140$ & $450-550,550-650, \geq 650$ \\
$\geq 7$ & & & $\geq 300$ & $\geq 140$ & $450-550,550-650, \geq 650$ \\
\hline
\end{tabular}

Table 2. Summary of the 53 non-overlapping search regions that mainly target low $\Delta m$ signal. The low $\Delta m$ baseline selection is $N_{\mathrm{j}} \geq 2, p_{\mathrm{T}}^{\text {miss }} \geq 250 \mathrm{GeV}$, no leptons, $N_{\mathrm{t}}=N_{\mathrm{W}}=N_{\text {res }}=0$, $m_{\mathrm{T}}^{\mathrm{b}}<175 \mathrm{GeV}$ (when applicable), $\left|\Delta \phi\left(\mathrm{j}_{1}, \vec{p}_{\mathrm{T}}^{\text {miss }}\right)\right| \geq 0.5, \quad\left|\Delta \phi\left(\mathrm{j}_{2,3}, \vec{p}_{\mathrm{T}}^{\text {miss }}\right)\right| \geq 0.15$, and an ISR jet with $p_{\mathrm{T}}^{\mathrm{ISR}} \geq 300 \mathrm{GeV},|\eta| \leq 2.4,\left|\Delta \phi\left(j_{\mathrm{ISR}}, \vec{p}_{\mathrm{T}}^{\text {miss }}\right)\right| \geq 2$, and $S_{E_{\mathrm{T}}} \geq 10 \sqrt{\mathrm{GeV}}$.

\subsection{Estimation of the lost-lepton background}

The lost-lepton (LL) background is estimated from a single-lepton control sample that is based on a sample of events collected with the same $p_{\mathrm{T}}^{\text {miss }}$ trigger as the search sample. We create a relatively pure single lepton sample (" 1 ") by inverting the electron or muon veto requirements described in section 5 . More than $90 \%$ of the events in these samples contain a single lepton, while the remainder contain two or more leptons. Studies of simulated events indicate that event kinematic variables for different lepton flavors are sufficiently similar to provide a collective estimate of LL backgrounds from a single control sample. Potential contamination by signal is suppressed by requiring $m_{\mathrm{T}}\left(\vec{p}_{\mathrm{T}}(\ell), \vec{p}_{\mathrm{T}}^{\mathrm{miss}}\right)<100 \mathrm{GeV}$, consistent with the expectation for a $\mathrm{W}$ boson decay. In events with more than one identified lepton, the one used in this calculation is selected randomly. The selection criteria applied to the single-lepton control sample are the same as those used in the search sample, with the exception of top quark and $\mathrm{W}$ boson multiplicity, as discussed below.

The LL estimation in each SR is based upon the event count in corresponding singlelepton control regions (CR). The count is translated to a prediction in the SR by means of a transfer factor obtained from simulation, as follows:

$$
N_{\text {pred }}^{\mathrm{LL}}=T F_{\mathrm{LL}} N_{\text {data }}(1 \ell)
$$


where $N_{\text {data }}(1 \ell)$ corresponds to the event count observed in the relevant single-lepton CR in data, and the transfer factor, $T F_{\mathrm{LL}}$, translates $N_{\text {data }}(1 \ell)$ to a background prediction in the $\mathrm{SR}, N_{\text {pred }}^{\mathrm{LL}}$, and is defined as:

$$
T F_{\mathrm{LL}}=\frac{N_{\mathrm{MC}}(0 \ell)}{N_{\mathrm{MC}}(1 \ell)},
$$

where $N_{\mathrm{MC}}(0 \ell)$ and $N_{\mathrm{MC}}(1 \ell)$ are the LL yields found for simulated events in the search and single-lepton samples, respectively, that include contributions from t $\overline{\mathrm{t}}$ and $\mathrm{W}+$ jets events, as well as smaller contributions from single top quark and t $\bar{t} \mathrm{~W}$ processes.

To improve the statistical uncertainty of this background estimation, CR relevant to the high $\Delta m$ SR are combined for all top quark and $\mathrm{W}$ boson multiplicities in both data and simulation. The top quark and $\mathrm{W}$ boson tagger results for the simulated events are corrected by the simulation-to-data correction factors discussed in section 4 . Simulation is used to extrapolate these results to each SR with its particular top quark and W boson multiplicity. The selection efficiency for each of the other search variables is estimated directly from data in the single-lepton sample.

\subsection{Estimation of the $\mathrm{Z}(\nu \nu)$ background}

An important source of background in the search arises from events in which a $\mathrm{Z}$ boson, produced in association with jets, decays to neutrinos that carry away large $p_{\mathrm{T}}^{\text {miss }}$. Two methods are traditionally used $[39,41]$ to estimate this background. The first method uses an event sample dominated by $\mathrm{Z}(\ell \ell)+$ jets events, in which the $\mathrm{Z}$ bosons have kinematic properties very similar to those in the search sample, after correcting for the difference in acceptance between charged lepton pairs and pairs of neutrinos. One drawback in this is that these events are statistically limited, especially in the stringently defined SR often used in SUSY searches. To overcome this limitation, the second method utilizes $\gamma+$ jets events, in which the $\gamma+$ jets process has similar LO Feynman diagrams to the $\mathrm{Z}+$ jets process, but is more copious than the $\mathrm{Z}(\ell \ell)+$ jets by about a factor of five. To use this sample requires taking into account the differences in quark-boson couplings and the fact that the $\mathrm{Z}$ boson is very massive. Fortunately, these differences are substantially reduced for the high- $p_{\mathrm{T}}$ bosons in this search.

Considering the pros and cons of the two methods led us to use a hybrid method to estimate the $\mathrm{Z}(\nu \nu)$ background that makes use of both procedures. We use the $\mathrm{Z}(\ell \ell)+$ jets sample to get the normalization of the $\mathrm{Z}(\nu \nu)+$ jets background. This is done in different ranges of $N_{\mathrm{b}}$ and $N_{\mathrm{SV}}$ to account for dependence on heavy-flavor production. Meanwhile, the $\gamma+$ jets events are used to correct the $p_{\mathrm{T}}^{\text {miss }}$ distributions of simulated events. The $\mathrm{Z}(\ell \ell)$ sample is collected with dielectron and dimuon triggers that require the leading electron (muon) to have $p_{\mathrm{T}} \geq 25(20) \mathrm{GeV}$, and the subleading electron (muon) to have $p_{\mathrm{T}} \geq 15(10) \mathrm{GeV}$. The leptons must have $|\eta| \leq 2.4$ to be within the acceptance of the tracker. The $\gamma+$ jets events are collected with a single-photon trigger and an offline selection of $p_{\mathrm{T}} \geq 200 \mathrm{GeV}$ and $|\eta| \leq 2.5$ for the leading photon. To suppress potential contributions from signals and to avoid overlap with the search sample we consider only the events with $p_{\mathrm{T}}^{\text {miss }}<200 \mathrm{GeV}$. The transverse momentum of the boson, as determined from the lepton 
pair or the photon, is added vectorially to $\vec{p}_{\mathrm{T}}^{\text {miss }}$ to emulate the kinematic properties of the $\mathrm{Z}(\nu \nu)+$ jets process. The modified $p_{\mathrm{T}}^{\text {miss }}$, denoted by $p_{\mathrm{T}}^{\text {miss, } \ell \ell}$ and $p_{\mathrm{T}}^{\text {miss, } \gamma}$ for the $\mathrm{Z}(\ell \ell)+$ jets and $\gamma+$ jets processes, respectively, is used to calculate the relevant kinematic variables.

The prediction for the $\mathrm{Z}(\nu \nu)$ background in any particular SR is given by:

$$
N_{\mathrm{Z} \rightarrow \nu \nu}^{\mathrm{pred}}=N_{\mathrm{Z} \rightarrow \nu \nu}^{\mathrm{sim}} R_{\mathrm{Z}} S_{\gamma}
$$

where $N_{\mathrm{Z} \rightarrow \nu \nu}^{\operatorname{sim}}$ is the expected number of $\mathrm{Z}(\nu \nu)$ events in simulation, $R_{\mathrm{Z}}$ is the flavordependent $\mathrm{Z}+$ jets normalization factor measured using the $\mathrm{Z}(\ell \ell)$ events, and $S_{\gamma}$ is the correction factor for the $p_{\mathrm{T}}^{\text {miss }}$ distribution as extracted from the $\gamma+$ jets events in data.

The factor $R_{\mathrm{Z}}$ is calculated by comparing the observed and expected $\mathrm{Z}(\ell \ell)$ yields after applying the baseline selection criteria, with the exception of the requirements on the azimuthal angles between jets and $p_{\mathrm{T}}^{\text {miss }}$. The latter are omitted to retain more events and hence reduce the statistical uncertainty in the $R_{Z}$ estimation, after first confirming that this omission does not bias the result. To increase the purity of the $\mathrm{Z}(\ell \ell)$ sample, we require the dilepton invariant mass to lie within the $\mathrm{Z}$ boson mass window of $80 \leq M_{\ell \ell}<100 \mathrm{GeV}$. To probe similar phase space as in the search sample, the $p_{\mathrm{T}}$ of the dilepton system is required to be above $200 \mathrm{GeV}$. The normalization of the nonnegligible $t \bar{t}$ contamination is estimated from the sidebands of the $\mathrm{Z}$ boson mass window of $50 \leq M_{\ell \ell}<80$ and $M_{\ell \ell} \geq 100 \mathrm{GeV}$. Small contributions from $\mathrm{tZ}, \mathrm{t} \overline{\mathrm{t} Z}, \mathrm{WZ}$, and ZZ production, estimated from simulation, are included in the $\mathrm{Z}(\ell \ell)$ sample when measuring $R_{\mathrm{Z}}$; whereas contributions from $\mathrm{tW}$, $\mathrm{t} \overline{\mathrm{t}} \mathrm{W}$, and $\mathrm{WW}$ are included in the simulated sample used to obtain the normalization factor for the $t \bar{t}$ contamination. To account for effects related to heavy-flavor production, $R_{\mathrm{Z}}$ is calculated separately for the $N_{\mathrm{b}}$ and $N_{\mathrm{SV}}$ requirements used in different SR. The $R_{\mathrm{Z}}$ values are consistent with unity. The uncertainty in $R_{Z}$, ranging from 1 to $29 \%$, comes mainly from the event counts in data and simulation after implementing the selections, and is treated as a systematic uncertainty in the prediction of the $\mathrm{Z}(\nu \nu)$ background.

The correction factor $S_{\gamma}$ is calculated in each of the search categories via a comparison of the $p_{\mathrm{T}}^{\text {miss }, \gamma}$ distributions of $\gamma+$ jets events in simulation and data. The event count from simulation is first normalized to the number of events in data after applying the appropriate $\Delta m$ baseline selections. The $S_{\gamma}$ factor is estimated separately for each SR, to account for any potential mismodeling of the search variables in simulation. As for the LL background estimation, good agreement between simulation and data for the performance of the top quark and $\mathrm{W}$ boson taggers provides a way for us to combine CR for all multiplicities of top quarks and W bosons to calculate $S_{\gamma}$, thereby improving the statistical uncertainty of the result. We then use simulation to extrapolate these results to each SR with its particular top quark and $\mathrm{W}$ boson multiplicity, after correcting events using the simulation-to-data correction factors discussed in section 4 .

An underlying assumption of the hybrid estimation is that any differences between data and simulation in the $p_{\mathrm{T}}^{\text {miss }}$ distributions for $\mathrm{Z}(\nu \nu)$ events should be compatible with those in the $p_{\mathrm{T}}^{\text {miss, } \gamma}$ distributions for photon events. We checked this assumption by comparing the ratios of data to simulation for the $p_{\mathrm{T}}^{\text {miss }, \ell \ell}$ and $p_{\mathrm{T}}^{\text {miss, } \gamma}$ distributions of $\mathrm{Z}(\ell \ell)+$ jets and $\gamma+$ jets samples, respectively. Residual differences in data and simulation can arise in the process of 
object reconstruction or as a result of the absence of higher-order corrections in simulation. Observed differences are included in the systematic uncertainties of the $\mathrm{Z}(\nu \nu)$ prediction.

\subsection{Estimation of the QCD multijet background}

The background originating from the QCD multijet process generally constitutes less than $10 \%$ of the total SM background in the SR. It is estimated using a control region in data, consisting of events collected with the same trigger as that used in the search. A sample dominated by the QCD multijet process is then obtained by requiring the azimuthal angle between any one of the three leading jets and $p_{\mathrm{T}}^{\text {miss }}, \Delta \phi_{123}$, to be smaller than 0.1 .

We again translate the observation in the control sample to a prediction in the search sample by means of transfer factors obtained from simulation. Each transfer factor is defined by the ratio between the number of simulated QCD multijet events satisfying the SR selection on the azimuthal angles of the four leading jets and $p_{\mathrm{T}}^{\text {miss }}$, to the number of simulated QCD multijet events satisfying $\Delta \phi_{123} \leq 0.1$. Contributions from other SM processes to the QCD multijet control sample are subtracted after normalizing the simulation to data in dedicated control samples. The estimation is made in each SR. To improve the statistical uncertainty of the prediction, we combine the CR over $N_{\mathrm{t}}, N_{\mathrm{W}}$, and $N_{\text {res }}$, in data and in simulation. In similarity with the estimations of the LL and $\mathrm{Z}(\nu \nu)$ backgrounds, we extrapolate in top quark and $\mathrm{W}$ boson multiplicity using simulation that is corrected for differences in the top quark and $\mathrm{W}$ boson tagging performance with respect to data. In the low $\Delta m$ SR categories, we also combine regions of $p_{\mathrm{T}}^{\text {miss }}$ in the QCD multijet control sample when yields are limited for the CR defined by $N_{\mathrm{b}} \geq 1$, and we assign an uncertainty for the combination based on the data-to-simulation ratios observed in CR with $N_{\mathrm{b}}=0$.

The dominant source of events originating from QCD multijet processes that populate the $\mathrm{SR}$ is from the severe mismeasurement of the $p_{\mathrm{T}}$ of one or more jets in the event, which translates to large values of artificial $p_{\mathrm{T}}^{\text {miss }}$. The level of mismeasurement can be parameterized via the response variable $r_{\text {jet }}$, defined as the ratio of the reconstructed $p_{\mathrm{T}}$ of the most mismeasured jet in the event to its generated $p_{\mathrm{T}}$, computed without including the loss of visible momentum due to neutrinos. The most mismeasured jet is selected based on the jet with greatest absolute difference between the reconstructed and generated $p_{\mathrm{T}}$. In data, we construct the observable $r_{\text {jet }}^{\text {pseudo }}$, defined as the ratio of the $p_{\mathrm{T}}$ of a given jet to the magnitude of the vector sum of $\vec{p}_{\mathrm{T}}$ and the total $\vec{p}_{\mathrm{T}}^{\text {miss }}$ of the event, which offers a measure of the true jet response. The jet closest in $\phi$ to $\vec{p}_{\mathrm{T}}^{\text {miss }}$ is chosen for this calculation. Mismeasurement correction factors are extracted by comparing the $r_{\text {jet }}^{\text {pseudo }}$ distributions in data and simulation. The correction factors are parameterized as functions of $r_{\text {jet }}$ and flavor of the most mismeasured jet. The corrections range from 4 to $82 \%$, and are applied to the simulation on an event-by-event basis.

Due to the large production cross section of the QCD multijet process, samples of simulated QCD multijet events entering the stringently defined SR have limited statistics. To increase it, we use a method that lets each event from the original sample appear multiple times. To this end, we use event "smearing", whereby a "new" event is created by randomly assigning $r_{\text {jet }}$ values to the leading two jets, ranked by their generated jet $p_{\mathrm{T}}$, and then recalculating all search variables based on the "smeared" jets. The $r_{\text {jet }}$ values are 
sampled from inclusive $r_{\text {jet }}$ distributions binned in both generated jet $p_{\mathrm{T}}$ and jet flavor in a region centered on the original $r_{\text {jet }}$ value. Each original event is smeared 100 times, and the statistical uncertainty in evaluated quantities is estimated through a bootstrapping procedure [96] that utilizes 50 pseudo-experiments. We assign a systematic uncertainty of $50 \%$ based on the measured difference in the distribution of the azimuthal angles between the leading jets and $p_{\mathrm{T}}^{\text {miss }}$ before and after smearing. This accounts for any potential bias introduced in this method.

\subsection{Estimation of "rare" SM backgrounds}

Contributions from diboson (WW, WZ, and ZZ) processes are relatively small compared to the other backgrounds discussed above, and mainly affect the SR in the low $\Delta m$ analysis. We therefore estimate this background directly from simulation, with an uncertainty in the production cross section of 50\% [97-99]. The t昏 contribution is also generally very small due to the rarity of this process. However, in SR requiring more than one top quark or $\mathrm{W}$ boson, this process can constitute a significant fraction of the total background due

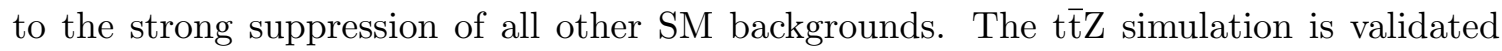
using a three-lepton control sample, obtained using single-lepton triggers, requiring the presence of exactly three leptons (electrons or muons) that satisfy $p_{\mathrm{T}} \geq 40 \mathrm{GeV}$ for the leading lepton, $p_{\mathrm{T}} \geq 20 \mathrm{GeV}$ for the second and third lepton, and no additional lepton with $p_{\mathrm{T}} \geq 10 \mathrm{GeV}$. We further require at least five jets, of which at least two are b-tagged. The same-flavor, opposite-sign lepton pair with the highest dilepton $p_{\mathrm{T}}$ is assumed to originate from $\mathrm{Z}$ boson decay. We require the presence of such a pair with the invariant mass near the $\mathrm{Z}$ boson mass $(80-100 \mathrm{GeV})$ and $p_{\mathrm{T}}$ greater than $100 \mathrm{GeV}$ to probe boson kinematic properties similar to those in the search sample. The region outside the $\mathrm{Z}$ boson mass window is used to constrain the $t \bar{t}$ background. We find that yields in simulated $t \bar{t} Z$ agree with those observed in data. An uncertainty of $24 \%$ is assigned to the normalization of the t⿱亠幺ZZ background in the SR, based on the statistical uncertainty of the simulation-todata correction factor obtained from this comparison. To assess any potential bias related to the extrapolation from the $\mathrm{Z}$ boson $p_{\mathrm{T}}\left(p_{\mathrm{T}}(\mathrm{Z})\right)$ range of the control sample to that of the search sample, we evaluate the $t \bar{t} Z$ simulation-to-data correction factors with different requirements on the reconstructed $p_{\mathrm{T}}(\mathrm{Z})$, and find the $p_{\mathrm{T}}$-binned correction factors to be consistent with the inclusive correction factor evaluated for $p_{\mathrm{T}} \geq 100 \mathrm{GeV}$. Theoretical uncertainties related to the choice of PDF and renormalization $\left(\mu_{\mathrm{R}}\right)$ and factorization $\left(\mu_{\mathrm{F}}\right)$ scales are found to be up to $28 \%$ in simulated events.

\subsection{Validation of the background methods in data}

The background estimation strategy is validated in a data control sample that is nonoverlapping to the samples used in the search and in the background estimation described above. The validation sample uses the same selection as the search sample, but focuses on low- $p_{\mathrm{T}}^{\text {miss }}$ regions that are not utilized in the search. The requirement in high $\Delta m$ event categories of $m_{\mathrm{T}}^{\mathrm{b}} \geq 175 \mathrm{GeV}$ is also inverted when selecting events with at least two top quarks or $\mathrm{W}$ bosons to increase the statistical power of the validation exercise. Potential signal contamination in the validation regions is negligible. Figure 4 shows the predicted 

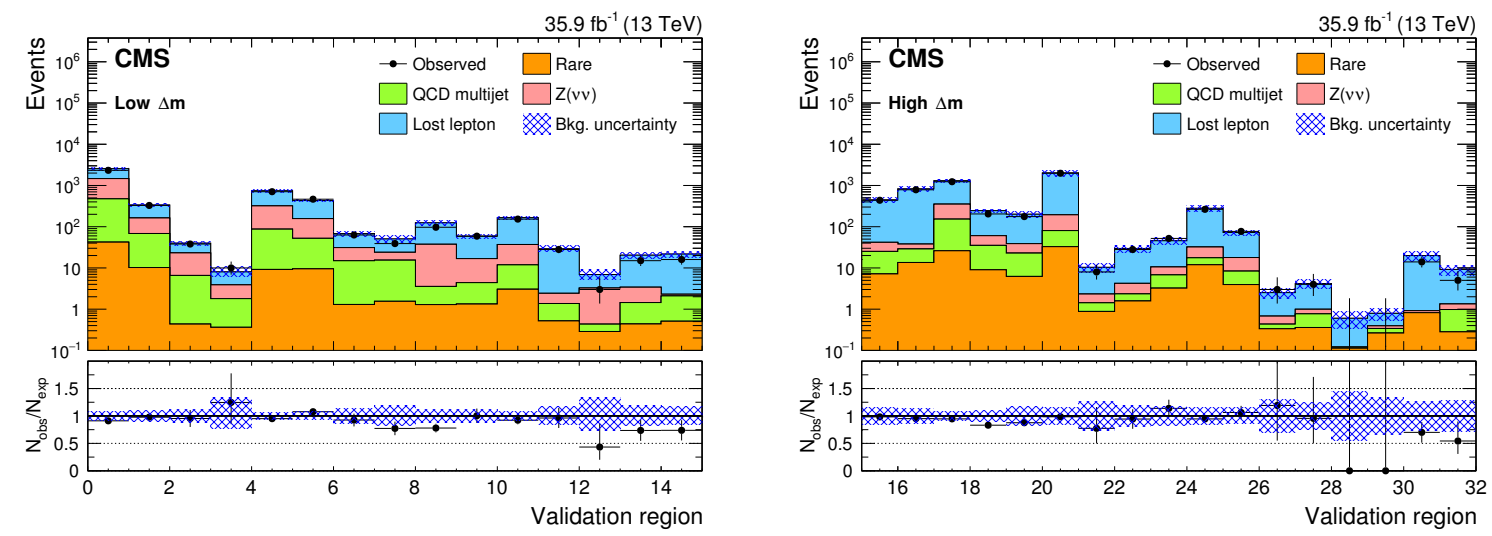

Figure 4. Observed data and SM background predictions in the low- $p_{\mathrm{T}}^{\mathrm{miss}}$ validation sample for the low $\Delta m$ (left) and high $\Delta m$ (right) selections. Ratios of the observed to SM predicted event counts derived from control regions are shown in the lower panel of each plot. The shaded blue band represents the statistical uncertainty combined with the systematic uncertainty resulting from the top quark and $\mathrm{W}$ boson tagging correction factors on the background prediction.

backgrounds and the observed data in the validation regions. The selections defining each bin are summarized in table 3. The SM prediction is consistent with the observed data, and no indication of a bias is found in the background estimation strategy.

\section{Systematic uncertainties}

As described in the preceding section, our strategy for estimating the background relies on translating event counts from data control regions to search regions by means of transfer factors obtained from simulation. These transfer factors, as well as the signal predictions, are therefore sensitive to a variety of systematic uncertainties related to the modeling of the experimental apparatus, particle kinematic properties, and theoretical models. We discuss the relevant sources of uncertainty below, and summarize their effects on the predictions in the SR in table 4.

- Choosing the size of control samples in data and simulation can lead to statistical limitations.

- Important systematic effects can arise from the dependence of the top quark and $\mathrm{W}$ boson tagging performance on the modeling of the t$\overline{\mathrm{t}}$ topology and showering of bottom quarks and partons from decays of $\mathrm{W}$ bosons to quarks. We assess a systematic uncertainty in the modeling of the $t \overline{\mathrm{t}}$ topology by comparing the tagging efficiencies in simulation between $t \bar{t}$ samples generated using POWHEG and MADGRAPH. The relative effect on the tagging efficiencies ranges from 1 to $4 \%$. Uncertainties related to the choice of scheme in parton showering are evaluated by comparing the tagging and mistagging efficiencies in simulation between the PYTHIA and HERWIG ++ shower models. Differences of 5 to $25 \%$ and 5 to $40 \%$ are seen for tagging and misidentification, respectively. We also evaluate the impact of heavy flavor jet multiplicity 


\begin{tabular}{|c|c|c|}
\hline Region & Selection & $p_{\mathrm{T}}^{\mathrm{miss}}[\mathrm{GeV}]$ \\
\hline 0 & $N_{\mathrm{b}}=0, N_{\mathrm{SV}}=0, p_{\mathrm{T}}^{\mathrm{ISR}} \geq 500 \mathrm{GeV}, 2 \leq N_{\mathrm{j}} \leq 5$ & $250-400$ \\
\hline 1 & $N_{\mathrm{b}}=0, N_{\mathrm{SV}}=0, p_{\mathrm{T}}^{\mathrm{ISR}} \geq 500 \mathrm{GeV}, N_{\mathrm{j}} \geq 6$ & $250-400$ \\
\hline 2 & $N_{\mathrm{b}}=0, N_{\mathrm{SV}} \geq 1, p_{\mathrm{T}}^{\mathrm{ISR}} \geq 500 \mathrm{GeV}, 2 \leq N_{\mathrm{j}} \leq 5$ & $250-400$ \\
\hline 3 & $N_{\mathrm{b}}=0, N_{\mathrm{SV}} \geq 1, p_{\mathrm{T}}^{\mathrm{ISR}} \geq 500 \mathrm{GeV}, N_{\mathrm{j}} \geq 6$ & $250-400$ \\
\hline 4 & $N_{\mathrm{b}}=1, N_{\mathrm{SV}}=0,300 \leq p_{\mathrm{T}}^{\mathrm{ISR}}<500 \mathrm{GeV}, p_{\mathrm{T}}^{\mathrm{b}}<40 \mathrm{GeV}$ & $250-300$ \\
\hline 5 & $N_{\mathrm{b}}=1, N_{\mathrm{SV}}=0,300 \leq p_{\mathrm{T}}^{\mathrm{ISR}}<500 \mathrm{GeV}, 40 \leq p_{\mathrm{T}}^{\mathrm{b}}<70 \mathrm{GeV}$ & $250-300$ \\
\hline 6 & $N_{\mathrm{b}}=1, N_{\mathrm{SV}}=0, p_{\mathrm{T}}^{\mathrm{ISR}} \geq 500 \mathrm{GeV}, p_{\mathrm{T}}^{\mathrm{b}}<40 \mathrm{GeV}$ & $250-400$ \\
\hline 7 & $N_{\mathrm{b}}=1, N_{\mathrm{SV}}=0, p_{\mathrm{T}}^{\mathrm{ISR}} \geq 500 \mathrm{GeV}, 40 \leq p_{\mathrm{T}}^{\mathrm{b}}<70 \mathrm{GeV}$ & $250-400$ \\
\hline 8 & $N_{\mathrm{b}}=1, N_{\mathrm{SV}} \geq 1, p_{\mathrm{T}}^{\mathrm{b}}<40 \mathrm{GeV}$ & $250-300$ \\
\hline 9 & $N_{\mathrm{b}} \geq 2,300 \leq p_{\mathrm{T}}^{\mathrm{ISR}}<500 \mathrm{GeV}, p_{\mathrm{T}}^{\mathrm{b}}<80 \mathrm{GeV}$ & $250-300$ \\
\hline 10 & $N_{\mathrm{b}} \geq 2,300 \leq p_{\mathrm{T}}^{\mathrm{ISR}}<500 \mathrm{GeV}, 80 \leq p_{\mathrm{T}}^{\mathrm{b}}<140 \mathrm{GeV}$ & $250-300$ \\
\hline 11 & $N_{\mathrm{b}} \geq 2,300 \leq p_{\mathrm{T}}^{\mathrm{ISR}}<500 \mathrm{GeV}, p_{\mathrm{T}}^{\mathrm{b}} \geq 140 \mathrm{GeV}, N_{\mathrm{j}} \geq 7$ & $250-300$ \\
\hline 12 & $N_{\mathrm{b}} \geq 2, p_{\mathrm{T}}^{\mathrm{ISR}} \geq 500 \mathrm{GeV}, p_{\mathrm{T}}^{\mathrm{b}}<80 \mathrm{GeV}$ & $250-400$ \\
\hline 13 & $N_{\mathrm{b}} \geq 2, p_{\mathrm{T}}^{\mathrm{ISR}} \geq 500 \mathrm{GeV}, 80 \leq p_{\mathrm{T}}^{\mathrm{b}}<140 \mathrm{GeV}$ & $250-400$ \\
\hline 14 & $N_{\mathrm{b}} \geq 2, p_{\mathrm{T}}^{\mathrm{ISR}} \geq 500 \mathrm{GeV}, p_{\mathrm{T}}^{\mathrm{b}} \geq 140 \mathrm{GeV}, N_{\mathrm{j}} \geq 7$ & $250-400$ \\
\hline 15 & $N_{\mathrm{b}}=1, m_{\mathrm{T}}^{\mathrm{b}}<175 \mathrm{GeV}, N_{\mathrm{j}} \geq 7, N_{\text {res }} \geq 1$ & $200-250$ \\
\hline 16 & $N_{\mathrm{b}} \geq 2, m_{\mathrm{T}}^{\mathrm{b}}<175 \mathrm{GeV}, N_{\mathrm{j}} \geq 7, N_{\mathrm{res}} \geq 1$ & $200-250$ \\
\hline 17 & $N_{\mathrm{b}}=1, N_{\mathrm{j}} \geq 7, N_{\mathrm{t}}=0, N_{\text {res }}=0, N_{\mathrm{W}}=0$ & $200-250$ \\
\hline 18 & $N_{\mathrm{b}} \geq 2, N_{\mathrm{j}} \geq 7, N_{\mathrm{t}}=0, N_{\text {res }}=0, N_{\mathrm{W}}=0$ & $200-250$ \\
\hline 19 & $N_{\mathrm{b}}=1, N_{\mathrm{t}} \geq 1, N_{\mathrm{res}}=0, N_{\mathrm{W}}=0$ & $200-450$ \\
\hline 20 & $N_{\mathrm{b}}=1, N_{\mathrm{t}}=0, N_{\mathrm{res}} \geq 1, N_{\mathrm{W}}=0$ & $200-250$ \\
\hline 21 & $N_{\mathrm{b}}=1, N_{\mathrm{t}} \geq 1, N_{\text {res }}=0, N_{\mathrm{W}} \geq 1$ & $200-450$ \\
\hline 22 & $N_{\mathrm{b}}=1, N_{\mathrm{t}}=0, N_{\mathrm{res}} \geq 1, N_{\mathrm{W}} \geq 1$ & $200-250$ \\
\hline 23 & $N_{\mathrm{b}} \geq 2, N_{\mathrm{t}}=1, N_{\mathrm{res}}=0, N_{\mathrm{W}}=0$ & $200-450$ \\
\hline 24 & $N_{\mathrm{b}} \geq 2, N_{\mathrm{t}}=0, N_{\mathrm{res}}=1, N_{\mathrm{W}}=0$ & $200-250$ \\
\hline 25 & $N_{\mathrm{b}} \geq 2, N_{\mathrm{t}}=0, N_{\mathrm{res}}=0, N_{\mathrm{W}}=1$ & $200-250$ \\
\hline 26 & $N_{\mathrm{b}} \geq 2, N_{\mathrm{t}}=1, N_{\mathrm{res}}=0, N_{\mathrm{W}}=1$ & $200-450$ \\
\hline 27 & $N_{\mathrm{b}} \geq 2, N_{\mathrm{t}}=0, N_{\mathrm{res}}=1, N_{\mathrm{W}}=1$ & $200-250$ \\
\hline 28 & $N_{\mathrm{b}} \geq 2, N_{\mathrm{t}}=1, N_{\mathrm{res}}=1, N_{\mathrm{W}}=0$ & $200-250$ \\
\hline 29 & $N_{\mathrm{b}} \geq 2, N_{\mathrm{t}} \geq 2, N_{\text {res }}=0, N_{\mathrm{W}}=0, m_{\mathrm{T}}^{\mathrm{b}}<175 \mathrm{GeV}$ or $p_{\mathrm{T}}^{\text {miss }}<250 \mathrm{GeV}$ & $\geq 200$ \\
\hline 30 & $N_{\mathrm{b}} \geq 2, N_{\mathrm{t}}=0, N_{\text {res }} \geq 2, N_{\mathrm{W}}=0, m_{\mathrm{T}}^{\mathrm{b}}<175 \mathrm{GeV}$ & $200-250$ \\
\hline 31 & $N_{\mathrm{b}} \geq 2, N_{\mathrm{t}}=0, N_{\text {res }}=0, N_{\mathrm{W}} \geq 2, m_{\mathrm{T}}^{\mathrm{b}}<175 \mathrm{GeV}$ & $200-250$ \\
\hline
\end{tabular}

Table 3. Summary of the validation region selections. The top part of the table (rows 0-14) corresponds to regions for the low $\Delta m$ selections, whereas the bottom part (rows 15-31) corresponds to regions for the high $\Delta m$ selections. 
on the data control sample used to define the misidentification correction factors by comparing the selection for $N_{\mathrm{b}}=0$ to the nominal selection of $N_{\mathrm{b}} \geq 1$. The observed difference of $20 \%$ is assigned as a systematic uncertainty.

The statistical precision in the tagging correction factors of the top quarks and $\mathrm{W}$ bosons ranges from 1 to $25 \%$. The definition of the generator-level matching scheme used when applying the tagging correction factors, as well as top $p_{\mathrm{T}}$ reweighting, are found to have very small impact on the top quark and $\mathrm{W}$ boson tagging performance. The sources of uncertainty discussed in this paragraph are collectively referred to as the "remaining sources" in table 4.

- In simulating rare background processes and signal, a $16 \%$ uncertainty is assessed to account for simulation-to-data differences in soft $\mathrm{b}$ tagging performance based on comparisons in control regions.

- Correction factors applied to simulation to account for differences in lepton selection efficiency between data and simulation have associated systematic uncertainties related to changes in the performance of the tracker over the data-taking period.

- Changes in $\mu_{\mathrm{R}}$ and $\mu_{\mathrm{F}}$ scales, PDF, and the strong coupling strength, $\alpha_{S}$ impact rare background predictions more significantly than background estimation from control samples in data that often benefit from partial or full cancellation of these uncertainties. The effect of unknown higher-order effects on any predicted event count is estimated by varying simultaneously $\mu_{\mathrm{R}}$ and $\mu_{\mathrm{F}}$ by a factor of two, as detailed in refs. [100, 101], and ranges from 1 to $10 \%$. The uncertainty related to the choice of PDF, including the uncertainty in $\alpha_{S}$, is obtained as the standard deviation in 100 variations of the NNPDF3.0 [54] PDF, and ranges from 1 to 28\%. The PDF systematics are evaluated only for background processes.

- A $2.5 \%$ uncertainty is assigned to the integrated luminosity measured by the CMS experiment for the 2016 data-taking period [102], and affects the simulation-based prediction of rare SM background and signal processes.

- A variety of other sources of systematic uncertainties include the corrections for $\mathrm{b}$ tagging performance, jet energy scale and resolution, which also affect the $p_{\mathrm{T}}^{\text {miss }}$ in the event, and reweighting of events for pileup. These sources are generally of much smaller importance compared to the other sources.

The magnitude of the systematic uncertainties is typically small, except for the most stringently defined SR affected by larger statistical uncertainties in the corresponding data control samples. The background estimation strategy, which translates event counts from data control samples with kinematic properties very similar to the corresponding SR, benefits from partial or full cancellation of many of the above sources of systematic uncertainty. 


\begin{tabular}{|c|c|c|c|c|c|}
\hline Source & Lost lepton & $\mathrm{Z}(\nu \nu)$ & QCD multijet & Rare & Signal \\
\hline $\mathrm{e} / \mu$ veto & $1-5$ & $2-3$ & $1-6$ & $1-8$ & $1-5$ \\
\hline$\tau_{\mathrm{h}}$ veto & $1-7$ & $2-3$ & $1-7$ & $1-7$ & $1-8$ \\
\hline b tagging: heavy flavor & $1-4$ & $1-5$ & $1-14$ & $1-6$ & $1-10$ \\
\hline b tagging: light flavor & $1-7$ & $1-15$ & $1-16$ & $1-8$ & $1-20$ \\
\hline Soft $b$ tagging & - & - & - & $1-16$ & $1-16$ \\
\hline Jet energy scale & $1-30$ & $1-25$ & $1-6$ & $1-31$ & $1-35$ \\
\hline$p_{\mathrm{T}}^{\text {miss }}$ resolution & $1-13$ & $1-18$ & $1-5$ & $1-30$ & $1-48$ \\
\hline $\mathrm{t} \overline{\mathrm{t}}$ normalization & $1-8$ & - & - & - & - \\
\hline $\mathrm{W}+$ jets normalization & $1-11$ & - & - & - & - \\
\hline Top quark $p_{\mathrm{T}}$ & $1-24$ & - & - & - & - \\
\hline Sample size (MC) & $1-100$ & $1-100$ & $2-100$ & $4-100$ & $2-100$ \\
\hline Sample size (data CR) & $2-100$ & $1-100$ & $3-100$ & - & - \\
\hline$R_{\mathrm{Z}}$ & - & $1-29$ & - & - & - \\
\hline $\mathrm{Z} / \gamma$ difference & - & $1-23$ & - & - & - \\
\hline Background subtraction & - & - & $13-71$ & - & - \\
\hline Jet response tail & - & - & $1-14$ & - & - \\
\hline$p_{\mathrm{T}}^{\text {miss }}$ integration & - & - & $1-51$ & - & - \\
\hline Smearing closure & - & - & 50 & - & - \\
\hline Pileup reweighting & $1-12$ & $1-12$ & $1-12$ & $1-16$ & $1-15$ \\
\hline Integrated luminosity & - & - & - & 2.5 & 2.5 \\
\hline Cross section & - & - & - & $24-50$ & - \\
\hline PDF and $\alpha_{S}$ dependence & $1-10$ & - & - & $1-10$ & - \\
\hline$\mu_{\mathrm{R}} / \mu_{\mathrm{F}}$ dependence & $1-7$ & - & - & $1-28$ & $1-9$ \\
\hline \multicolumn{6}{|l|}{ Merged tagging } \\
\hline Generator & $<3$ & - & - & $<4$ & $<4$ \\
\hline Parton showering & $1-22$ & $1-23$ & $1-42$ & $1-25$ & $1-34$ \\
\hline Sample size (data) & $1-5$ & $1-7$ & $1-11$ & $1-3$ & $1-3$ \\
\hline Mistag $N_{\mathrm{b}}$ & $1-22$ & $1-18$ & $1-18$ & $1-6$ & $1-5$ \\
\hline Remaining sources & $1-24$ & - & - & $1-27$ & $1-32$ \\
\hline \multicolumn{6}{|l|}{ Resolved tagging } \\
\hline Generator & $<1$ & - & - & $<1$ & $<3$ \\
\hline Parton showering & $1-12$ & - & - & $1-16$ & $1-31$ \\
\hline Remaining sources & $1-18$ & $1-17$ & $1-17$ & $1-16$ & $1-20$ \\
\hline
\end{tabular}

Table 4. Range of systematic uncertainties [\%] in the prediction across the different search regions. "Rare" column includes diboson and t⿱一兀Z processes. "Signal" column shows the range of systematic uncertainties representative of the full set of models shown in figure 1. 


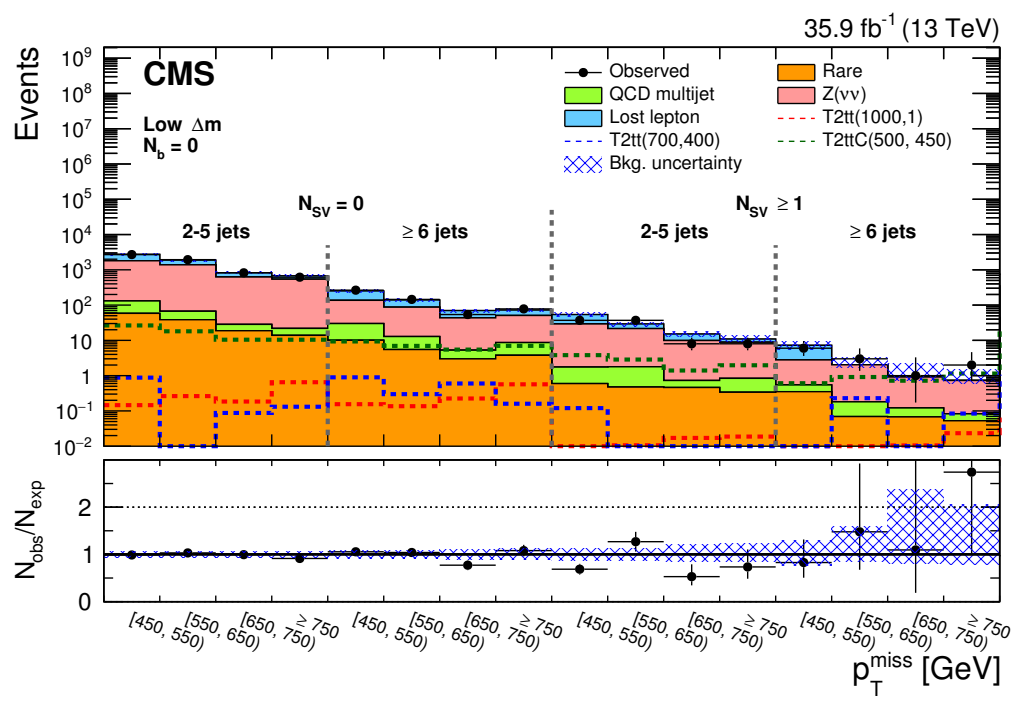

Figure 5. Observed data events and SM background predictions for the low $\Delta m$ search regions with $N_{\mathrm{b}}=0$. Ratios of the observed to SM predicted event counts are shown in the lower panel of each plot. The shaded blue band represents the combined statistical and systematic uncertainties on the SM predictions. Units are $\mathrm{GeV}$.

\section{Results and interpretation}

The event counts observed in data and those predicted for SM backgrounds are summarized graphically in figures 5-8, and numerically in tables 5 and 6 . The observed event counts are in general agreement with the predictions. The two search regions with most significant discrepancies are the low $\Delta m$ SR defined by the selection $N_{\mathrm{b}} \geq 2, m_{\mathrm{T}}^{\mathrm{b}}<175 \mathrm{GeV}, p_{\mathrm{T}}^{\mathrm{ISR}} \geq$ $500 \mathrm{GeV}, p_{\mathrm{T}}^{\mathrm{b}}<80 \mathrm{GeV}, p_{\mathrm{T}}^{\text {miss }} \geq 650 \mathrm{GeV}$, and the high $\Delta m$ SR defined by $N_{\mathrm{b}}=1, m_{\mathrm{T}}^{\mathrm{b}} \geq$ $175 \mathrm{GeV}, N_{\mathrm{t}} \geq 1, N_{\text {res }}=0, N_{\mathrm{W}} \geq 1, p_{\mathrm{T}}^{\text {miss }} \geq 550 \mathrm{GeV}$. For these two SR, observed excesses over the predicted event counts correspond to local significances of 2.3 and 1.9 standard deviations, respectively. These can be attributed to statistical fluctuations of the SM backgrounds alone, given the number of search regions employed in this analysis.

The statistical interpretations of the results in terms of exclusion limits for signal models being considered in this analysis are based on a binned likelihood fit to the data, which takes into account the predicted background and signal yields in the SR. The extraction of exclusion limits is based on a modified frequentist approach using the $\mathrm{CL}_{\mathrm{s}}$ criterion $[103,104]$ under the asymptotic approximation for the test statistic [105, 106]. All of the SR, and their corresponding CR, are fitted simultaneously to determine the signal cross section excluded at a $95 \%$ confidence level (CL) for each signal point. Models for signal in which the $95 \%$ CL upper limit on the production cross section falls below the theoretical value (based on NLO+NLL calculations) are considered excluded by this analysis.

The inclusion of the single-lepton CR in the likelihood fit ensures that any signal contamination in the $\mathrm{CR}$ is taken into account, through estimates of the corresponding signal, by the fit. The systematic uncertainties assigned to the signal and background are treated as nuisance parameters in the fit. The term "nuisance parameter" refers to a 


\begin{tabular}{|c|c|c|c|c|c|c|}
\hline$p_{\mathrm{T}}^{\text {miss }}[\mathrm{GeV}]$ & Lost lepton & $\mathrm{Z}(\nu \nu)$ & Rare & QCD multijet & Total SM & $N_{\text {data }}$ \\
\hline \multicolumn{7}{|c|}{$N_{\mathrm{b}}=0, N_{\mathrm{SV}}=0, p_{\mathrm{T}}^{\mathrm{ISR}} \geq 500 \mathrm{GeV}, 2 \leq N_{\mathrm{j}} \leq 5$} \\
\hline $450-550$ & $935 \pm 73$ & $1670 \pm 120$ & $58 \pm 29$ & $73 \pm 37$ & $2740 \pm 180$ & 2704 \\
\hline $550-650$ & $498 \pm 39$ & $1318 \pm 84$ & $38 \pm 19$ & $28 \pm 14$ & $1880 \pm 110$ & 1942 \\
\hline $650-750$ & $202 \pm 19$ & $597 \pm 43$ & $19 \pm 10$ & $9.6 \pm 4.9$ & $828 \pm 55$ & 823 \\
\hline$\geq 750$ & $135 \pm 14$ & $520 \pm 38$ & $14 \pm 7$ & $7.9 \pm 4.2$ & $676 \pm 46$ & 618 \\
\hline \multicolumn{7}{|c|}{$N_{\mathrm{b}}=0, N_{\mathrm{SV}}=0, p_{\mathrm{T}}^{\mathrm{ISR}} \geq 500 \mathrm{GeV}, N_{\mathrm{j}} \geq 6$} \\
\hline $450-550$ & $115 \pm 12$ & $106 \pm 10$ & $10 \pm 5$ & $20 \pm 10$ & $251 \pm 22$ & 265 \\
\hline $550-650$ & $52 \pm 6$ & $74 \pm 7$ & $5.5 \pm 2.8$ & $7.3 \pm 3.8$ & $139 \pm 12$ & 145 \\
\hline $650-750$ & $27 \pm 4$ & $38 \pm 5$ & $3.0 \pm 1.6$ & $2.3 \pm 1.3$ & $70 \pm 7$ & 54 \\
\hline$\geq 750$ & $21 \pm 4$ & $42 \pm 5$ & $3.8 \pm 2.0$ & $4.9_{-5.2}^{+6.3}$ & $72_{-8}^{+10}$ & 78 \\
\hline \multicolumn{7}{|c|}{$N_{\mathrm{b}}=0, N_{\mathrm{SV}} \geq 1, p_{\mathrm{T}}^{\mathrm{ISR}} \geq 500 \mathrm{GeV}, 2 \leq N_{\mathrm{j}} \leq 5$} \\
\hline $450-550$ & $25 \pm 5$ & $27 \pm 3$ & $0.60 \pm 0.47$ & $1.2 \pm 0.7$ & $54 \pm 6$ & 37 \\
\hline $550-650$ & $7.6 \pm 2.5$ & $20 \pm 2$ & $0.47 \pm 0.37$ & $1.3_{-0.9}^{+1.2}$ & $29 \pm 4$ & 37 \\
\hline $650-750$ & $5.2_{-1.9}^{+2.7}$ & $9.2 \pm 1.1$ & $0.46 \pm 0.40$ & $0.27_{-0.24}^{+0.29}$ & $15_{-2}^{+3}$ & 8 \\
\hline$\geq 750$ & $2.0_{-1.1}^{+2.0}$ & $8.0 \pm 1.0$ & $0.34 \pm 0.26$ & $0.50_{-0.34}^{+0.40}$ & $11 \pm 2$ & 8 \\
\hline \multicolumn{7}{|c|}{$N_{\mathrm{b}}=0, N_{\mathrm{SV}} \geq 1, p_{\mathrm{T}}^{\mathrm{ISR}} \geq 500 \mathrm{GeV}, N_{\mathrm{j}} \geq 6$} \\
\hline $450-550$ & $4.5_{-1.6}^{+2.1}$ & $2.2 \pm 0.4$ & $0.35 \pm 0.29$ & $0.19_{-0.13}^{+0.17}$ & $7.2_{-1.7}^{+2.2}$ & 6 \\
\hline $550-650$ & $<1.08$ & $1.8 \pm 0.3$ & $0.07 \pm 0.05$ & $0.11_{-0.08}^{+0.10}$ & $2.0_{-0.3}^{+1.2}$ & 3 \\
\hline $650-750$ & $<1.22$ & $0.79 \pm 0.17$ & $0.07 \pm 0.05$ & $0.05_{-0.04}^{+0.05}$ & $0.9_{-0.2}^{+1.3}$ & 1 \\
\hline$\geq 750$ & $<0.74$ & $0.65 \pm 0.14$ & $0.05 \pm 0.05$ & $0.03_{-0.02}^{+0.03}$ & $0.73_{-0.15}^{+0.77}$ & 2 \\
\hline \multicolumn{7}{|c|}{$N_{\mathrm{b}}=1, N_{\mathrm{SV}}=0, m_{\mathrm{T}}^{\mathrm{b}}<175 \mathrm{GeV}, 300 \leq p_{\mathrm{T}}^{\mathrm{ISR}}<500 \mathrm{GeV}, p_{\mathrm{T}}^{\mathrm{b}}<40 \mathrm{GeV}$} \\
\hline $300-400$ & $410 \pm 38$ & $318 \pm 29$ & $14 \pm 7$ & $32 \pm 17$ & $774 \pm 57$ & 753 \\
\hline $400-500$ & $64 \pm 11$ & $77 \pm 10$ & $3.8 \pm 1.9$ & $6.3 \pm 3.9$ & $151 \pm 16$ & 147 \\
\hline $500-600$ & $4.7_{-2.4}^{+3.9}$ & $7.6 \pm 2.2$ & $0.5 \pm 0.3$ & $0.83 \pm 0.59$ & $14_{-3}^{+5}$ & 13 \\
\hline$\geq 600$ & $2.4_{-1.3}^{+2.1}$ & $0.34_{-0.28}^{+0.79}$ & $0.11 \pm 0.07$ & $0.14 \pm 0.11$ & $2.9_{-1.4}^{+2.5}$ & 5 \\
\hline \multicolumn{7}{|c|}{$N_{\mathrm{b}}=1, N_{\mathrm{SV}}=0, m_{\mathrm{T}}^{\mathrm{b}}<175 \mathrm{GeV}, 300 \leq p_{\mathrm{T}}^{\mathrm{ISR}}<500 \mathrm{GeV}, 40 \leq p_{\mathrm{T}}^{\mathrm{b}}<70 \mathrm{GeV}$} \\
\hline $300-400$ & $285 \pm 33$ & $140 \pm 15$ & $8.3 \pm 3.8$ & $8.6 \pm 4.7$ & $442 \pm 39$ & 375 \\
\hline $400-500$ & $50 \pm 10$ & $23 \pm 4$ & $1.7 \pm 0.9$ & $2.1 \pm 1.5$ & $76 \pm 11$ & 76 \\
\hline $500-600$ & $6.4_{-2.9}^{+4.2}$ & $2.3_{-1.0}^{+1.5}$ & $0.22 \pm 0.13$ & $0.08 \pm 0.06$ & $9.0_{-3.1}^{+4.8}$ & 5 \\
\hline$\geq 600$ & $<0.83$ & $1.6_{-1.1}^{+1.9}$ & $0.02 \pm 0.03$ & $0.02 \pm 0.02$ & $1.7_{-1.1}^{+2.4}$ & 0 \\
\hline \multicolumn{7}{|c|}{$N_{\mathrm{b}}=1, N_{\mathrm{SV}}=0, m_{\mathrm{T}}^{\mathrm{b}}<175 \mathrm{GeV}, p_{\mathrm{T}}^{\mathrm{ISR}} \geq 500 \mathrm{GeV}, p_{\mathrm{T}}^{\mathrm{b}}<40 \mathrm{GeV}$} \\
\hline $450-550$ & $31 \pm 6$ & $19 \pm 4$ & $1.9 \pm 1.1$ & $2.0 \pm 1.2$ & $54 \pm 8$ & 41 \\
\hline $550-650$ & $9.3 \pm 3.0$ & $7.8 \pm 2.0$ & $0.62 \pm 0.42$ & $0.57_{-0.40}^{+0.48}$ & $18 \pm 4$ & 24 \\
\hline $650-750$ & $1.7_{-1.1}^{+2.3}$ & $7.5 \pm 2.2$ & $0.01 \pm 0.17$ & $0.06_{-0.05}^{+0.06}$ & $9.3_{-2.5}^{+3.5}$ & 7 \\
\hline$\geq 750$ & $<1.48$ & $4.0_{-1.5}^{+2.1}$ & $0.16 \pm 0.10$ & $0.11_{-0.08}^{+0.00}$ & $4.2_{-1.5}^{+3.2}$ & 4 \\
\hline
\end{tabular}




\begin{tabular}{|c|c|c|c|c|c|c|}
\hline$p_{\mathrm{T}}^{\text {miss }}[\mathrm{GeV}]$ & Lost lepton & $\mathrm{Z}(\nu \nu)$ & Rare & QCD multijet & Total SM & $N_{\text {data }}$ \\
\hline \multicolumn{7}{|c|}{$N_{\mathrm{b}}=1, N_{\mathrm{SV}}=0, m_{\mathrm{T}}^{\mathrm{b}}<175 \mathrm{GeV}, p_{\mathrm{T}}^{\mathrm{ISR}} \geq 500 \mathrm{GeV}, 40 \leq p_{\mathrm{T}}^{\mathrm{b}}<70 \mathrm{GeV}$} \\
\hline $450-550$ & $22 \pm 5$ & $6.6 \pm 1.7$ & $1.4 \pm 0.8$ & $1.3 \pm 0.8$ & $31 \pm 5$ & 18 \\
\hline $550-650$ & $11_{-4}^{+6}$ & $5.5 \pm 1.8$ & $0.31 \pm 0.18$ & $0.17_{-0.12}^{+0.16}$ & $17_{-5}^{+6}$ & 23 \\
\hline $650-750$ & $3.0_{-1.6}^{+2.6}$ & $2.5_{-1.3}^{+1.9}$ & $0.08 \pm 0.09$ & $0.06_{-0.06}^{+0.10}$ & $5.6_{-2.2}^{+3.7}$ & 4 \\
\hline$\geq 750$ & $1.7_{-1.1}^{+2.3}$ & $3.1_{-1.5}^{+2.1}$ & $0.14 \pm 0.09$ & $0.07_{-0.06}^{+0.11}$ & $4.9_{-1.9}^{+3.6}$ & 3 \\
\hline \multicolumn{7}{|c|}{$N_{\mathrm{b}}=1, N_{\mathrm{SV}} \geq 1, m_{\mathrm{T}}^{\mathrm{b}}<175 \mathrm{GeV}, p_{\mathrm{T}}^{\mathrm{b}}<40 \mathrm{GeV}$} \\
\hline $300-400$ & $38 \pm 8$ & $16 \pm 5$ & $1.1 \pm 0.6$ & $1.0_{-0.8}^{+1.0}$ & $56_{-9}^{+10}$ & 44 \\
\hline $400-500$ & $4.9_{-2.5}^{+3.8}$ & $2.9 \pm 1.0$ & $0.16 \pm 0.13$ & $0.58_{-0.54}^{+0.97}$ & $8.6_{-2.8}^{+4.4}$ & 6 \\
\hline$\geq 500$ & $1.4_{-1.0}^{+1.9}$ & $0.86 \pm 0.31$ & $0.03 \pm 0.03$ & $0.04_{-0.04}^{+0.08}$ & $2.3_{-1.0}^{+2.0}$ & 4 \\
\hline \multicolumn{7}{|c|}{$N_{\mathrm{b}} \geq 2, m_{\mathrm{T}}^{\mathrm{b}}<175 \mathrm{GeV}, 300 \leq p_{\mathrm{T}}^{\mathrm{ISR}}<500 \mathrm{GeV}, p_{\mathrm{T}}^{\mathrm{b}}<80 \mathrm{GeV}$} \\
\hline $300-400$ & $47 \pm 8$ & $16 \pm 5$ & $2.2 \pm 1.0$ & $2.0_{-1.5}^{+1.8}$ & $68_{-9}^{+10}$ & 57 \\
\hline $400-500$ & $6.7_{-2.6}^{+3.4}$ & $5.5 \pm 2.4$ & $0.39 \pm 0.23$ & $0.19_{-0.16}^{+0.18}$ & $13 \pm 4$ & 7 \\
\hline$\geq 500$ & $3.6_{-2.7}^{+4.3}$ & $0.7_{-0.6}^{+1.1}$ & $0.08 \pm 0.05$ & $<0.01$ & $4.4_{-2.7}^{+4.7}$ & 1 \\
\hline \multicolumn{7}{|c|}{$N_{\mathrm{b}} \geq 2, m_{\mathrm{T}}^{\mathrm{b}}<175 \mathrm{GeV}, 300 \leq p_{\mathrm{T}}^{\mathrm{ISR}}<500 \mathrm{GeV}, 80 \leq p_{\mathrm{T}}^{\mathrm{b}}<140 \mathrm{GeV}$} \\
\hline $300-400$ & $121 \pm 13$ & $20 \pm 5$ & $4.2 \pm 1.7$ & $4.2 \pm 2.5$ & $149 \pm 15$ & 149 \\
\hline $400-500$ & $21 \pm 5$ & $5.5 \pm 2.0$ & $1.2 \pm 0.6$ & $0.9_{-0.9}^{+1.6}$ & $28_{-5}^{+6}$ & 19 \\
\hline$\geq 500$ & $1.7_{-1.0}^{+1.8}$ & $1.6_{-1.0}^{+1.6}$ & $0.27 \pm 0.16$ & $0.01 \pm 0.01$ & $3.6_{-1.5}^{+2.8}$ & 4 \\
\hline \multicolumn{7}{|c|}{$N_{\mathrm{b}} \geq 2, m_{\mathrm{T}}^{\mathrm{b}}<175 \mathrm{GeV}, 300 \leq p_{\mathrm{T}}^{\mathrm{ISR}}<500 \mathrm{GeV}, p_{\mathrm{T}}^{\mathrm{b}}>140 \mathrm{GeV}, N_{\mathrm{j}} \geq 7$} \\
\hline $300-400$ & $52 \pm 8$ & $3.5_{-1.4}^{+1.9}$ & $1.4 \pm 0.6$ & $2.9 \pm 1.8$ & $60 \pm 8$ & 54 \\
\hline $400-500$ & $13 \pm 3$ & $0.7_{-0.5}^{+1.0}$ & $0.41 \pm 0.16$ & $0.18_{-0.18}^{+0.45}$ & $15_{-3}^{+4}$ & 12 \\
\hline$\geq 500$ & $1.8_{-1.1}^{+1.9}$ & $0.5_{-0.4}^{+1.2}$ & $0.04 \pm 0.15$ & $0.07_{-0.07}^{+0.19}$ & $2.4_{-1.2}^{+2.7}$ & 6 \\
\hline \multicolumn{7}{|c|}{$N_{\mathrm{b}} \geq 2, m_{\mathrm{T}}^{\mathrm{b}}<175 \mathrm{GeV}, p_{\mathrm{T}}^{\mathrm{ISR}} \geq 500 \mathrm{GeV}, p_{\mathrm{T}}^{\mathrm{b}}<80 \mathrm{GeV}$} \\
\hline $450-550$ & $2.5_{-1.4}^{+2.2}$ & $0.52_{-0.31}^{+0.46}$ & $0.15 \pm 0.08$ & $0.1_{-0.09}^{+0.13}$ & $3.3_{-1.5}^{+2.4}$ & 6 \\
\hline $550-650$ & $<1.59$ & $1.4_{-0.9}^{+1.5}$ & $0.02 \pm 0.06$ & $0.05_{-0.04}^{+0.07}$ & $1.4_{-0.9}^{+2.7}$ & 2 \\
\hline$\geq 650$ & $<0.75$ & $<0.33$ & $0.15 \pm 0.14$ & $0.06_{-0.06}^{+0.09}$ & $0.2_{-0.2}^{+1.0}$ & 5 \\
\hline \multicolumn{7}{|c|}{$N_{\mathrm{b}} \geq 2, m_{\mathrm{T}}^{\mathrm{b}}<175 \mathrm{GeV}, p_{\mathrm{T}}^{\mathrm{ISR}} \geq 500 \mathrm{GeV}, 80 \leq p_{\mathrm{T}}^{\mathrm{b}}<140 \mathrm{GeV}$} \\
\hline $450-550$ & $6.4_{-2.2}^{+3.0}$ & $1.9_{-0.9}^{+1.3}$ & $0.33 \pm 0.22$ & $0.58_{-0.47}^{+0.57}$ & $9.2_{-2.5}^{+3.7}$ & 7 \\
\hline $550-650$ & $3.0_{-1.6}^{+2.6}$ & $0.63_{-0.44}^{+0.89}$ & $0.24 \pm 0.16$ & $0.07_{-0.05}^{+0.06}$ & $3.9_{-1.7}^{+3.0}$ & 1 \\
\hline$\geq 650$ & $0.7_{-0.6}^{+1.6}$ & $0.78_{-0.50}^{+0.87}$ & $0.30 \pm 0.23$ & $0.03_{-0.02}^{+0.03}$ & $1.8_{-0.9}^{+2.1}$ & 1 \\
\hline \multicolumn{7}{|c|}{$N_{\mathrm{b}} \geq 2, m_{\mathrm{T}}^{\mathrm{b}}<175 \mathrm{GeV}, p_{\mathrm{T}}^{\mathrm{ISR}} \geq 500 \mathrm{GeV}, p_{\mathrm{T}}^{\mathrm{b}}>140 \mathrm{GeV}, N_{\mathrm{j}} \geq 7$} \\
\hline $450-550$ & $12 \pm 3$ & $0.12_{-0.12}^{+0.34}$ & $0.34 \pm 0.19$ & $1.1_{-0.8}^{+0.9}$ & $13 \pm 3$ & 22 \\
\hline $550-650$ & $5.3_{-2.1}^{+2.8}$ & $0.29_{-0.25}^{+0.71}$ & $0.07 \pm 0.10$ & $0.36_{-0.25}^{+0.31}$ & $6.0_{-2.1}^{+3.2}$ & 5 \\
\hline$\geq 650$ & $4.4_{-2.4}^{+3.8}$ & $<0.85$ & $0.42 \pm 0.41$ & $0.14_{-0.1}^{+0.13}$ & $4.9_{-2.4}^{+4.3}$ & 1 \\
\hline
\end{tabular}

Table 5. Predicted background yields and the observation in different search regions for the low $\Delta m$ analysis. The total uncertainty is given for each background prediction. 


\begin{tabular}{|c|c|c|c|c|c|c|}
\hline$p_{\mathrm{T}}^{\mathrm{miss}}[\mathrm{GeV}]$ & Lost lepton & $\mathrm{Z}(\nu \nu)$ & Rare & QCD multijet & Total SM & $N_{\text {data }}$ \\
\hline \multicolumn{7}{|c|}{$N_{\mathrm{b}}=1, m_{\mathrm{T}}^{\mathrm{b}}<175 \mathrm{GeV}, N_{\mathrm{j}} \geq 7, N_{\text {res }} \geq 1$} \\
\hline $250-300$ & $136 \pm 23$ & $8.9 \pm 2.7$ & $3.4 \pm 0.9$ & $2.9 \pm 1.8$ & $151 \pm 26$ & 131 \\
\hline $300-400$ & $64 \pm 12$ & $4.8 \pm 1.4$ & $2.4 \pm 0.7$ & $1.7 \pm 1.1$ & $73 \pm 13$ & 73 \\
\hline $400-500$ & $8.5 \pm 2.1$ & $1.3 \pm 0.4$ & $0.57 \pm 0.22$ & $0.25 \pm 0.17$ & $11 \pm 2$ & 16 \\
\hline$\geq 500$ & $2.9 \pm 1.1$ & $0.54 \pm 0.23$ & $0.14 \pm 0.08$ & $0.05 \pm 0.03$ & $3.6 \pm 1.1$ & 0 \\
\hline \multicolumn{7}{|c|}{$N_{\mathrm{b}} \geq 2, m_{\mathrm{T}}^{\mathrm{b}}<175 \mathrm{GeV}, N_{\mathrm{j}} \geq 7, N_{\mathrm{res}} \geq 1$} \\
\hline $250-300$ & $274 \pm 42$ & $4.1 \pm 1.3$ & $6.8 \pm 2.0$ & $2.8 \pm 1.8$ & $288 \pm 44$ & 289 \\
\hline $300-400$ & $146 \pm 23$ & $2.7 \pm 0.9$ & $4.8 \pm 1.3$ & $1.3 \pm 0.9$ & $155 \pm 24$ & 131 \\
\hline $400-500$ & $21 \pm 4$ & $1.2 \pm 0.5$ & $1.3 \pm 0.4$ & $0.12 \pm 0.09$ & $23 \pm 4$ & 19 \\
\hline$\geq 500$ & $6.7 \pm 1.9$ & $0.49 \pm 0.22$ & $0.67 \pm 0.28$ & $0.03 \pm 0.02$ & $7.9 \pm 2.0$ & 9 \\
\hline \multicolumn{7}{|c|}{$N_{\mathrm{b}}=1, m_{\mathrm{T}}^{\mathrm{b}} \geq 175 \mathrm{GeV}, N_{\mathrm{j}} \geq 7, N_{\mathrm{t}}=0, N_{\mathrm{res}}=0, N_{\mathrm{W}}=0$} \\
\hline $250-350$ & $568 \pm 63$ & $200 \pm 18$ & $27 \pm 8$ & $104 \pm 69$ & $900 \pm 100$ & 899 \\
\hline $350-450$ & $141 \pm 17$ & $87 \pm 8$ & $10 \pm 3$ & $12 \pm 7$ & $251 \pm 23$ & 235 \\
\hline $450-550$ & $27 \pm 4$ & $40 \pm 6$ & $3.9 \pm 1.3$ & $3.6 \pm 2.1$ & $74 \pm 8$ & 62 \\
\hline$\geq 550$ & $20 \pm 4$ & $33 \pm 8$ & $3.8 \pm 1.6$ & $2.0 \pm 1.1$ & $59 \pm 10$ & 41 \\
\hline \multicolumn{7}{|c|}{$N_{\mathrm{b}} \geq 2, m_{\mathrm{T}}^{\mathrm{b}} \geq 175 \mathrm{GeV}, N_{\mathrm{j}} \geq 7, N_{\mathrm{t}}=0, N_{\mathrm{res}}=0, N_{\mathrm{W}}=0$} \\
\hline $250-350$ & $120 \pm 15$ & $45 \pm 6$ & $9.6 \pm 2.5$ & $14 \pm 8$ & $188 \pm 20$ & 174 \\
\hline $350-450$ & $28 \pm 5$ & $15 \pm 3$ & $4.2 \pm 1.2$ & $3.7 \pm 2.1$ & $51 \pm 7$ & 65 \\
\hline $450-550$ & $8.0 \pm 2.1$ & $7.2 \pm 1.6$ & $1.7 \pm 0.5$ & $1.0_{-0.7}^{+0.8}$ & $18 \pm 3$ & 22 \\
\hline$\geq 550$ & $4.2 \pm 1.3$ & $5.4 \pm 1.8$ & $1.1 \pm 0.4$ & $0.45_{-0.37}^{+0.47}$ & $11 \pm 2$ & 13 \\
\hline \multicolumn{7}{|c|}{$N_{\mathrm{b}}=1, m_{\mathrm{T}}^{\mathrm{b}} \geq 175 \mathrm{GeV}, N_{\mathrm{t}} \geq 1, N_{\mathrm{res}}=0, N_{\mathrm{W}}=0$} \\
\hline $550-650$ & $3.3 \pm 1.2$ & $2.3 \pm 0.7$ & $0.81 \pm 0.26$ & $0.08 \pm 0.06$ & $6.4 \pm 1.5$ & 6 \\
\hline$\geq 650$ & $2.6 \pm 1.0$ & $2.5 \pm 0.6$ & $0.62 \pm 0.20$ & $0.11 \pm 0.08$ & $5.9 \pm 1.3$ & 4 \\
\hline \multicolumn{7}{|c|}{$N_{\mathrm{b}}=1, m_{\mathrm{T}}^{\mathrm{b}} \geq 175 \mathrm{GeV}, N_{\mathrm{t}}=0, N_{\mathrm{res}} \geq 1, N_{\mathrm{W}}=0$} \\
\hline $250-350$ & $930 \pm 170$ & $110 \pm 28$ & $32 \pm 9$ & $17 \pm 10$ & $1090 \pm 180$ & 1120 \\
\hline $350-450$ & $128 \pm 28$ & $39 \pm 9$ & $13 \pm 4$ & $2.9 \pm 1.8$ & $183 \pm 33$ & 165 \\
\hline $450-550$ & $18 \pm 4$ & $14 \pm 3$ & $3.0 \pm 0.9$ & $1.5 \pm 1.1$ & $36 \pm 6$ & 41 \\
\hline $550-650$ & $3.3 \pm 1.1$ & $4.8 \pm 1.5$ & $1.4 \pm 0.5$ & $0.80 \pm 0.66$ & $10 \pm 2$ & 9 \\
\hline$\geq 650$ & $1.9 \pm 0.6$ & $3.2 \pm 0.8$ & $0.62 \pm 0.20$ & $0.13 \pm 0.11$ & $5.8 \pm 1.3$ & 8 \\
\hline \multicolumn{7}{|c|}{$N_{\mathrm{b}}=1, m_{\mathrm{T}}^{\mathrm{b}} \geq 175 \mathrm{GeV}, N_{\mathrm{t}} \geq 1, N_{\mathrm{res}}=0, N_{\mathrm{W}} \geq 1$} \\
\hline$\geq 550$ & $0.08 \pm 0.07$ & $0.11 \pm 0.08$ & $0.17 \pm 0.07$ & $0.01 \pm 0.01$ & $0.37 \pm 0.16$ & 3 \\
\hline \multicolumn{7}{|c|}{$N_{\mathrm{b}}=1, m_{\mathrm{T}}^{\mathrm{b}} \geq 175 \mathrm{GeV}, N_{\mathrm{t}}=0, N_{\mathrm{res}} \geq 1, N_{\mathrm{W}} \geq 1$} \\
\hline $250-350$ & $17 \pm 4$ & $1.7 \pm 0.6$ & $1.8 \pm 0.6$ & $0.46 \pm 0.39$ & $21 \pm 5$ & 19 \\
\hline $350-450$ & $4.1 \pm 1.4$ & $1.1 \pm 0.5$ & $0.79 \pm 0.26$ & $0.03 \pm 0.03$ & $6.0 \pm 1.7$ & 5 \\
\hline $450-550$ & $0.92 \pm 0.46$ & $0.34 \pm 0.14$ & $0.31 \pm 0.17$ & $0.16 \pm 0.18$ & $1.7 \pm 0.6$ & 3 \\
\hline$\geq 550$ & $0.45 \pm 0.27$ & $0.22 \pm 0.11$ & $0.42 \pm 0.31$ & $0.05 \pm 0.05$ & $1.1 \pm 0.5$ & 0 \\
\hline
\end{tabular}




\begin{tabular}{|c|c|c|c|c|c|c|}
\hline$p_{\mathrm{T}}^{\text {miss }}[\mathrm{GeV}]$ & Lost lepton & $\mathrm{Z}(\nu \nu)$ & Rare & QCD multijet & Total SM & $N_{\text {data }}$ \\
\hline \multicolumn{7}{|c|}{$N_{\mathrm{b}} \geq 2, m_{\mathrm{T}}^{\mathrm{b}} \geq 175 \mathrm{GeV}, N_{\mathrm{t}}=1, N_{\text {res }}=0, N_{\mathrm{W}}=0$} \\
\hline $550-650$ & $1.0 \pm 0.5$ & $0.48 \pm 0.19$ & $0.7 \pm 0.2$ & $0.03 \pm 0.03$ & $2.2 \pm 0.6$ & 2 \\
\hline$\geq 650$ & $0.38_{-0.22}^{+0.27}$ & $0.71 \pm 0.23$ & $0.56 \pm 0.17$ & $0.03_{-0.02}^{+0.03}$ & $1.7 \pm 0.4$ & 4 \\
\hline \multicolumn{7}{|c|}{$N_{\mathrm{b}} \geq 2, m_{\mathrm{T}}^{\mathrm{b}} \geq 175 \mathrm{GeV}, N_{\mathrm{t}}=0, N_{\text {res }}=1, N_{\mathrm{W}}=0$} \\
\hline $250-350$ & $148 \pm 26$ & $24 \pm 6$ & $16 \pm 4$ & $6.2 \pm 4.3$ & $194 \pm 32$ & 175 \\
\hline $350-450$ & $23 \pm 5$ & $7.2 \pm 1.8$ & $7.3 \pm 2.0$ & $1.2 \pm 0.8$ & $38 \pm 7$ & 38 \\
\hline $450-550$ & $3.6 \pm 1.1$ & $3.6 \pm 1.0$ & $2.3 \pm 0.6$ & $0.46 \pm 0.40$ & $9.9 \pm 2.0$ & 7 \\
\hline $550-650$ & $1.6 \pm 0.6$ & $1.4 \pm 0.5$ & $0.76 \pm 0.25$ & $0.12 \pm 0.13$ & $3.9 \pm 1.0$ & 1 \\
\hline$\geq 650$ & $0.82_{-0.34}^{+0.45}$ & $0.80 \pm 0.25$ & $0.75 \pm 0.35$ & $0.04_{-0.04}^{+0.05}$ & $2.4_{-0.6}^{+0.7}$ & 2 \\
\hline \multicolumn{7}{|c|}{$N_{\mathrm{b}} \geq 2, m_{\mathrm{T}}^{\mathrm{b}} \geq 175 \mathrm{GeV}, N_{\mathrm{t}}=0, N_{\mathrm{res}}=0, N_{\mathrm{W}}=1$} \\
\hline $250-350$ & $56 \pm 9$ & $15 \pm 4$ & $5.7 \pm 1.7$ & $3.2 \pm 1.9$ & $80 \pm 13$ & 69 \\
\hline $350-450$ & $11 \pm 2$ & $8.0 \pm 2.3$ & $2.6 \pm 0.8$ & $2.6 \pm 1.8$ & $25 \pm 5$ & 29 \\
\hline $450-550$ & $1.8 \pm 0.6$ & $2.6 \pm 0.8$ & $1.0 \pm 0.4$ & $0.10 \pm 0.09$ & $5.5 \pm 1.2$ & 11 \\
\hline $550-650$ & $0.78 \pm 0.36$ & $0.80 \pm 0.34$ & $0.67 \pm 0.39$ & $<0.01$ & $2.3 \pm 0.7$ & 1 \\
\hline$\geq 650$ & $0.36_{-0.20}^{+0.25}$ & $1.1 \pm 0.4$ & $0.14 \pm 0.09$ & $0.02 \pm 0.02$ & $1.6 \pm 0.5$ & 1 \\
\hline \multicolumn{7}{|c|}{$N_{\mathrm{b}} \geq 2, m_{\mathrm{T}}^{\mathrm{b}} \geq 175 \mathrm{GeV}, N_{\mathrm{t}}=1, N_{\mathrm{res}}=0, N_{\mathrm{W}}=1$} \\
\hline$\geq 550$ & $0.21 \pm 0.14$ & $0.08 \pm 0.05$ & $0.10 \pm 0.03$ & $<0.01$ & $0.38 \pm 0.17$ & 1 \\
\hline \multicolumn{7}{|c|}{$N_{\mathrm{b}} \geq 2, m_{\mathrm{T}}^{\mathrm{b}} \geq 175 \mathrm{GeV}, N_{\mathrm{t}}=0, N_{\text {res }}=1, N_{\mathrm{W}}=1$} \\
\hline $250-350$ & $2.6 \pm 0.8$ & $0.51 \pm 0.23$ & $0.86 \pm 0.28$ & $0.05 \pm 0.06$ & $4.0 \pm 1.1$ & 5 \\
\hline $350-450$ & $0.60 \pm 0.29$ & $0.20 \pm 0.11$ & $0.51 \pm 0.19$ & $0.01 \pm 0.01$ & $1.3 \pm 0.4$ & 2 \\
\hline $450-550$ & $0.17 \pm 0.13$ & $0.14 \pm 0.08$ & $0.21 \pm 0.07$ & $<0.01$ & $0.52 \pm 0.20$ & 0 \\
\hline$\geq 550$ & $0.14 \pm 0.11$ & $0.07 \pm 0.06$ & $0.11 \pm 0.05$ & $<0.01$ & $0.32 \pm 0.14$ & 0 \\
\hline \multicolumn{7}{|c|}{$N_{\mathrm{b}} \geq 2, m_{\mathrm{T}}^{\mathrm{b}} \geq 175 \mathrm{GeV}, N_{\mathrm{t}}=1, N_{\mathrm{res}}=1, N_{\mathrm{W}}=0$} \\
\hline $250-350$ & $0.77 \pm 0.33$ & $<0.01$ & $0.25 \pm 0.15$ & $0.05 \pm 0.06$ & $1.1 \pm 0.4$ & 1 \\
\hline $350-450$ & $0.16 \pm 0.11$ & $<0.01$ & $0.17 \pm 0.06$ & $<0.01$ & $0.33 \pm 0.13$ & 1 \\
\hline$\geq 450$ & $0.01 \pm 0.01$ & $0.06 \pm 0.04$ & $0.20 \pm 0.08$ & $<0.01$ & $0.28 \pm 0.09$ & 0 \\
\hline \multicolumn{7}{|c|}{$N_{\mathrm{b}} \geq 2, m_{\mathrm{T}}^{\mathrm{b}} \geq 175 \mathrm{GeV}, N_{\mathrm{t}} \geq 2, N_{\text {res }}=0, N_{\mathrm{W}}=0$} \\
\hline$\geq 250$ & $0.06 \pm 0.06$ & $<0.01$ & $0.16 \pm 0.07$ & $<0.01$ & $0.22 \pm 0.10$ & 1 \\
\hline \multicolumn{7}{|c|}{$N_{\mathrm{b}} \geq 2, m_{\mathrm{T}}^{\mathrm{b}} \geq 175 \mathrm{GeV}, N_{\mathrm{t}}=0, N_{\mathrm{res}} \geq 2, N_{\mathrm{W}}=0$} \\
\hline$\geq 250$ & $1.9 \pm 0.8$ & $0.35 \pm 0.22$ & $1.5 \pm 0.7$ & $<0.01$ & $3.8 \pm 1.4$ & 3 \\
\hline \multicolumn{7}{|c|}{$N_{\mathrm{b}} \geq 2, m_{\mathrm{T}}^{\mathrm{b}} \geq 175 \mathrm{GeV}, N_{\mathrm{t}}=0, N_{\text {res }}=0, N_{\mathrm{W}} \geq 2$} \\
\hline$\geq 250$ & $1.5 \pm 0.7$ & $0.39 \pm 0.2$ & $0.17 \pm 0.13$ & $<0.01$ & $2.1 \pm 0.9$ & 3 \\
\hline
\end{tabular}

Table 6. Predicted background yields and the observation in different search regions for the high $\Delta m$ analysis. The total uncertainty is given for each background prediction. 

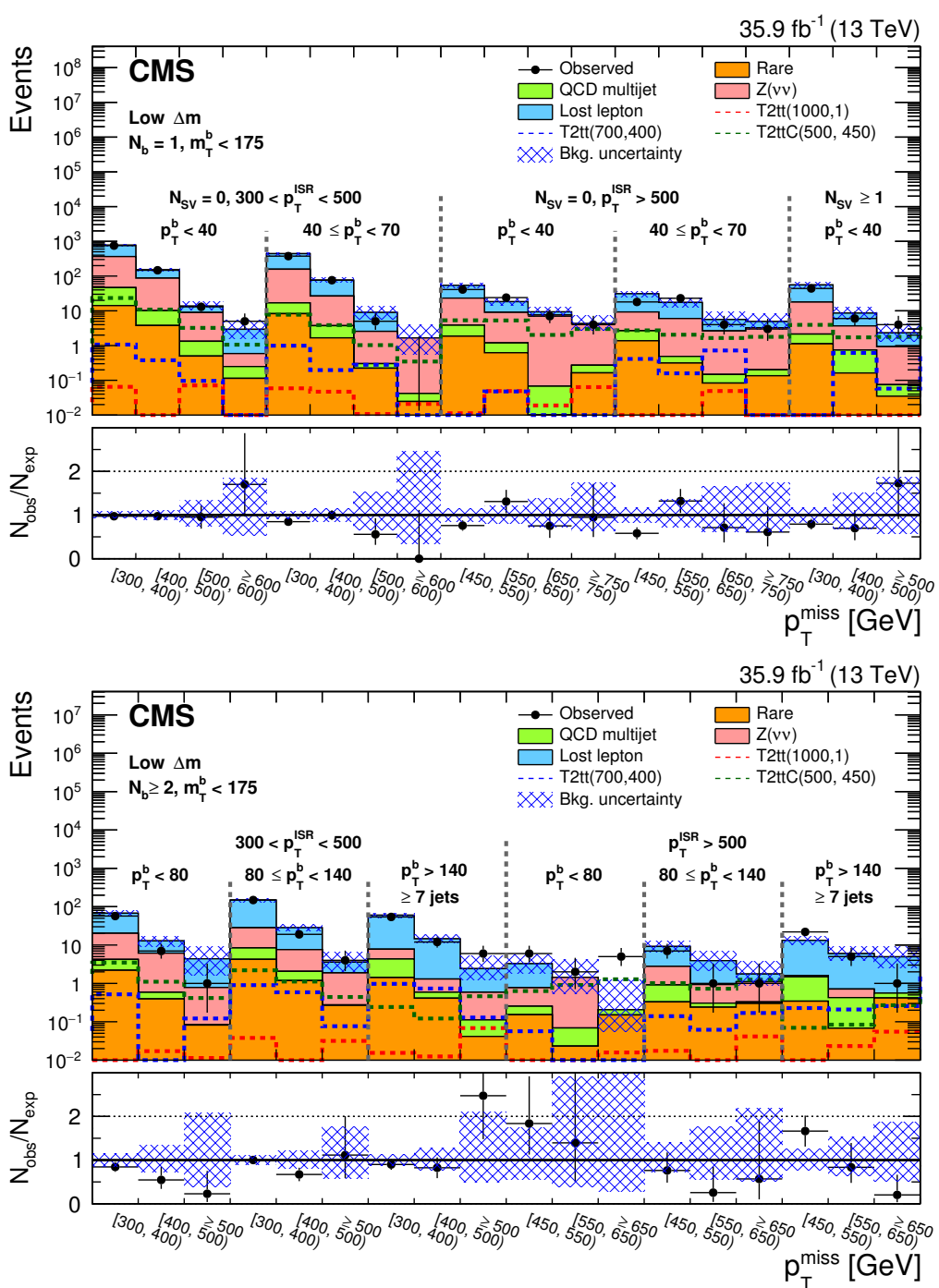

Figure 6. Observed data events and SM background predictions for the low $\Delta m$ search regions with $N_{\mathrm{b}}=1$ (upper), and $N_{\mathrm{b}} \geq 2$ (lower). Ratios of the observed to SM predicted event counts are shown in the lower panel of each plot. The shaded blue band represents the combined statistical and systematic uncertainties on the SM predictions. The one SR for which the result is not visible in the lower panel is discussed in the text. Units are GeV.

variable of little physical interest which however needs to be taken into account in order to have precise modeling of parameters that are of physical interest. Statistical uncertainties due to the limited number of simulated events are uncorrelated among all regions and backgrounds. The statistical uncertainties in background for different SR that are predicted using a common control region are assumed to be correlated.

The experimental uncertainties related to the isolated electron, muon, and $\tau_{\mathrm{h}}$ vetoes, $\mathrm{b}$ tagging, soft $\mathrm{b}$ tagging, jet energy scale, $p_{\mathrm{T}}^{\text {miss }}$ resolution, reweighting for pileup, and top quark and $\mathrm{W}$ boson tagging, are correlated across all SR and all backgrounds. The uncertainties in the lost lepton background estimate corresponding to differences in its t $\overline{\mathrm{t}}$ and $\mathrm{W}+$ jets fractions, or in the choice of $\mathrm{PDF}, \alpha_{S}$, and $\mu_{\mathrm{R}} / \mu_{\mathrm{F}}$ are also correlated for 

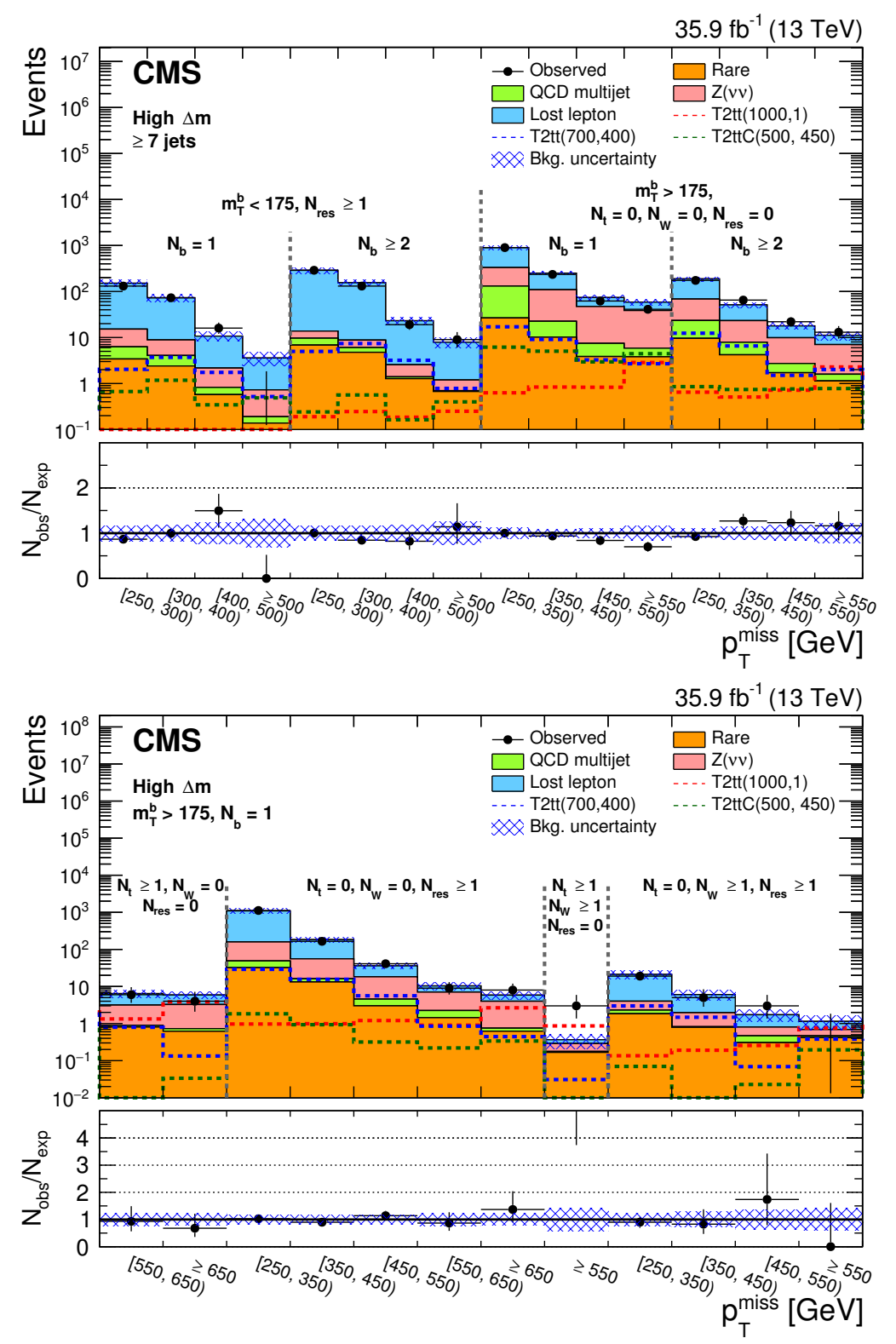

Figure 7. Observed data events and SM background predictions for the high $\Delta m$ search regions with $N_{\mathrm{j}} \geq 7$ (upper), and $m_{\mathrm{T}}^{\mathrm{b}}>175 \mathrm{GeV}$ and $N_{\mathrm{b}}=1$ (lower). Details of the selection applied is displayed on each plot. Ratios of the observed to SM predicted event counts are shown in the lower panel of each plot. The shaded blue band represents the statistical and systematic uncertainty on the prediction. The one SR for which the result is not visible in the lower panel is discussed in the text. Units are $\mathrm{GeV}$. 

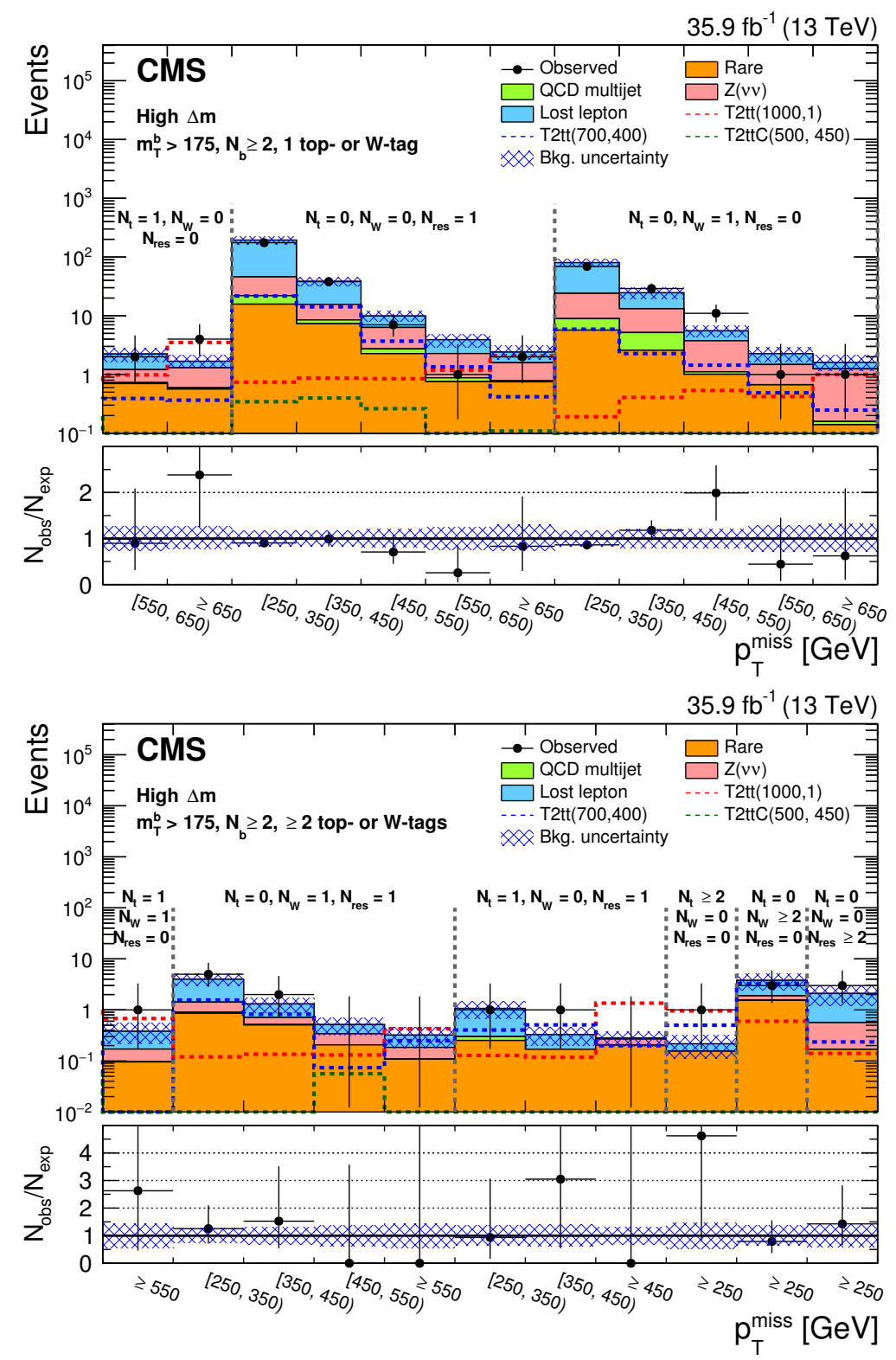

Figure 8. Observed data events and SM background predictions for the high $\Delta m$ search regions with one (upper), and at least two (lower) top quark or W boson candidates. Details of the selection applied is displayed on each plot. Ratios of the observed to SM predicted event counts are shown in the lower panel of each plot. The shaded blue band represents the statistical and systematic uncertainty on the prediction. Units are $\mathrm{GeV}$. 


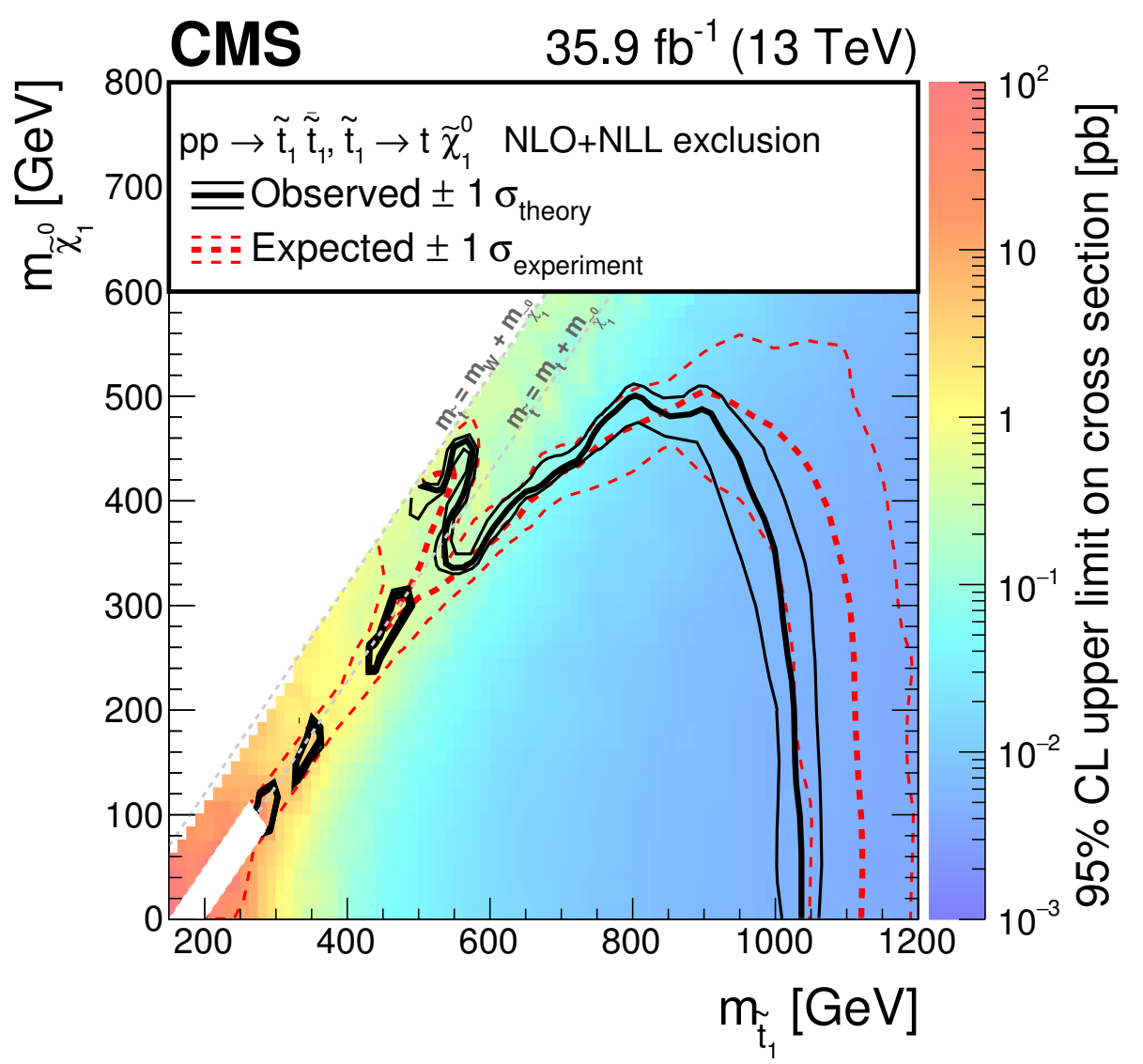

Figure 9. Exclusion limits at 95\% CL for top squark pair production followed by the decay $\widetilde{\mathrm{t}}_{1} \rightarrow \mathrm{t} \widetilde{\chi}_{1}^{0}(\mathrm{~T} 2 \mathrm{tt})$, in the mass plane $m_{\widetilde{\chi}_{1}^{0}}$ versus $m_{\widetilde{\mathrm{t}}_{1}}$. The areas to the left and below the solid black curves represent the observed exclusion and the \pm 1 standard deviation contours for the $\mathrm{NLO}+\mathrm{NLL}$ cross section calculations and their uncertainties [65]. The dashed red curves represent the corresponding expectation at $95 \% \mathrm{CL}$ and \pm 1 standard deviation contours for the associated experimental uncertainties. The "islands" represent regions that are not excluded by this search. The diagonal area where $\Delta m$ is very close to the top quark mass, corresponding to a very light $\widetilde{\chi}_{1}^{0}$, is

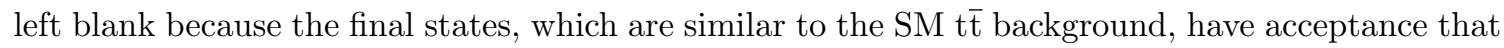
varies strongly with $\widetilde{\chi}_{1}^{0}$ mass, making it very difficult to model the signal acceptance in this region.

all SR and the single-lepton CR. Uncertainties due to the lepton correction factors are treated as anticorrelated between the single-lepton CR and the SR, since an underestimate of the efficiency for selecting leptons in the CR corresponds to an overestimate in the efficiency for vetoing leptons in the SR. The uncertainties assigned to the $\mathrm{Z}(\nu \nu)$ prediction are correlated separately to the uncertainties in $R_{Z}$ for all regions with the same $N_{\mathrm{b}}$ (and same $N_{\mathrm{SV}}$ ), and uncertainties originating from the discrepancies between the data-tosimulation ratios in $\mathrm{Z}(\ell \ell)+$ jets and $\gamma+$ jets events are correlated for all SR. The uncertainties in the QCD multijet background estimates corresponding to closure in the smearing method are correlated for all SR. For rare backgrounds, the uncertainties due to the variations of cross section, the PDF, $\alpha_{S}$, and $\mu_{\mathrm{R}} / \mu_{\mathrm{F}}$, are correlated for all SR. 


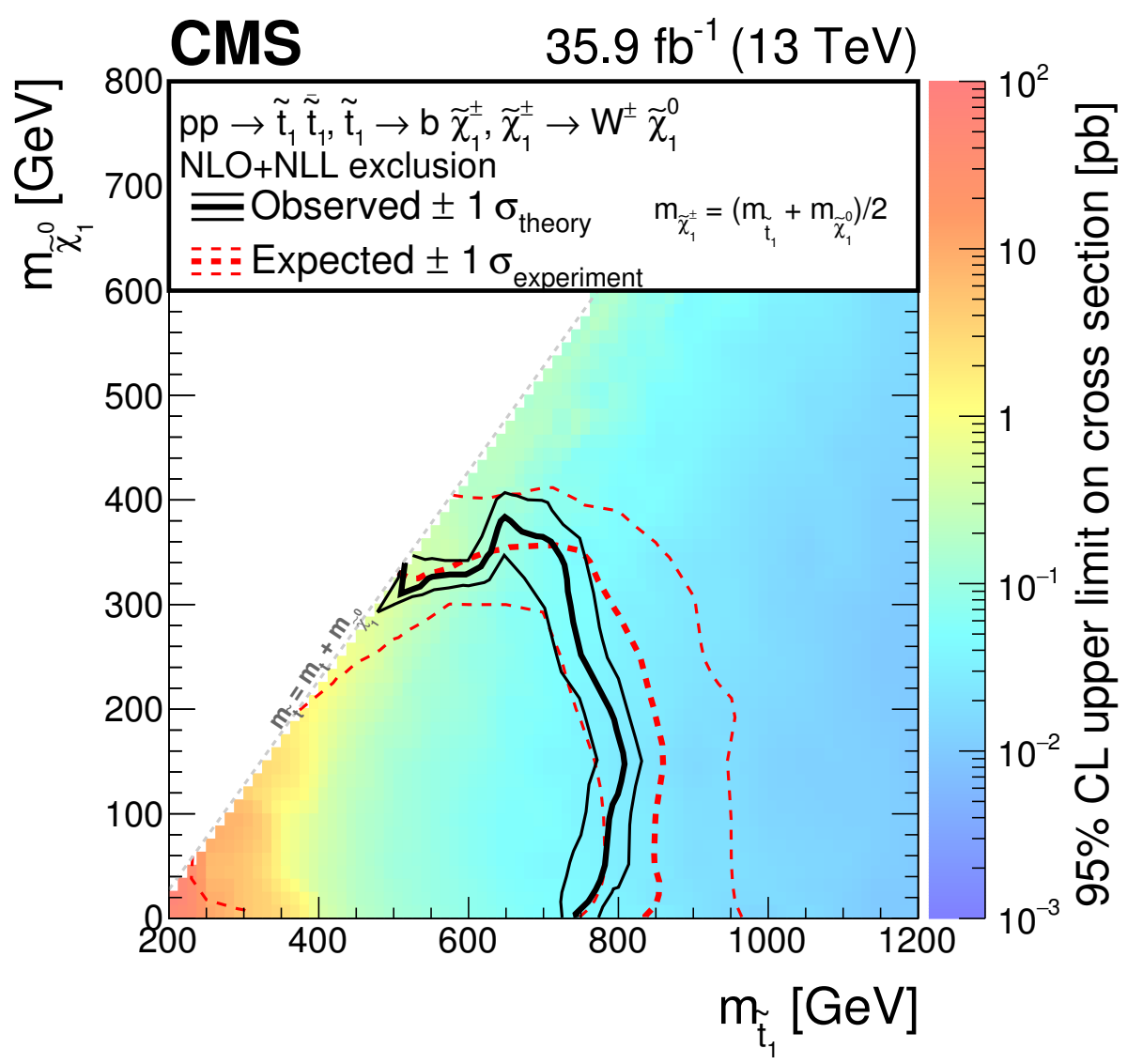

Figure 10. Exclusion limits at 95\% CL for top squark pair production followed by the decay $\widetilde{\mathrm{t}}_{1} \rightarrow \mathrm{b} \widetilde{\chi}_{1}^{ \pm} \rightarrow \mathrm{bW}^{ \pm} \widetilde{\chi}_{1}^{0}(\mathrm{~T} 2 \mathrm{bW})$, in the mass plane $m_{\widetilde{\chi}_{1}^{0}}$ versus $m_{\widetilde{\mathrm{t}}_{1}}$. The areas to the left and below the solid black curves represent the observed exclusion and the \pm 1 standard deviation contours for the NLO+NLL cross section calculations and their uncertainties [65]. The dashed red curves represent the corresponding expectation at $95 \% \mathrm{CL}$ and \pm 1 standard deviation contours for the associated experimental uncertainties. In the lower left corner of the diagram, where $\Delta m$ is close to the top quark mass, the sensitivity of the search is significantly reduced due to the fact that the $\widetilde{\mathrm{t}}_{1}$ decay products are soft and often escape detection.

For the simulated signal events, the differences between the fast simulation and the full Geant4-based simulation are taken into account. Appropriate corrections and uncertainties for differences observed in lepton selection efficiencies, b tagging performance, soft $\mathrm{b}$ tagging performance, jet energy scale, and top quark and $\mathrm{W}$ boson tagging efficiencies are applied to the predicted yields. The modeling of ISR plays an important role when it is relied upon to provide the $\widetilde{\mathrm{t}}_{1} \widetilde{\widetilde{\mathrm{t}}}_{1}$ system with a significant momentum boost, such as in modeling low $\Delta m$ signals. To improve on the modeling of the multiplicity of additional jets from ISR, the signal events are reweighted based on the number of ISR jets $\left(N_{\mathrm{j}}^{\mathrm{ISR}}\right)$ so as to make the jet multiplicity agree with data. The reweighting factors vary between 0.92 and 0.51 for $N_{\mathrm{j}}^{\mathrm{ISR}}$ between 1 and 6 . We take one half of the deviation from unity as the systematic uncertainty on these reweighting factors. We also assess uncertainties in the predicted signal 


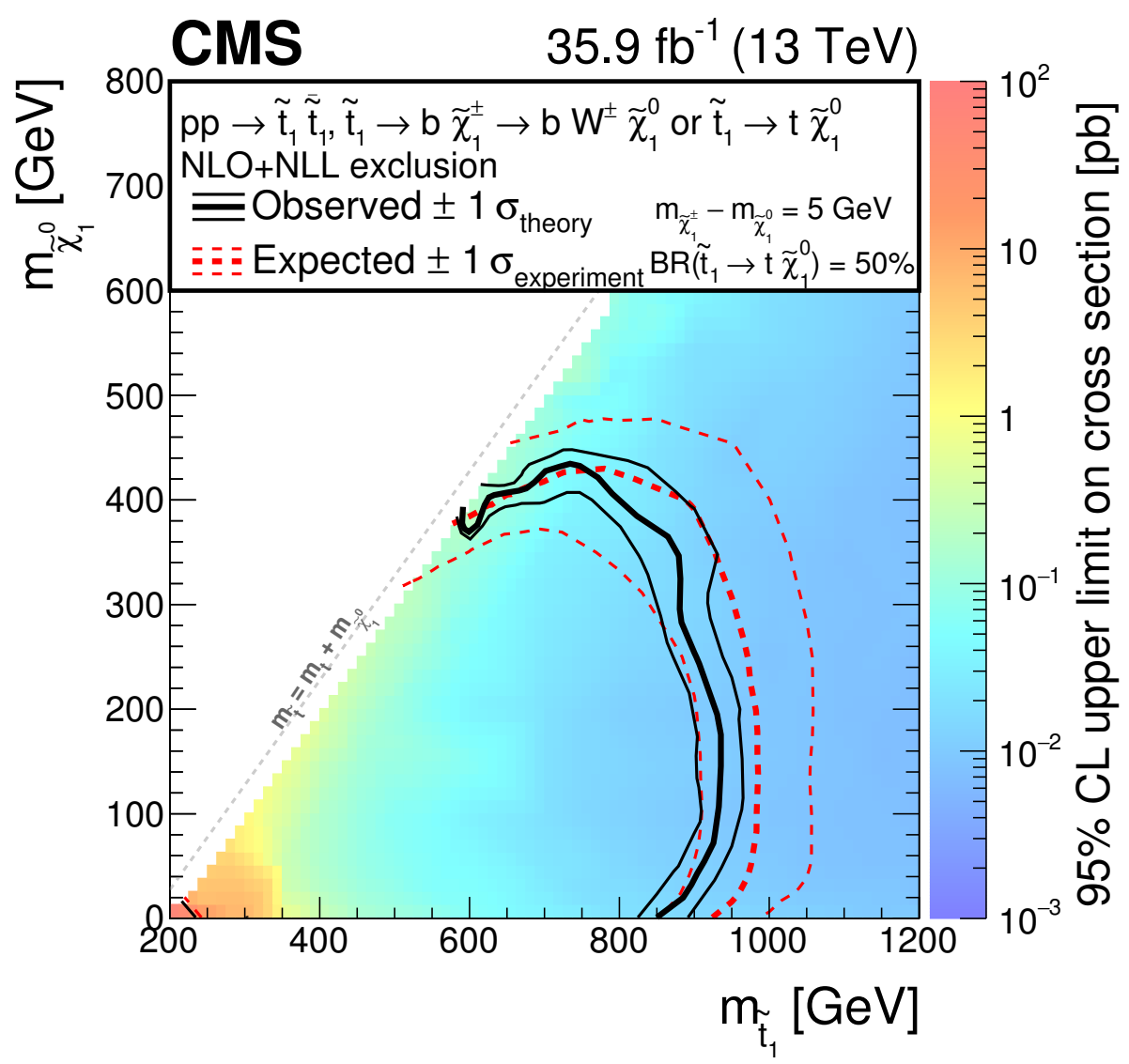

Figure 11. Exclusion limits at $95 \%$ CL for top squark pair production followed by the mixed decay $\mathrm{pp} \rightarrow \widetilde{\mathrm{t}}_{1} \overline{\mathrm{t}}_{1} \rightarrow \overline{\mathrm{t}} \widetilde{\chi}_{1}^{0} \mathrm{~b} \widetilde{\chi}_{1}^{+}(\mathrm{T} 2 \mathrm{tb})$ decay scenario, in the mass plane $m_{\widetilde{\chi}_{1}^{0}}$ versus $m_{\widetilde{\mathrm{t}}_{1}}$. In T2tb an assumption of a compressed mass spectrum in which the mass of $\tilde{\chi}_{1}^{ \pm}$is only $5 \mathrm{GeV}$ greater than that of $\widetilde{\chi}_{1}^{0}$, is considered. The areas to the left and below the solid black curves represent the observed exclusion and the \pm 1 standard deviation contours for the NLO+NLL cross section calculations and their uncertainties [65]. The dashed red curves represent the corresponding expectation at 95\% CL and \pm 1 standard deviation contours for the associated experimental uncertainties. In the lower left corner of the diagram, where $\Delta m$ is close to the top quark mass, the sensitivity of the search is significantly reduced due to the fact that the $\widetilde{t}_{1}$ decay products are soft and often escape detection.

arising from changes in $\mu_{\mathrm{R}}$ and $\mu_{\mathrm{F}}$. An extra correction and uncertainty is assigned for possible differences in $p_{\mathrm{T}}^{\text {miss }}$ resolution between the fast and the full GEANT4-based simulations. This uncertainty is correlated among all SR under the assumption of a uniform prior.

The results of the fit procedure are used to set exclusion limits in the models shown in figure 1. We set $95 \%$ CL exclusion limits for the large $\Delta m$ signal models, namely the T2tt, T2bW, and T2tb models described in section 1, in the mass plane $m_{\widetilde{\chi}_{1}^{0}}$ versus $m_{\widetilde{\mathrm{t}}_{1}}$, together with upper limits at 95\% CL on the signal cross section, under the hypotheses of the top squarks decaying as prescribed by these models. In the case of the T2tt model, figure 9, we can exclude $\widetilde{t}_{1}$ masses up to $1040 \mathrm{GeV}$ and $\widetilde{\chi}_{1}^{0}$ masses up to $500 \mathrm{GeV}$. The blank region below the diagonal in the lower left corner of the figure corresponds to values of $\Delta m$ that are very 


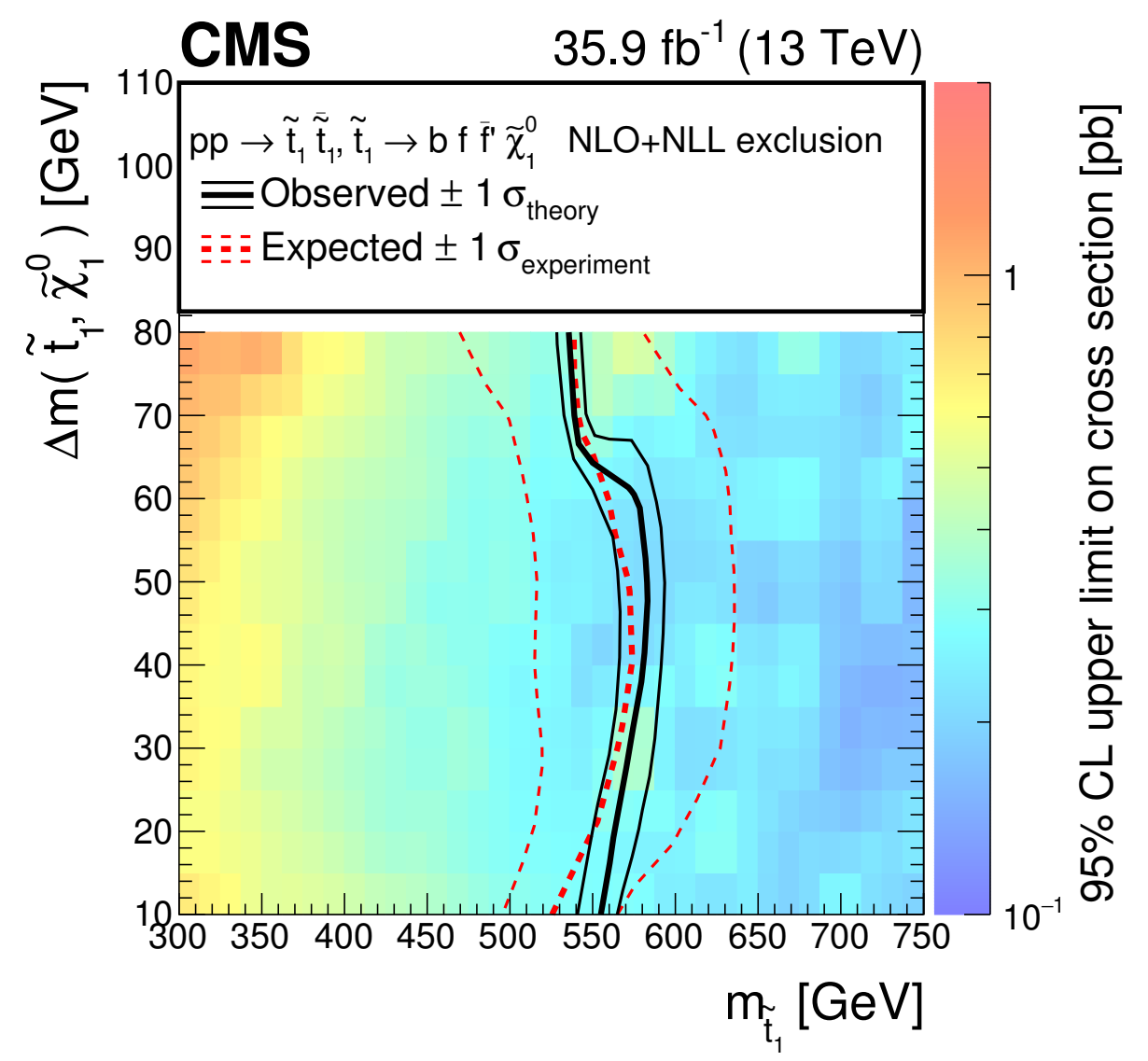

Figure 12. Exclusion limits at 95\% CL for top squark pair production followed by the four-body decay $\widetilde{\mathrm{t}}_{1} \rightarrow \mathrm{bf} \overline{f^{\prime}} \widetilde{\chi}_{1}^{0}(\mathrm{~T} 2 \mathrm{ttC})$ in the mass plane $\left.\Delta m \widetilde{\mathrm{t}}_{1}, \widetilde{\chi}_{1}^{0}\right)$ versus $m_{\widetilde{\mathrm{t}}_{1}}$. The areas to the left and below the solid black curves represent the observed exclusion and the \pm 1 standard deviation contours for the NLO+NLL cross section calculations and their uncertainties [65]. The dashed red curves represent the corresponding expectation at $95 \% \mathrm{CL}$ and \pm 1 standard deviation contours for the associated experimental uncertainties.

close to the top quark mass when the $\widetilde{\chi}_{1}^{0}$ is very light. This area is particularly challenging due to the similarity of the final states to SM tE background, which results in a significant contamination from leptonic signal events in the control regions. Our analysis does not have adequate signal discrimination to provide exclusion limits in this region. Figure 10 shows the exclusion limits obtained for the T2bW model. Under this decay hypothesis, for which the $\widetilde{\chi}_{1}^{ \pm}$mass lies halfway between the $\widetilde{t}_{1}$ and $\widetilde{\chi}_{1}^{0}$ masses, we can exclude $\widetilde{t}_{1}$ masses up to $800 \mathrm{GeV}$ and $\widetilde{\chi}_{1}^{0}$ masses up to $360 \mathrm{GeV}$. Figure 11 addresses the T2tb model, in which both of these decay modes are allowed with equal probability while also assuming a compressed mass spectrum in which the mass of the $\widetilde{\chi}_{1}^{ \pm}$is only $5 \mathrm{GeV}$ greater than that of the $\widetilde{\chi}_{1}^{0}$. We can exclude for this model $\widetilde{\mathrm{t}}_{1}$ masses up to $940 \mathrm{GeV}$, and $\widetilde{\chi}_{1}^{0}$ masses up to $440 \mathrm{GeV}$.

We also set exclusion limits in the mass plane $\Delta m$ versus $m_{\tilde{t}_{1}}$ for small $\Delta m$ signal models in which the mass difference between the $\widetilde{\mathrm{t}}_{1}$ and $\widetilde{\chi}_{1}^{0}$ is smaller than the $\mathrm{W}$ boson mass, namely the T2ttC, T2bWC, and T2cc models described in section 1 . Note that for 


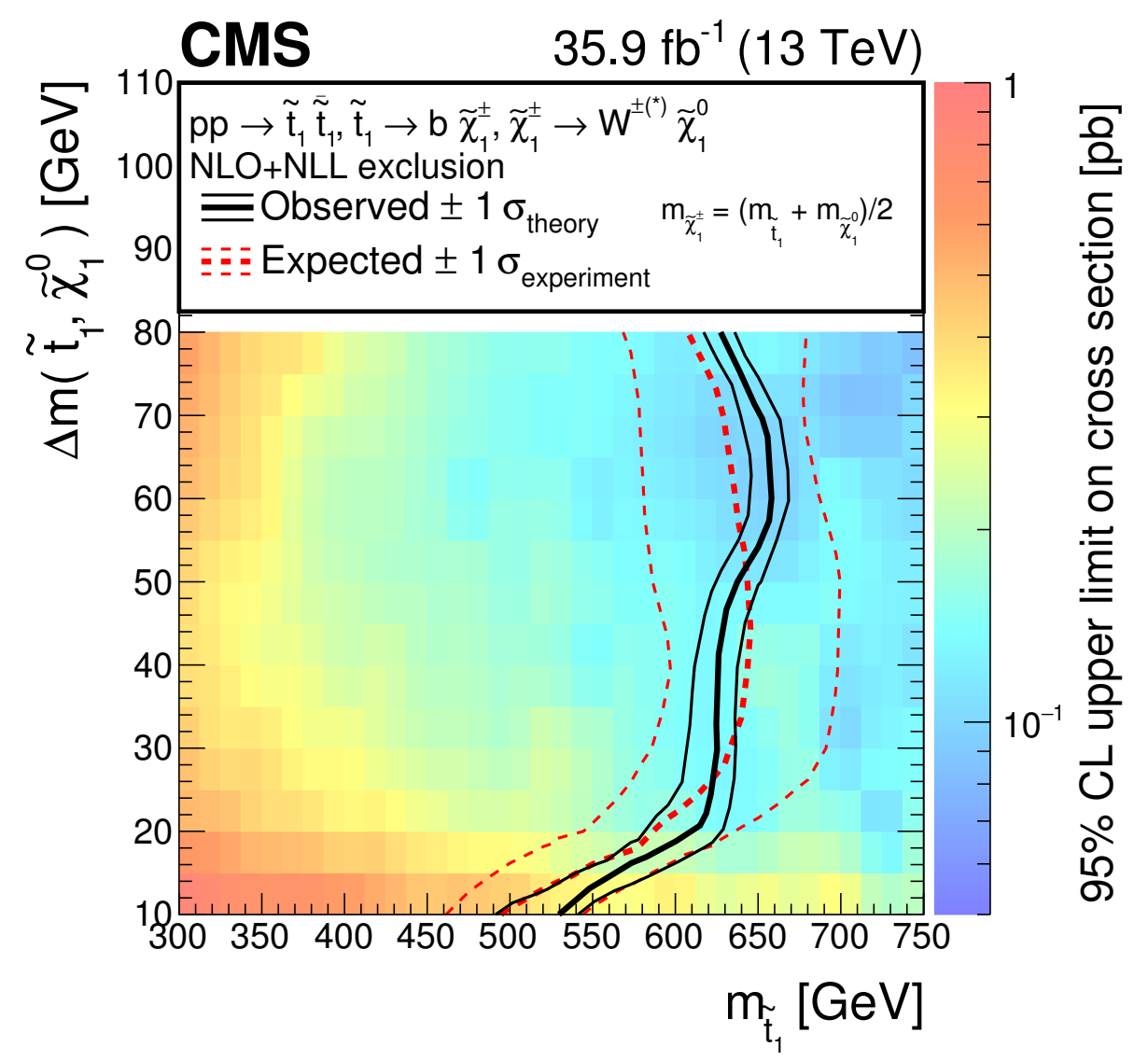

Figure 13. Exclusion limits at 95\% CL for top squark pair production followed by the decay $\widetilde{\mathrm{t}}_{1} \rightarrow \mathrm{b} \widetilde{\chi}_{1}^{ \pm} \rightarrow \mathrm{bf} \bar{f}^{\prime} \widetilde{\chi}_{1}^{0}(\mathrm{~T} 2 \mathrm{bWC})$ in the mass plane $\Delta m\left(\widetilde{\mathrm{t}}_{1}, \widetilde{\chi}_{1}^{0}\right)$ versus $m_{\widetilde{\mathrm{t}}_{1}}$. The areas to the left and below the solid black curves represent the observed exclusion and the \pm 1 standard deviation contours for the NLO+NLL cross section calculations and their uncertainties [65]. The dashed red curves represent the corresponding expectation at $95 \% \mathrm{CL}$ and \pm 1 standard deviation contours for the associated experimental uncertainties.

mass configurations with $\Delta m<30 \mathrm{GeV}$, the $\widetilde{\mathrm{t}}_{1}$ lifetime becomes significant [107]. This is not taken into account in the simulation that we used for the T2ttC model. We therefore also consider the T2bWC model, which ensures reasonable lifetimes for the $\widetilde{t}_{1}$ in this region where the $\widetilde{\chi}_{1}^{ \pm}$decays to an off-shell $\mathrm{W}$ boson and an $\widetilde{\chi}_{1}^{0}$. Figures 12 and 13 show the exclusion limits obtained for exclusive T2ttC and exclusive T2bWC decays, respectively. We can exclude $\widetilde{t}_{1}$ masses up to $580(660) \mathrm{GeV}$, and $\widetilde{\chi}_{1}^{0}$ masses up to 540 (610) GeV for the T2ttC (T2bWC) model. Finally, figure 14 shows the exclusion limits obtained for the T2cc model in which we exclude $\widetilde{t}_{1}$ and $\widetilde{\chi}_{1}^{0}$ masses up to 560 and $520 \mathrm{GeV}$, respectively.

\section{Summary}

A search is presented for direct top squark pair production in the all-jets final states based upon data collected with the CMS detector in pp collisions at a center-of-mass energy of 


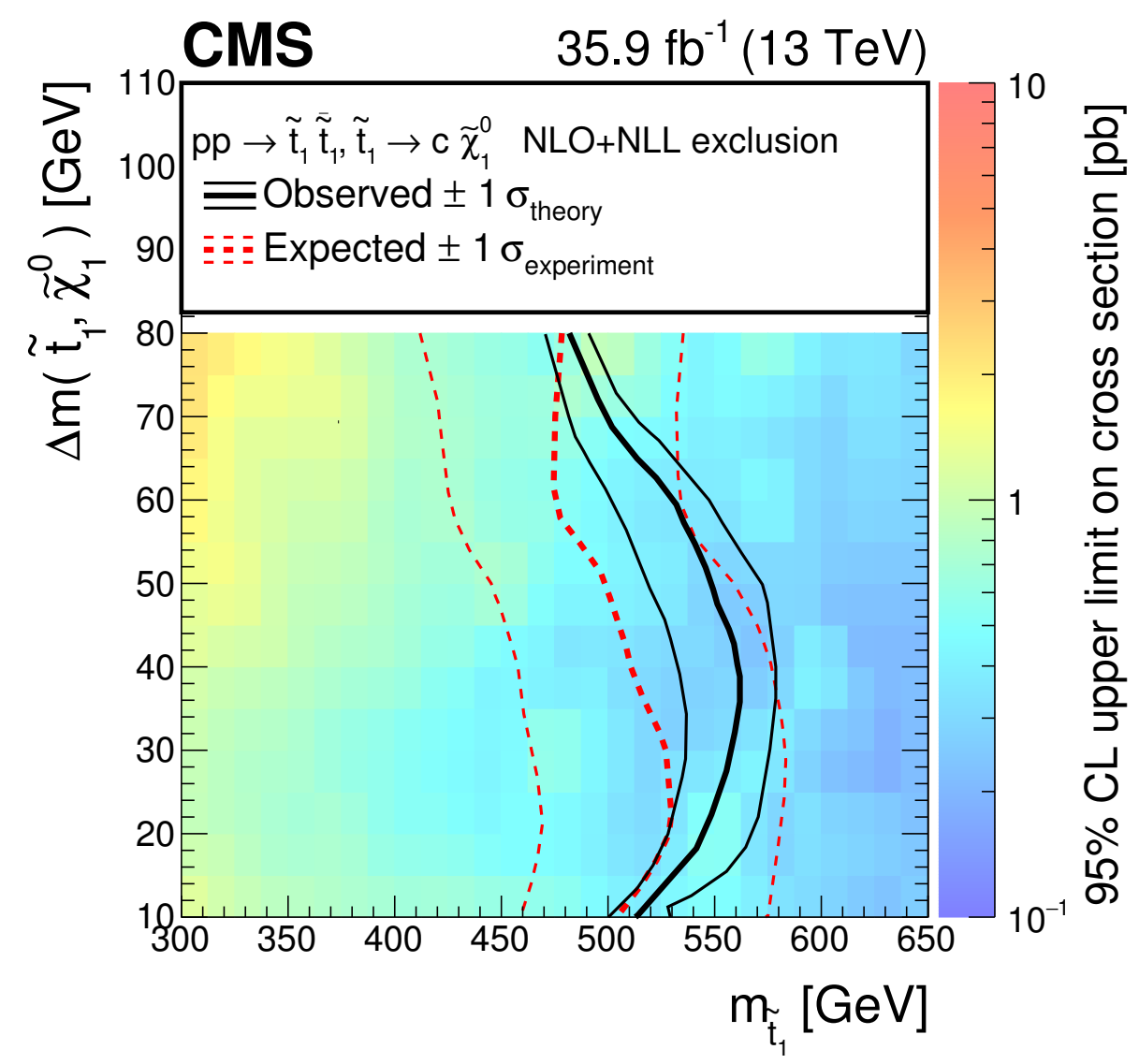

Figure 14. Exclusion limits at 95\% CL for top squark pair production followed by the decay $\widetilde{\mathrm{t}}_{1} \rightarrow \mathrm{c} \widetilde{\chi}_{1}^{0}(\mathrm{~T} 2 \mathrm{cc})$ in the mass plane $\Delta m\left(\widetilde{\mathrm{t}}_{1}, \widetilde{\chi}_{1}^{0}\right)$ versus $m_{\widetilde{\mathrm{t}}_{1}}$. The areas to the left and below the solid black curves represent the observed exclusion and the \pm 1 standard deviation contours for the NLO+NLL cross section calculations and their uncertainties [65]. The dashed red curves represent the corresponding expectation at $95 \% \mathrm{CL}$ and \pm 1 standard deviation contours for the associated experimental uncertainties.

$13 \mathrm{TeV}$, corresponding to an integrated luminosity of $35.9 \mathrm{fb}^{-1}$. The search is optimized for discovery through a variety of signatures. No significant excess of events is observed beyond the expected contribution from SM processes, and exclusion limits are set at a $95 \%$ confidence level in the context of simplified models [48-50] of direct top squark pair production.

In the parameter space of large mass differences between the $\widetilde{t}_{1}$ and $\widetilde{\chi}_{1}^{0}$ that permit the $\widetilde{\mathrm{t}}_{1}$ to decay to an on-shell top quark and a neutralino, top squark masses up to $1040 \mathrm{GeV}$ and $\widetilde{\chi}_{1}^{0}$ masses up to $500 \mathrm{GeV}$ are excluded. Alternatively, when the top squark decays to a bottom quark and a $\widetilde{\chi}_{1}^{ \pm}, \widetilde{t}_{1}$ masses up to $800 \mathrm{GeV}$ and $\widetilde{\chi}_{1}^{0}$ masses up to $360 \mathrm{GeV}$ are excluded. Finally, for possibilities in which the branching fractions for these two top squark decay modes equal $50 \%$, including the assumption of a compressed mass spectrum with the mass of the $\widetilde{\chi}_{1}^{ \pm}$only $5 \mathrm{GeV}$ greater than that of $\widetilde{\chi}_{1}^{0}$, top squark masses up to $940 \mathrm{GeV}$ and $\widetilde{\chi}_{1}^{0}$ masses up to $440 \mathrm{GeV}$ are excluded. 
In the regions of parameter space where the mass difference between the $\widetilde{t}_{1}$ and $\widetilde{\chi}_{1}^{0}$ is smaller than the mass of the $\mathrm{W}$ boson, we consider four-body decays of top squarks in which top squark masses up to $580 \mathrm{GeV}$ are excluded for a neutralino mass of $540 \mathrm{GeV}$. An additional decay that is relevant in this parameter space is one in which the top squark decays to a bottom quark and a $\widetilde{\chi}_{1}^{ \pm}$, that then decays to a virtual $\mathrm{W}$ boson and a $\widetilde{\chi}_{1}^{0}$. Here, top squark masses up to $660 \mathrm{GeV}$ are excluded for a neutralino mass of $610 \mathrm{GeV}$. Finally, we consider decays through a flavor changing neutral current process where the $\tilde{\mathrm{t}}_{1}$ decays to a c quark and a $\widetilde{\chi}_{1}^{0}$. In this case, $\widetilde{\mathrm{t}}_{1}$ and $\widetilde{\chi}_{1}^{0}$ masses up to $560 \mathrm{GeV}$ and up to $520 \mathrm{GeV}$, respectively, are excluded.

In summary, we present a search that takes advantage of a large new set of data collected by the CMS experiment in 2016, as well as a variety of new methods that yield exclusion limits for a wide array of top squark decay modes in planes of $m_{\widetilde{\chi}_{1}^{0}}$ versus $m_{\widetilde{\mathfrak{t}}_{1}}$ and $m_{\widetilde{\mathrm{t}}_{1}}-m_{\widetilde{\chi}_{1}^{0}}$ versus $m_{\widetilde{\mathrm{t}}_{1}}$ that extend significantly beyond those obtained in previous searches.

\section{Acknowledgments}

We congratulate our colleagues in the CERN accelerator departments for the excellent performance of the LHC and thank the technical and administrative staffs at CERN and at other CMS institutes for their contributions to the success of the CMS effort. In addition, we gratefully acknowledge the computing centers and personnel of the Worldwide LHC Computing Grid for delivering so effectively the computing infrastructure essential to our analyses. Finally, we acknowledge the enduring support for the construction and operation of the LHC and the CMS detector provided by the following funding agencies: BMWFW and FWF (Austria); FNRS and FWO (Belgium); CNPq, CAPES, FAPERJ, and FAPESP (Brazil); MES (Bulgaria); CERN; CAS, MoST, and NSFC (China); COLCIENCIAS (Colombia); MSES and CSF (Croatia); RPF (Cyprus); SENESCYT (Ecuador); MoER, ERC IUT, and ERDF (Estonia); Academy of Finland, MEC, and HIP (Finland); CEA and CNRS/IN2P3 (France); BMBF, DFG, and HGF (Germany); GSRT (Greece); OTKA and NIH (Hungary); DAE and DST (India); IPM (Iran); SFI (Ireland); INFN (Italy); MSIP and NRF (Republic of Korea); LAS (Lithuania); MOE and UM (Malaysia); BUAP, CINVESTAV, CONACYT, LNS, SEP, and UASLP-FAI (Mexico); MBIE (New Zealand); PAEC (Pakistan); MSHE and NSC (Poland); FCT (Portugal); JINR (Dubna); MON, RosAtom, RAS, RFBR and RAEP (Russia); MESTD (Serbia); SEIDI, CPAN, PCTI and FEDER (Spain); Swiss Funding Agencies (Switzerland); MST (Taipei); ThEPCenter, IPST, STAR, and NSTDA (Thailand); TUBITAK and TAEK (Turkey); NASU and SFFR (Ukraine); STFC (United Kingdom); DOE and NSF (U.S.A.).

Individuals have received support from the Marie-Curie program and the European Research Council and Horizon 2020 Grant, contract No. 675440 (European Union); the Leventis Foundation; the A. P. Sloan Foundation; the Alexander von Humboldt Foundation; the Belgian Federal Science Policy Office; the Fonds pour la Formation à la Recherche dans l'Industrie et dans l'Agriculture (FRIA-Belgium); the Agentschap voor Innovatie door Wetenschap en Technologie (IWT-Belgium); the Ministry of Education, Youth and Sports (MEYS) of the Czech Republic; the Council of Science and Industrial Research, 
India; the HOMING PLUS program of the Foundation for Polish Science, cofinanced from European Union, Regional Development Fund, the Mobility Plus program of the Ministry of Science and Higher Education, the National Science Center (Poland), contracts Harmonia 2014/14/M/ST2/00428, Opus 2014/13/B/ST2/02543, 2014/15/B/ST2/03998, and 2015/19/B/ST2/02861, Sonata-bis 2012/07/E/ST2/01406; the National Priorities Research Program by Qatar National Research Fund; the Programa Clarín-COFUND del Principado de Asturias; the Thalis and Aristeia programs cofinanced by EU-ESF and the Greek NSRF; the Rachadapisek Sompot Fund for Postdoctoral Fellowship, Chulalongkorn University and the Chulalongkorn Academic into Its 2nd Century Project Advancement Project (Thailand); and the Welch Foundation, contract C-1845.

Open Access. This article is distributed under the terms of the Creative Commons Attribution License (CC-BY 4.0), which permits any use, distribution and reproduction in any medium, provided the original author(s) and source are credited.

\section{References}

[1] ATLAS collaboration, Observation of a new particle in the search for the Standard Model Higgs boson with the ATLAS detector at the LHC, Phys. Lett. B 716 (2012) 1 [arXiv:1207.7214] [INSPIRE].

[2] CMS collaboration, Observation of a new boson at a mass of $125 \mathrm{GeV}$ with the CMS experiment at the LHC, Phys. Lett. B 716 (2012) 30 [arXiv:1207.7235] [INSPIRE].

[3] CMS collaboration, Observation of a new boson with mass near $125 \mathrm{GeV}$ in pp collisions at $\sqrt{s}=7$ and $8 \mathrm{TeV}$, JHEP 06 (2013) 081 [arXiv: 1303.4571] [INSPIRE].

[4] ATLAS collaboration, Measurement of the Higgs boson mass from the $H \rightarrow \gamma \gamma$ and $H \rightarrow Z Z^{*} \rightarrow 4 \ell$ channels with the ATLAS detector using $25 \mathrm{fb}^{-1}$ of pp collision data, Phys. Rev. D 90 (2014) 052004 [arXiv: 1406.3827] [INSPIRE].

[5] CMS collaboration, Precise determination of the mass of the Higgs boson and tests of compatibility of its couplings with the standard model predictions using proton collisions at 7 and 8 TeV, Eur. Phys. J. C 75 (2015) 212 [arXiv:1412.8662] [INSPIRE].

[6] ATLAS and CMS collaborations, Combined measurement of the Higgs boson mass in pp collisions at $\sqrt{s}=7$ and 8 TeV with the ATLAS and CMS experiments, Phys. Rev. Lett. 114 (2015) 191803 [arXiv: 1503.07589] [INSPIRE].

[7] K.G. Wilson, The renormalization group and strong interactions, Phys. Rev. D 3 (1971) 1818 [INSPIRE].

[8] G.F. Giudice, Naturalness after LHC8, PoS(EPS-HEP 2013) 163 [arXiv:1307.7879] [INSPIRE].

[9] G. 't Hooft, Naturalness, chiral symmetry, and spontaneous chiral symmetry breaking, NATO Sci. Ser. B 59 (1980) 135 [INSPIRE].

[10] E. Witten, Dynamical breaking of supersymmetry, Nucl. Phys. B 188 (1981) 513 [InSPIRE].

[11] M. Dine, W. Fischler and M. Srednicki, Supersymmetric technicolor, Nucl. Phys. B 189 (1981) 575 [INSPIRE].

[12] S. Dimopoulos and S. Raby, Supercolor, Nucl. Phys. B 192 (1981) 353 [INSPIRE]. 
[13] S. Dimopoulos and H. Georgi, Softly broken supersymmetry and SU(5), Nucl. Phys. B 193 (1981) 150 [INSPIRE].

[14] R.K. Kaul and P. Majumdar, Cancellation of quadratically divergent mass corrections in globally supersymmetric spontaneously broken gauge theories, Nucl. Phys. B 199 (1982) 36 [INSPIRE].

[15] F. Zwicky, Die Rotverschiebung von extragalaktischen Nebeln (in German), Helv. Phys. Acta 6 (1933) 110 [Gen. Relativ. Gravit. 41 (2009) 207] [InSPIRE].

[16] V.C. Rubin and W.K. Ford, Jr., Rotation of the Andromeda nebula from a spectroscopic survey of emission regions, Astrophys. J. 159 (1970) 379 [INSPIRE].

[17] Particle Data Group collaboration, C. Patrignani et al., Review of particle physics, Chin. Phys. C 40 (2016) 100001 [InSPIRE].

[18] R. Barbieri and G.F. Giudice, Upper bounds on supersymmetric particle masses, Nucl. Phys. B 306 (1988) 63 [inSPIRE].

[19] B. de Carlos and J.A. Casas, One loop analysis of the electroweak breaking in supersymmetric models and the fine tuning problem, Phys. Lett. B 309 (1993) 320 [hep-ph/9303291] [INSPIRE].

[20] S. Dimopoulos and G.F. Giudice, Naturalness constraints in supersymmetric theories with nonuniversal soft terms, Phys. Lett. B 357 (1995) 573 [hep-ph/9507282] [INSPIRE].

[21] R. Barbieri, G.R. Dvali and L.J. Hall, Predictions from a U(2) flavor symmetry in supersymmetric theories, Phys. Lett. B 377 (1996) 76 [hep-ph/9512388] [INSPIRE].

[22] J.L. Feng, Naturalness and the status of supersymmetry, Ann. Rev. Nucl. Part. Sci. 63 (2013) 351 [arXiv: 1302.6587] [INSPIRE].

[23] L. Evans and P. Bryant, LHC machine, 2008 JINST 3 S08001 [INSPIRE].

[24] J. Wess and B. Zumino, Supergauge transformations in four-dimensions, Nucl. Phys. B 70 (1974) 39 [INSPIRE].

[25] G.R. Farrar and P. Fayet, Phenomenology of the production, decay and detection of new hadronic states associated with supersymmetry, Phys. Lett. 76B (1978) 575 [INSPIRE].

[26] J.L. Feng, Dark matter candidates from particle physics and methods of detection, Ann. Rev. Astron. Astrophys. 48 (2010) 495 [arXiv: 1003.0904] [INSPIRE].

[27] ATLAS collaboration, Search for a supersymmetric partner to the top quark in final states with jets and missing transverse momentum at $\sqrt{s}=7 \mathrm{TeV}$ with the ATLAS detector, Phys. Rev. Lett. 109 (2012) 211802 [arXiv:1208.1447] [INSPIRE].

[28] ATLAS collaboration, Search for direct top squark pair production in final states with one isolated lepton, jets and missing transverse momentum in $\sqrt{s}=7 \mathrm{TeV}$ pp collisions using $4.7 \mathrm{fb}^{-1}$ of ATLAS data, Phys. Rev. Lett. 109 (2012) 211803 [arXiv:1208.2590] [InSPIRE].

[29] ATLAS collaboration, Search for a heavy top-quark partner in final states with two leptons with the ATLAS detector at the LHC, JHEP 11 (2012) 094 [arXiv: 1209.4186] [INSPIRE].

[30] ATLAS collaboration, Search for direct top-squark pair production in final states with two leptons in pp collisions at $\sqrt{s}=8 \mathrm{TeV}$ with the ATLAS detector, JHEP 06 (2014) 124 [arXiv: 1403.4853] [INSPIRE]. 
[31] ATLAS collaboration, Search for direct third-generation squark pair production in final states with missing transverse momentum and two b-jets in $\sqrt{s}=8 \mathrm{TeV}$ pp collisions with the ATLAS detector, JHEP 10 (2013) 189 [arXiv:1308.2631] [INSPIRE].

[32] ATLAS collaboration, Measurement of spin correlation in top-antitop quark events and search for top squark pair production in pp collisions at $\sqrt{s}=8 \mathrm{TeV}$ using the ATLAS detector, Phys. Rev. Lett. 114 (2015) 142001 [arXiv:1412.4742] [INSPIRE].

[33] ATLAS collaboration, Search for pair-produced third-generation squarks decaying via charm quarks or in compressed supersymmetric scenarios in pp collisions at $\sqrt{s}=8 \mathrm{TeV}$ with the ATLAS detector, Phys. Rev. D 90 (2014) 052008 [arXiv:1407.0608] [INSPIRE].

[34] ATLAS collaboration, ATLAS run 1 searches for direct pair production of third-generation squarks at the Large Hadron Collider, Eur. Phys. J. C 75 (2015) 510 [Erratum ibid. C 76 (2016) 153] [arXiv:1506.08616] [INSPIRE].

[35] CMS collaboration, Search for top-squark pair production in the single-lepton final state in pp collisions at $\sqrt{s}=8 \mathrm{TeV}$, Eur. Phys. J. C 73 (2013) 2677 [arXiv:1308.1586] [INSPIRE].

[36] CMS collaboration, Search for supersymmetry in hadronic final states with missing transverse energy using the variables $\alpha_{T}$ and b-quark multiplicity in pp collisions at $\sqrt{s}=8$ TeV, Eur. Phys. J. C 73 (2013) 2568 [arXiv:1303.2985] [InSPIRE].

[37] CMS collaboration, Search for supersymmetry using razor variables in events with b-tagged jets in pp collisions at $\sqrt{s}=8$ TeV, Phys. Rev. D 91 (2015) 052018 [arXiv:1502.00300] [INSPIRE].

[38] CMS collaboration, Searches for third-generation squark production in fully hadronic final states in proton-proton collisions at $\sqrt{s}=8 \mathrm{TeV}$, JHEP 06 (2015) 116 [arXiv:1503.08037] [INSPIRE].

[39] CMS collaboration, Search for direct pair production of supersymmetric top quarks decaying to all-hadronic final states in pp collisions at $\sqrt{s}=8$ TeV, Eur. Phys. J. C 76 (2016) 460 [arXiv: 1603.00765] [INSPIRE].

[40] ATLAS collaboration, Search for top squarks in final states with one isolated lepton, jets and missing transverse momentum in $\sqrt{s}=13 \mathrm{TeV}$ pp collisions with the ATLAS detector, Phys. Rev. D 94 (2016) 052009 [arXiv: 1606.03903] [InSPIRE].

[41] CMS collaboration, Search for new physics with the $M_{\mathrm{T} 2}$ variable in all-jets final states produced in pp collisions at $\sqrt{s}=13 \mathrm{TeV}$, JHEP 10 (2016) 006 [arXiv:1603.04053] [INSPIRE].

[42] CMS collaboration, Inclusive search for supersymmetry using razor variables in pp collisions at $\sqrt{s}=13 \mathrm{TeV}$, Phys. Rev. D 95 (2017) 012003 [arXiv:1609.07658] [InSPIRE].

[43] CMS collaboration, A search for new phenomena in pp collisions at $\sqrt{s}=13 \mathrm{TeV}$ in final states with missing transverse momentum and at least one jet using the $\alpha_{\mathrm{T}}$ variable, Eur. Phys. J. C 77 (2017) 294 [arXiv: 1611.00338] [INSPIRE].

[44] CMS collaboration, Searches for pair production of third-generation squarks in $\sqrt{s}=13 \mathrm{TeV}$ pp collisions, Eur. Phys. J. C 77 (2017) 327 [arXiv:1612.03877] [InSPIRE].

[45] ATLAS collaboration, Search for direct top squark pair production in events with a Higgs or $Z$ boson and missing transverse momentum in $\sqrt{s}=13 \mathrm{TeV} p p$ collisions with the ATLAS detector, JHEP 08 (2017) 006 [arXiv:1706.03986] [INSPIRE]. 
[46] CMS collaboration, Search for supersymmetry in multijet events with missing transverse momentum in proton-proton collisions at 13 TeV, Phys. Rev. D 96 (2017) 032003 [arXiv:1704.07781] [INSPIRE].

[47] CMS collaboration, Search for new phenomena with the $M_{\mathrm{T} 2}$ variable in the all-hadronic final state produced in proton-proton collisions at $\sqrt{s}=13 \mathrm{TeV}$, arXiv:1705.04650 [INSPIRE].

[48] J. Alwall, P. Schuster and N. Toro, Simplified models for a first characterization of new physics at the LHC, Phys. Rev. D 79 (2009) 075020 [arXiv:0810.3921] [InSPIRE].

[49] J. Alwall, M.-P. Le, M. Lisanti and J.G. Wacker, Model-independent jets plus missing energy searches, Phys. Rev. D 79 (2009) 015005 [arXiv:0809.3264] [InSPIRE].

[50] LHC New Physics Working Group collaboration, D. Alves, Simplified models for LHC new physics searches, J. Phys. G 39 (2012) 105005 [arXiv:1105.2838] [InSPIRE].

[51] C. Balázs, M. Carena and C.E.M. Wagner, Dark matter, light stops and electroweak baryogenesis, Phys. Rev. D 70 (2004) 015007 [hep-ph/0403224] [INSPIRE].

[52] CMS collaboration, The CMS experiment at the CERN LHC, 2008 JINST 3 S08004 [INSPIRE].

[53] J. Alwall et al., The automated computation of tree-level and next-to-leading order differential cross sections and their matching to parton shower simulations, JHEP $\mathbf{0 7}$ (2014) 079 [arXiv: 1405.0301] [INSPIRE].

[54] NNPDF collaboration, R.D. Ball et al., Parton distributions for the LHC run II, JHEP 04 (2015) 040 [arXiv: 1410.8849] [INSPIRE].

[55] P. Nason, A new method for combining NLO QCD with shower Monte Carlo algorithms, JHEP 11 (2004) 040 [hep-ph/0409146] [INSPIRE].

[56] S. Frixione, P. Nason and C. Oleari, Matching NLO QCD computations with parton shower simulations: the POWHEG method, JHEP 11 (2007) 070 [arXiv:0709.2092] [INSPIRE].

[57] S. Alioli, P. Nason, C. Oleari and E. Re, A general framework for implementing NLO calculations in shower Monte Carlo programs: the POWHEG BOX, JHEP 06 (2010) 043 [arXiv:1002.2581] [INSPIRE].

[58] E. Re, Single-top Wt-channel production matched with parton showers using the POWHEG method, Eur. Phys. J. C 71 (2011) 1547 [arXiv:1009.2450] [INSPIRE].

[59] P. Nason and G. Zanderighi, $W^{+} W^{-}, W Z$ and $Z Z$ production in the POWHEG-BOX-V2, Eur. Phys. J. C 74 (2014) 2702 [arXiv:1311.1365] [INSPIRE].

[60] T. Sjöstrand, S. Mrenna and P.Z. Skands, A brief introduction to PYTHIA 8.1, Comput. Phys. Commun. 178 (2008) 852 [arXiv:0710.3820] [INSPIRE].

[61] J. Alwall et al., Comparative study of various algorithms for the merging of parton showers and matrix elements in hadronic collisions, Eur. Phys. J. C 53 (2008) 473

[arXiv:0706 .2569] [INSPIRE].

[62] R. Frederix and S. Frixione, Merging meets matching in MC@NLO, JHEP 12 (2012) 061 [arXiv:1209.6215] [INSPIRE].

[63] J.M. Campbell, R.K. Ellis, P. Nason and E. Re, Top-pair production and decay at NLO matched with parton showers, JHEP 04 (2015) 114 [arXiv:1412.1828] [INSPIRE]. 
[64] M. Bähr et al., HERWIG++ physics and manual, Eur. Phys. J. C 58 (2008) 639 [arXiv:0803.0883] [INSPIRE].

[65] C. Borschensky et al., Squark and gluino production cross sections in pp collisions at $\sqrt{s}=13,14,33$ and 100 TeV, Eur. Phys. J. C 74 (2014) 3174 [arXiv:1407.5066] [INSPIRE].

[66] M. Czakon and A. Mitov, Top ++ : a program for the calculation of the top-pair cross-section at hadron colliders, Comput. Phys. Commun. 185 (2014) 2930 [arXiv:1112.5675] [INSPIRE].

[67] Y. Li and F. Petriello, Combining QCD and electroweak corrections to dilepton production in FEWZ, Phys. Rev. D 86 (2012) 094034 [arXiv: 1208.5967] [inSPIRE].

[68] P. Kant et al., HatHor for single top-quark production: updated predictions and uncertainty estimates for single top-quark production in hadronic collisions, Comput. Phys. Commun. 191 (2015) 74 [arXiv: 1406.4403] [INSPIRE].

[69] N. Kidonakis, NNLL threshold resummation for top-pair and single-top production, Phys. Part. Nucl. 45 (2014) 714 [arXiv:1210.7813] [INSPIRE].

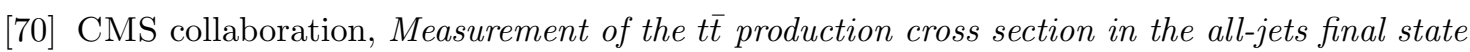
in pp collisions at $\sqrt{s}=8 \mathrm{TeV}$, Eur. Phys. J. C 76 (2016) 128 [arXiv:1509.06076] [INSPIRE].

[71] GEANT4 collaboration, S. Agostinelli et al., GEANT4: a simulation toolkit, Nucl. Instrum. Meth. A 506 (2003) 250 [INSPIRE].

[72] CMS collaboration, The fast simulation of the CMS detector at LHC, J. Phys. Conf. Ser. 331 (2011) 032049 [INSPIRE].

[73] CMS collaboration, Particle-flow reconstruction and global event description with the CMS detector, arXiv:1706.04965 [INSPIRE].

[74] M. Cacciari, G.P. Salam and G. Soyez, The anti-k $k_{t}$ jet clustering algorithm, JHEP 04 (2008) 063 [arXiv: 0802.1189] [INSPIRE].

[75] M. Cacciari, G.P. Salam and G. Soyez, FastJet user manual, Eur. Phys. J. C 72 (2012) 1896 [arXiv:1111.6097] [INSPIRE].

[76] M. Cacciari and G.P. Salam, Pileup subtraction using jet areas, Phys. Lett. B 659 (2008) 119 [arXiv:0707.1378] [INSPIRE].

[77] CMS collaboration, Study of pileup removal algorithms for jets, CMS-PAS-JME-14-001, CERN, Geneva Switzerland, (2014).

[78] CMS collaboration, Jet energy scale and resolution in the CMS experiment in pp collisions at $8 \mathrm{TeV}, 2017$ JINST $12 \mathrm{P} 02014$ [arXiv:1607.03663] [INSPIRE].

[79] CMS collaboration, Identification of b-quark jets with the CMS experiment, 2013 JINST 8 P04013 [arXiv: 1211.4462] [INSPIRE].

[80] CMS collaboration, Identification of b quark jets at the CMS experiment in the LHC run 2, CMS-PAS-BTV-15-001, CERN, Geneva Switzerland, (2015).

[81] CMS collaboration, Performance of electron reconstruction and selection with the CMS detector in proton-proton collisions at $\sqrt{s}=8 \mathrm{TeV}, 2015$ JINST $10 \mathrm{P} 06005$ [arXiv: 1502. 02701] [INSPIRE]. 
[82] CMS collaboration, Performance of CMS muon reconstruction in pp collision events at $\sqrt{s}=7 \mathrm{TeV}, 2012$ JINST 7 P10002 [arXiv:1206.4071] [INSPIRE].

[83] K. Rehermann and B. Tweedie, Efficient identification of boosted semileptonic top quarks at the LHC, JHEP 03 (2011) 059 [arXiv:1007.2221] [INSPIRE].

[84] CMS collaboration, Performance of photon reconstruction and identification with the CMS detector in proton-proton collisions at $\sqrt{s}=8 \mathrm{TeV}, 2015$ JINST $10 \mathrm{P} 08010$ [arXiv: 1502.02702] [INSPIRE].

[85] B.P. Roe, H.-J. Yang, J. Zhu, Y. Liu, I. Stancu and G. McGregor, Boosted decision trees, an alternative to artificial neural networks, Nucl. Instrum. Meth. A 543 (2005) 577 [physics/0408124] [INSPIRE].

[86] J. Shelton, Jet substructure, in Proceedings, Theoretical Advanced Study Institute in Elementary Particle Physics: Searching for New Physics at Small and Large Scales (TASI 2012), Boulder CO U.S.A., June 2013, pg. 303 [arXiv: 1302.0260] [INSPIRE].

[87] Y.L. Dokshitzer, G.D. Leder, S. Moretti and B.R. Webber, Better jet clustering algorithms, JHEP 08 (1997) 001 [hep-ph/9707323] [inSPIRE].

[88] M. Dasgupta, A. Fregoso, S. Marzani and G.P. Salam, Towards an understanding of jet substructure, JHEP 09 (2013) 029 [arXiv:1307.0007] [INSPIRE].

[89] A.J. Larkoski, S. Marzani, G. Soyez and J. Thaler, Soft drop, JHEP 05 (2014) 146 [arXiv: 1402.2657] [INSPIRE].

[90] J. Thaler and K. Van Tilburg, Maximizing boosted top identification by minimizing $N$-subjettiness, JHEP 02 (2012) 093 [arXiv:1108.2701] [INSPIRE].

[91] CMS collaboration, Performance of quark/gluon discrimination in $8 \mathrm{TeV}$ pp data, CMS-PAS-JME-13-002, CERN, Geneva Switzerland, (2013).

[92] A. Höcker et al., TMVA - toolkit for multivariate data analysis, PoS (ACAT) 040 [physics/0703039] [INSPIRE].

[93] CMS collaboration, Jet algorithms performance in $13 \mathrm{TeV}$ data, CMS-PAS-JME-16-003, CERN, Geneva Switzerland, (2016).

[94] CMS collaboration, Identification of c-quark jets at the CMS experiment, CMS-PAS-BTV-16-001, CERN, Geneva Switzerland, (2016).

[95] CMS collaboration, Measurement of $B \bar{B}$ angular correlations based on secondary vertex reconstruction at $\sqrt{s}=7 \mathrm{TeV}$, JHEP 03 (2011) 136 [arXiv:1102.3194] [INSPIRE].

[96] B. Efron, The jackknife, the bootstrap, and other resampling plans, in CBMS-NSF Regional Conference Series in Applied Mathematics 38, SIAM, Philadelphia PA U.S.A., (1982) [ISBN:978-0-898711-79-0].

[97] CMS collaboration, Observation of top quark pairs produced in association with a vector boson in pp collisions at $\sqrt{s}=8 \mathrm{TeV}$, JHEP 01 (2016) 096 [arXiv:1510.01131] [INSPIRE].

[98] ATLAS collaboration, Measurement of the $Z Z$ production cross section in proton-proton collisions at $\sqrt{s}=8 \mathrm{TeV}$ using the $Z Z \rightarrow \ell^{-} \ell^{+} \ell^{\prime-} \ell^{\prime+}$ and $Z Z \rightarrow \ell^{-} \ell^{+} \nu \bar{\nu}$ channels with the ATLAS detector, JHEP 01 (2017) 099 [arXiv: 1610.07585] [INSPIRE].

[99] CMS collaboration, Measurement of the $W Z$ production cross section in pp collisions at $\sqrt{s}=13 \mathrm{TeV}$, Phys. Lett. B 766 (2017) 268 [arXiv:1607.06943] [INSPIRE]. 
[100] S. Catani, D. de Florian, M. Grazzini and P. Nason, Soft gluon resummation for Higgs boson production at hadron colliders, JHEP 07 (2003) 028 [hep-ph/0306211] [INSPIRE].

[101] M. Cacciari, S. Frixione, M.L. Mangano, P. Nason and G. Ridolfi, The t $\bar{t}$ cross-section at $1.8 \mathrm{TeV}$ and $1.96 \mathrm{TeV}$ : a study of the systematics due to parton densities and scale dependence, JHEP 04 (2004) 068 [hep-ph/0303085] [INSPIRE].

[102] CMS collaboration, CMS luminosity measurements for the 2016 data taking period, CMS-PAS-LUM-17-001, CERN, Geneva Switzerland, (2017).

[103] T. Junk, Confidence level computation for combining searches with small statistics, Nucl. Instrum. Meth. A 434 (1999) 435 [hep-ex/9902006] [INSPIRE].

[104] A.L. Read, Presentation of search results: the CLs technique, J. Phys. G 28 (2002) 2693 [INSPIRE].

[105] ATLAS and CMS collaborations, Procedure for the LHC Higgs boson search combination in summer 2011, ATL-PHYS-PUB-2011-011, CERN, Geneva Switzerland, (2011).

[106] G. Cowan, K. Cranmer, E. Gross and O. Vitells, Asymptotic formulae for likelihood-based tests of new physics, Eur. Phys. J. C 71 (2011) 1554 [Erratum ibid. C 73 (2013) 2501] [arXiv: 1007.1727] [INSPIRE].

[107] R. Gröber, M.M. Mühlleitner, E. Popenda and A. Wlotzka, Light stop decays: implications for LHC searches, Eur. Phys. J. C 75 (2015) 420 [arXiv:1408.4662] [inSPIRE]. 


\section{The CMS collaboration}

\section{Yerevan Physics Institute, Yerevan, Armenia}

A.M. Sirunyan, A. Tumasyan

\section{Institut für Hochenergiephysik, Wien, Austria}

W. Adam, F. Ambrogi, E. Asilar, T. Bergauer, J. Brandstetter, E. Brondolin, M. Dragicevic, J. Erö, M. Flechl, M. Friedl, R. Frühwirth ${ }^{1}$, V.M. Ghete, J. Grossmann, J. Hrubec, M. Jeitler ${ }^{1}$, A. König, N. Krammer, I. Krätschmer, D. Liko, T. Madlener, I. Mikulec, E. Pree, D. Rabady, N. Rad, H. Rohringer, J. Schieck ${ }^{1}$, R. Schöfbeck, M. Spanring, D. Spitzbart, J. Strauss, W. Waltenberger, J. Wittmann, C.-E. Wulz ${ }^{1}$, M. Zarucki

Institute for Nuclear Problems, Minsk, Belarus

V. Chekhovsky, V. Mossolov, J. Suarez Gonzalez

\section{Universiteit Antwerpen, Antwerpen, Belgium}

E.A. De Wolf, D. Di Croce, X. Janssen, J. Lauwers, H. Van Haevermaet, P. Van Mechelen, N. Van Remortel

\section{Vrije Universiteit Brussel, Brussel, Belgium}

S. Abu Zeid, F. Blekman, J. D’Hondt, I. De Bruyn, J. De Clercq, K. Deroover, G. Flouris, D. Lontkovskyi, S. Lowette, S. Moortgat, L. Moreels, A. Olbrechts, Q. Python, K. Skovpen, S. Tavernier, W. Van Doninck, P. Van Mulders, I. Van Parijs

\section{Université Libre de Bruxelles, Bruxelles, Belgium}

H. Brun, B. Clerbaux, G. De Lentdecker, H. Delannoy, G. Fasanella, L. Favart, R. Goldouzian, A. Grebenyuk, G. Karapostoli, T. Lenzi, J. Luetic, T. Maerschalk, A. Marinov, A. Randle-conde, T. Seva, C. Vander Velde, P. Vanlaer, D. Vannerom, R. Yonamine, F. Zenoni, F. Zhang ${ }^{2}$

\section{Ghent University, Ghent, Belgium}

A. Cimmino, T. Cornelis, D. Dobur, A. Fagot, M. Gul, I. Khvastunov, D. Poyraz, C. Roskas, S. Salva, M. Tytgat, W. Verbeke, N. Zaganidis

\section{Université Catholique de Louvain, Louvain-la-Neuve, Belgium}

H. Bakhshiansohi, O. Bondu, S. Brochet, G. Bruno, A. Caudron, S. De Visscher, C. Delaere, M. Delcourt, B. Francois, A. Giammanco, A. Jafari, M. Komm, G. Krintiras, V. Lemaitre, A. Magitteri, A. Mertens, M. Musich, K. Piotrzkowski, L. Quertenmont, M. Vidal Marono, S. Wertz

\section{Université de Mons, Mons, Belgium}

N. Beliy

\section{Centro Brasileiro de Pesquisas Fisicas, Rio de Janeiro, Brazil}

W.L. Aldá Júnior, F.L. Alves, G.A. Alves, L. Brito, M. Correa Martins Junior, C. Hensel, A. Moraes, M.E. Pol, P. Rebello Teles 
Universidade do Estado do Rio de Janeiro, Rio de Janeiro, Brazil

E. Belchior Batista Das Chagas, W. Carvalho, J. Chinellato ${ }^{3}$, A. Custódio, E.M. Da Costa, G.G. Da Silveira ${ }^{4}$, D. De Jesus Damiao, S. Fonseca De Souza, L.M. Huertas Guativa, H. Malbouisson, M. Melo De Almeida, C. Mora Herrera, L. Mundim, H. Nogima, A. Santoro, A. Sznajder, E.J. Tonelli Manganote ${ }^{3}$, F. Torres Da Silva De Araujo, A. Vilela Pereira

Universidade Estadual Paulista $^{a}$, Universidade Federal do ABC ${ }^{b}$, São Paulo, Brazil

S. Ahuja ${ }^{a}$, C.A. Bernardes ${ }^{a}$, T.R. Fernandez Perez Tomei ${ }^{a}$, E.M. Gregores ${ }^{b}$, P.G. Mercadante ${ }^{b}$, S.F. Novaes ${ }^{a}$, Sandra S. Padula ${ }^{a}$, D. Romero $\operatorname{Abad}^{b}$, J.C. Ruiz $\operatorname{Vargas}^{a}$

Institute for Nuclear Research and Nuclear Energy of Bulgaria Academy of Sciences

A. Aleksandrov, R. Hadjiiska, P. Iaydjiev, M. Misheva, M. Rodozov, M. Shopova, S. Stoykova, G. Sultanov

University of Sofia, Sofia, Bulgaria

A. Dimitrov, I. Glushkov, L. Litov, B. Pavlov, P. Petkov

Beihang University, Beijing, China

W. Fang ${ }^{5}$, X. Gao $^{5}$

Institute of High Energy Physics, Beijing, China

M. Ahmad, J.G. Bian, G.M. Chen, H.S. Chen, M. Chen, Y. Chen, C.H. Jiang, D. Leggat, H. Liao, Z. Liu, F. Romeo, S.M. Shaheen, A. Spiezia, J. Tao, C. Wang, Z. Wang, E. Yazgan, H. Zhang, J. Zhao

State Key Laboratory of Nuclear Physics and Technology, Peking University, Beijing, China

Y. Ban, G. Chen, Q. Li, S. Liu, Y. Mao, S.J. Qian, D. Wang, Z. Xu

Universidad de Los Andes, Bogota, Colombia

C. Avila, A. Cabrera, L.F. Chaparro Sierra, C. Florez, C.F. González Hernández, J.D. Ruiz Alvarez

University of Split, Faculty of Electrical Engineering, Mechanical Engineering and Naval Architecture, Split, Croatia

B. Courbon, N. Godinovic, D. Lelas, I. Puljak, P.M. Ribeiro Cipriano, T. Sculac

University of Split, Faculty of Science, Split, Croatia

Z. Antunovic, M. Kovac

Institute Rudjer Boskovic, Zagreb, Croatia

V. Brigljevic, D. Ferencek, K. Kadija, B. Mesic, A. Starodumov ${ }^{6}$, T. Susa

University of Cyprus, Nicosia, Cyprus

M.W. Ather, A. Attikis, G. Mavromanolakis, J. Mousa, C. Nicolaou, F. Ptochos, P.A. Razis, H. Rykaczewski 
Charles University, Prague, Czech Republic

M. Finger ${ }^{7}$, M. Finger Jr. ${ }^{7}$

Universidad San Francisco de Quito, Quito, Ecuador

E. Carrera Jarrin

Academy of Scientific Research and Technology of the Arab Republic of Egypt, Egyptian Network of High Energy Physics, Cairo, Egypt

E. El-khateeb ${ }^{8}$, S. Elgammal ${ }^{9}$, A. Mohamed $^{10}$

National Institute of Chemical Physics and Biophysics, Tallinn, Estonia

R.K. Dewanjee, M. Kadastik, L. Perrini, M. Raidal, A. Tiko, C. Veelken

Department of Physics, University of Helsinki, Helsinki, Finland

P. Eerola, J. Pekkanen, M. Voutilainen

Helsinki Institute of Physics, Helsinki, Finland

J. Härkönen, T. Järvinen, V. Karimäki, R. Kinnunen, T. Lampén, K. Lassila-Perini,

S. Lehti, T. Lindén, P. Luukka, E. Tuominen, J. Tuominiemi, E. Tuovinen

Lappeenranta University of Technology, Lappeenranta, Finland

J. Talvitie, T. Tuuva

IRFU, CEA, Université Paris-Saclay, Gif-sur-Yvette, France

M. Besancon, F. Couderc, M. Dejardin, D. Denegri, J.L. Faure, F. Ferri, S. Ganjour, S. Ghosh, A. Givernaud, P. Gras, G. Hamel de Monchenault, P. Jarry, I. Kucher, E. Locci, M. Machet, J. Malcles, G. Negro, J. Rander, A. Rosowsky, M.Ö. Sahin, M. Titov

Laboratoire Leprince-Ringuet, Ecole polytechnique, CNRS/IN2P3, Université Paris-Saclay, Palaiseau, France

A. Abdulsalam, I. Antropov, S. Baffioni, F. Beaudette, P. Busson, L. Cadamuro, C. Charlot, R. Granier de Cassagnac, M. Jo, S. Lisniak, A. Lobanov, J. Martin Blanco, M. Nguyen, C. Ochando, G. Ortona, P. Paganini, P. Pigard, S. Regnard, R. Salerno, J.B. Sauvan, Y. Sirois, A.G. Stahl Leiton, T. Strebler, Y. Yilmaz, A. Zabi

Université de Strasbourg, CNRS, IPHC UMR 7178, F-67000 Strasbourg, France

J.-L. Agram ${ }^{11}$, J. Andrea, D. Bloch, J.-M. Brom, M. Buttignol, E.C. Chabert, N. Chanon, C. Collard, E. Conte ${ }^{11}$, X. Coubez, J.-C. Fontaine ${ }^{11}$, D. Gelé, U. Goerlach, M. Jansová, A.-C. Le Bihan, N. Tonon, P. Van Hove

Centre de Calcul de l'Institut National de Physique Nucleaire et de Physique des Particules, CNRS/IN2P3, Villeurbanne, France

S. Gadrat

Université de Lyon, Université Claude Bernard Lyon 1, CNRS-IN2P3, Institut de Physique Nucléaire de Lyon, Villeurbanne, France

S. Beauceron, C. Bernet, G. Boudoul, R. Chierici, D. Contardo, P. Depasse, H. El Mamouni, J. Fay, L. Finco, S. Gascon, M. Gouzevitch, G. Grenier, B. Ille, F. Lagarde, I.B. Laktineh, 
M. Lethuillier, L. Mirabito, A.L. Pequegnot, S. Perries, A. Popov ${ }^{12}$, V. Sordini, M. Vander Donckt, S. Viret

\section{Georgian Technical University, Tbilisi, Georgia}

A. Khvedelidze ${ }^{7}$

Tbilisi State University, Tbilisi, Georgia

Z. Tsamalaidze ${ }^{7}$

RWTH Aachen University, I. Physikalisches Institut, Aachen, Germany

C. Autermann, S. Beranek, L. Feld, M.K. Kiesel, K. Klein, M. Lipinski, M. Preuten, C. Schomakers, J. Schulz, T. Verlage

RWTH Aachen University, III. Physikalisches Institut A, Aachen, Germany A. Albert, E. Dietz-Laursonn, D. Duchardt, M. Endres, M. Erdmann, S. Erdweg, T. Esch, R. Fischer, A. Güth, M. Hamer, T. Hebbeker, C. Heidemann, K. Hoepfner, S. Knutzen, M. Merschmeyer, A. Meyer, P. Millet, S. Mukherjee, M. Olschewski, K. Padeken, T. Pook, M. Radziej, H. Reithler, M. Rieger, F. Scheuch, D. Teyssier, S. Thüer

RWTH Aachen University, III. Physikalisches Institut B, Aachen, Germany

G. Flügge, B. Kargoll, T. Kress, A. Künsken, J. Lingemann, T. Müller, A. Nehrkorn, A. Nowack, C. Pistone, O. Pooth, A. Stahl ${ }^{13}$

\section{Deutsches Elektronen-Synchrotron, Hamburg, Germany}

M. Aldaya Martin, T. Arndt, C. Asawatangtrakuldee, K. Beernaert, O. Behnke, U. Behrens, A. Bermúdez Martínez, A.A. Bin Anuar, K. Borras ${ }^{14}$, V. Botta, A. Campbell, P. Connor, C. Contreras-Campana, F. Costanza, C. Diez Pardos, G. Eckerlin, D. Eckstein, T. Eichhorn, E. Eren, E. Gallo ${ }^{15}$, J. Garay Garcia, A. Geiser, A. Gizhko, J.M. Grados Luyando, A. Grohsjean, P. Gunnellini, A. Harb, J. Hauk, M. Hempel ${ }^{16}$, H. Jung, A. Kalogeropoulos, M. Kasemann, J. Keaveney, C. Kleinwort, I. Korol, D. Krücker, W. Lange, A. Lelek, T. Lenz, J. Leonard, K. Lipka, W. Lohmann ${ }^{16}$, R. Mankel, I.A. Melzer-Pellmann, A.B. Meyer, G. Mittag, J. Mnich, A. Mussgiller, E. Ntomari, D. Pitzl, R. Placakyte, A. Raspereza, B. Roland, M. Savitskyi, P. Saxena, R. Shevchenko, S. Spannagel, N. Stefaniuk, G.P. Van Onsem, R. Walsh, Y. Wen, K. Wichmann, C. Wissing, O. Zenaiev

\section{University of Hamburg, Hamburg, Germany}

S. Bein, V. Blobel, M. Centis Vignali, A.R. Draeger, T. Dreyer, E. Garutti, D. Gonzalez, J. Haller, A. Hinzmann, M. Hoffmann, A. Karavdina, R. Klanner, R. Kogler, N. Kovalchuk, S. Kurz, T. Lapsien, I. Marchesini, D. Marconi, M. Meyer, M. Niedziela, D. Nowatschin, F. Pantaleo ${ }^{13}$, T. Peiffer, A. Perieanu, C. Scharf, P. Schleper, A. Schmidt, S. Schumann, J. Schwandt, J. Sonneveld, H. Stadie, G. Steinbrück, F.M. Stober, M. Stöver, H. Tholen, D. Troendle, E. Usai, L. Vanelderen, A. Vanhoefer, B. Vormwald

Institut für Experimentelle Kernphysik, Karlsruhe, Germany

M. Akbiyik, C. Barth, S. Baur, E. Butz, R. Caspart, T. Chwalek, F. Colombo, W. De Boer, A. Dierlamm, B. Freund, R. Friese, M. Giffels, A. Gilbert, D. Haitz, F. Hartmann ${ }^{13}$, 
S.M. Heindl, U. Husemann, F. Kassel ${ }^{13}$, S. Kudella, H. Mildner, M.U. Mozer, Th. Müller, M. Plagge, G. Quast, K. Rabbertz, M. Schröder, I. Shvetsov, G. Sieber, H.J. Simonis,

R. Ulrich, S. Wayand, M. Weber, T. Weiler, S. Williamson, C. Wöhrmann, R. Wolf

Institute of Nuclear and Particle Physics (INPP), NCSR Demokritos, Aghia Paraskevi, Greece

G. Anagnostou, G. Daskalakis, T. Geralis, V.A. Giakoumopoulou, A. Kyriakis, D. Loukas, I. Topsis-Giotis

National and Kapodistrian University of Athens, Athens, Greece

S. Kesisoglou, A. Panagiotou, N. Saoulidou

University of Ioánnina, Ioánnina, Greece

I. Evangelou, C. Foudas, P. Kokkas, S. Mallios, N. Manthos, I. Papadopoulos, E. Paradas, J. Strologas, F.A. Triantis

MTA-ELTE Lendület CMS Particle and Nuclear Physics Group, Eötvös Loránd University, Budapest, Hungary

M. Csanad, N. Filipovic, G. Pasztor

Wigner Research Centre for Physics, Budapest, Hungary

G. Bencze, C. Hajdu, D. Horvath ${ }^{17}$, Á. Hunyadi, F. Sikler, V. Veszpremi, G. Vesztergombi ${ }^{18}$, A.J. Zsigmond

Institute of Nuclear Research ATOMKI, Debrecen, Hungary

N. Beni, S. Czellar, J. Karancsi ${ }^{19}$, A. Makovec, J. Molnar, Z. Szillasi

Institute of Physics, University of Debrecen, Debrecen, Hungary

M. Bartók ${ }^{18}$, P. Raics, Z.L. Trocsanyi, B. Ujvari

Indian Institute of Science (IISc), Bangalore, India

S. Choudhury, J.R. Komaragiri

National Institute of Science Education and Research, Bhubaneswar, India

S. Bahinipati ${ }^{20}$, S. Bhowmik, P. Mal, K. Mandal, A. Nayak ${ }^{21}$, D.K. Sahoo ${ }^{20}$, N. Sahoo, S.K. Swain

Panjab University, Chandigarh, India

S. Bansal, S.B. Beri, V. Bhatnagar, U. Bhawandeep, R. Chawla, N. Dhingra, A.K. Kalsi, A. Kaur, M. Kaur, R. Kumar, P. Kumari, A. Mehta, J.B. Singh, G. Walia

University of Delhi, Delhi, India

Ashok Kumar, Aashaq Shah, A. Bhardwaj, S. Chauhan, B.C. Choudhary, R.B. Garg, S. Keshri, A. Kumar, S. Malhotra, M. Naimuddin, K. Ranjan, R. Sharma, V. Sharma

Saha Institute of Nuclear Physics, HBNI, Kolkata, India

R. Bhardwaj, R. Bhattacharya, S. Bhattacharya, S. Dey, S. Dutt, S. Dutta, S. Ghosh, N. Majumdar, A. Modak, K. Mondal, S. Mukhopadhyay, S. Nandan, A. Purohit, A. Roy, D. Roy, S. Roy Chowdhury, S. Sarkar, M. Sharan, S. Thakur 
Indian Institute of Technology Madras, Madras, India

P.K. Behera

Bhabha Atomic Research Centre, Mumbai, India

R. Chudasama, D. Dutta, V. Jha, V. Kumar, A.K. Mohanty ${ }^{13}$, P.K. Netrakanti, L.M. Pant, P. Shukla, A. Topkar

Tata Institute of Fundamental Research-A, Mumbai, India

T. Aziz, S. Dugad, B. Mahakud, S. Mitra, G.B. Mohanty, B. Parida, N. Sur, B. Sutar

Tata Institute of Fundamental Research-B, Mumbai, India

S. Banerjee, S. Bhattacharya, S. Chatterjee, P. Das, M. Guchait, Sa. Jain, S. Kumar, M. Maity ${ }^{22}$, G. Majumder, K. Mazumdar, T. Sarkar ${ }^{22}$, N. Wickramage ${ }^{23}$

Indian Institute of Science Education and Research (IISER), Pune, India

S. Chauhan, S. Dube, V. Hegde, A. Kapoor, K. Kothekar, S. Pandey, A. Rane, S. Sharma

Institute for Research in Fundamental Sciences (IPM), Tehran, Iran

S. Chenarani ${ }^{24}$, E. Eskandari Tadavani, S.M. Etesami ${ }^{24}$, M. Khakzad, M. Mohammadi Najafabadi, M. Naseri, S. Paktinat Mehdiabadi ${ }^{25}$, F. Rezaei Hosseinabadi, B. Safarzadeh ${ }^{26}$, M. Zeinali

University College Dublin, Dublin, Ireland

M. Felcini, M. Grunewald

INFN Sezione di Bari ${ }^{a}$, Università di Bari ${ }^{b}$, Politecnico di Bari ${ }^{c}$, Bari, Italy M. Abbrescia ${ }^{a, b}$, C. Calabria ${ }^{a, b}$, C. Caputo ${ }^{a, b}$, A. Colaleo $^{a}$, D. Creanza ${ }^{a, c}$, L. Cristella $^{a, b}$, N. De Filippis ${ }^{a, c}$, M. De Palma ${ }^{a, b}$, F. Errico ${ }^{a, b}$, L. Fiore $^{a}$, G. Iasellia ${ }^{a, c}$, S. Lezki $^{a, b}$,

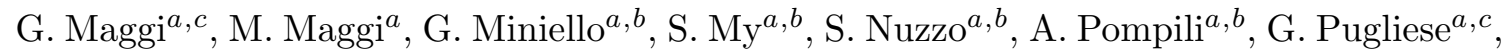
R. Radogna ${ }^{a, b}$, A. Ranieri ${ }^{a}$, G. Selvaggi ${ }^{a}, b$ A. Sharma ${ }^{a}$, L. Silvestris ${ }^{a, 13}$, R. Venditti ${ }^{a}$, P. Verwilligen ${ }^{a}$

INFN Sezione di Bologna ${ }^{a}$, Università di Bologna ${ }^{b}$, Bologna, Italy

G. Abbiendi ${ }^{a}$, C. Battilana ${ }^{a, b}$, D. Bonacorsi ${ }^{a}, b$, S. Braibant-Giacomelli ${ }^{a}, b$, R. Campanini $^{a, b}{ }$, P. Capiluppi ${ }^{a, b}$, A. Castro ${ }^{a, b}$, F.R. Cavallo ${ }^{a}$, S.S. Chhibra ${ }^{a}$, G. Codispoti ${ }^{a, b}$, M. Cuffiani ${ }^{a, b}$, G.M. Dallavalle ${ }^{a}$, F. Fabbri ${ }^{a}$, A. Fanfani ${ }^{a}, b$, D. Fasanella ${ }^{a, b}$, P. Giacomelli ${ }^{a}$, C. Grandi ${ }^{a}$, L. Guiducci ${ }^{a}, b$, S. Marcellini ${ }^{a}$, G. Masetti ${ }^{a}$, A. Montanari ${ }^{a}$, F.L. Navarria ${ }^{a, b}$, A. Perrotta ${ }^{a}$, A.M. Rossi ${ }^{a, b}$, T. Rovelli ${ }^{a, b}$, G.P. Siroli ${ }^{a, b}$, N. Tosi ${ }^{a}$

INFN Sezione di Catania ${ }^{a}$, Università di Catania ${ }^{b}$, Catania, Italy

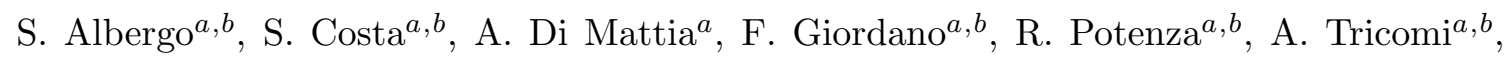
C. Tuve ${ }^{a, b}$

INFN Sezione di Firenze ${ }^{a}$, Università di Firenze ${ }^{b}$, Firenze, Italy

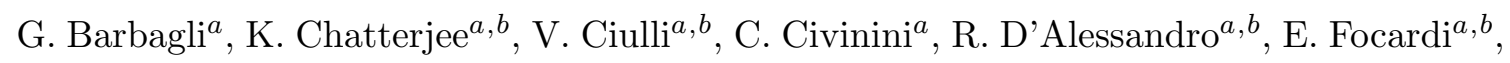

P. Lenzi ${ }^{a, b}$, M. Meschini ${ }^{a}$, S. Paoletti ${ }^{a}$, L. Russo ${ }^{a, 27}$, G. Sguazzoni ${ }^{a}$, D. Strom $^{a}$,

L. Viliani ${ }^{a, b, 13}$ 
INFN Laboratori Nazionali di Frascati, Frascati, Italy

L. Benussi, S. Bianco, F. Fabbri, D. Piccolo, F. Primavera ${ }^{13}$

INFN Sezione di Genova ${ }^{a}$, Università di Genova ${ }^{b}$, Genova, Italy

V. Calvelli ${ }^{a, b}$, F. Ferro ${ }^{a}$, E. Robutti $^{a}$, S. Tosi $^{a, b}$

INFN Sezione di Milano-Bicocca ${ }^{a}$, Università di Milano-Bicocca ${ }^{b}$, Milano, Italy

L. Brianza ${ }^{a, b}$, F. Brivio ${ }^{a, b}$, V. Ciriolo ${ }^{a, b}$, M.E. Dinardo ${ }^{a, b}$, S. Fiorendi ${ }^{a, b}$, S. Gennai $^{a}$,

A. Ghezzi ${ }^{a, b}$, P. Govoni ${ }^{a, b}$, M. Malberti ${ }^{a, b}$, S. Malvezzi ${ }^{a}$, R.A. Manzoni ${ }^{a, b}$, D. Menasce ${ }^{a}$,

L. Moroni ${ }^{a}$, M. Paganoni ${ }^{a, b}$, K. Pauwels ${ }^{a, b}$, D. Pedrini ${ }^{a}$, S. Pigazzini ${ }^{a, b, 28}$, S. Ragazzi ${ }^{a, b}$,

T. Tabarelli de Fatis ${ }^{a, b}$

INFN Sezione di Napoli ${ }^{a}$, Università di Napoli 'Federico II' ${ }^{b}$, Napoli, Italy, Università della Basilicata ${ }^{c}$, Potenza, Italy, Università G. Marconi ${ }^{d}$, Roma, Italy

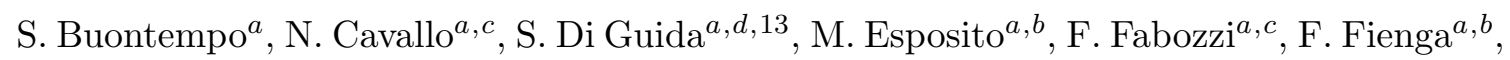
A.O.M. Iorio ${ }^{a, b}$, W.A. Khan ${ }^{a}$, G. Lanza ${ }^{a}$, L. Lista ${ }^{a}$, S. Meola ${ }^{a, d, 13}$, P. Paolucci ${ }^{a, 13}$, C. Sciacca ${ }^{a, b}$, F. Thyssen ${ }^{a}$

INFN Sezione di Padova ${ }^{a}$, Università di Padova ${ }^{b}$, Padova, Italy, Università di Trento ${ }^{c}$, Trento, Italy

P. Azzi ${ }^{a, 13}$, N. Bacchetta ${ }^{a}$, L. Benato ${ }^{a, b}$, D. Bisello ${ }^{a, b}$, A. Boletti ${ }^{a, b}$, R. Carlin ${ }^{a, b}$, A. Carvalho Antunes De Oliveira ${ }^{a, b}$, P. Checchia ${ }^{a}$, M. Dall'Osso ${ }^{a, b}$, P. De Castro Manzano ${ }^{a}$,

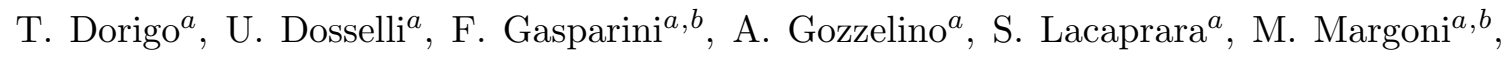
G. Maron ${ }^{a, 29}$, A.T. Meneguzzo ${ }^{a, b}$, M. Michelotto ${ }^{a}$, F. Montecassiano ${ }^{a}$, N. Pozzobon ${ }^{a, b}$, P. Ronchese ${ }^{a, b}$, R. Rossin ${ }^{a, b}$, E. Torassa ${ }^{a}$, M. Zanetti ${ }^{a, b}$, P. Zotto ${ }^{a, b}$

INFN Sezione di Pavia ${ }^{a}$, Università di Pavia ${ }^{b}$, Pavia, Italy

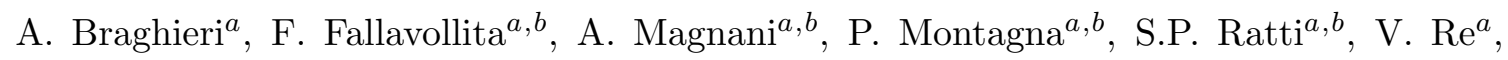
M. Ressegotti, C. Riccardi ${ }^{a, b}$, P. Salvini ${ }^{a}$, I. Vai ${ }^{a, b}$, P. Vitulo ${ }^{a, b}$

INFN Sezione di Perugia ${ }^{a}$, Università di Perugia ${ }^{b}$, Perugia, Italy

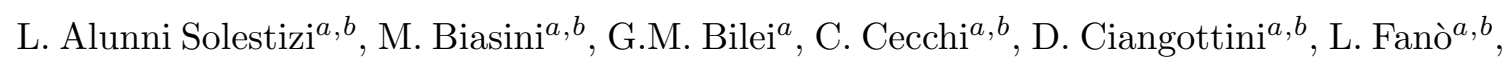
P. Lariccia ${ }^{a, b}$, R. Leonardi ${ }^{a, b}$, E. Manoni $^{a}$, G. Mantovani ${ }^{a, b}$, V. Mariani $^{a, b}$, M. Menichelli $^{a}$, A. $\operatorname{Rossi}^{a, b}$, A. Santocchia ${ }^{a, b}$, D. Spiga ${ }^{a}$

INFN Sezione di Pisa ${ }^{a}$, Università di Pisa ${ }^{b}$, Scuola Normale Superiore di Pisa ${ }^{c}$, Pisa, Italy

K. Androsov ${ }^{a}$, P. Azzurri ${ }^{a, 13}$, G. Bagliesi ${ }^{a}$, J. Bernardini ${ }^{a}$, T. Boccali ${ }^{a}$, L. Borrello,

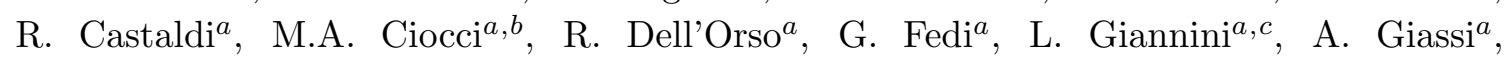
M.T. Grippo ${ }^{a, 27}$, F. Ligabue ${ }^{a, c}$, T. Lomtadze ${ }^{a}$, E. Manca ${ }^{a, c}$, G. Mandorli ${ }^{a, c}$, L. Martini $^{a, b}$, A. Messineo ${ }^{a, b}$, F. Palla ${ }^{a}$, A. Rizzi ${ }^{a, b}$, A. Savoy-Navarro ${ }^{a, 30}$, P. Spagnolo $^{a}$, R. Tenchini ${ }^{a}$, G. Tonelli ${ }^{a, b}$, A. Venturi ${ }^{a}$, P.G. Verdini ${ }^{a}$ 
INFN Sezione di Roma ${ }^{a}$, Sapienza Università di Roma ${ }^{b}$, Rome, Italy

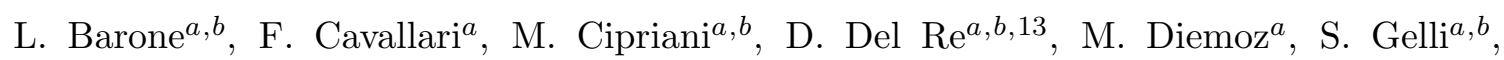
E. Longo $^{a, b}$, F. Margaroli ${ }^{a, b}$, B. Marzocchi ${ }^{a, b}$, P. Meridiani ${ }^{a}$, G. Organtini ${ }^{a, b}$,

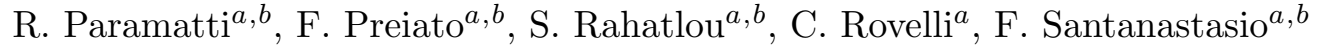

INFN Sezione di Torino ${ }^{a}$, Università di Torino ${ }^{b}$, Torino, Italy, Università del Piemonte Orientale ${ }^{c}$, Novara, Italy

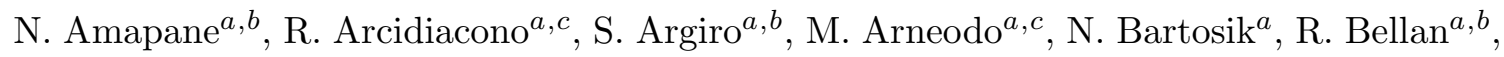

C. Biino ${ }^{a}$, N. Cartiglia ${ }^{a}$, F. Cenna ${ }^{a, b}$, M. Costa ${ }^{a, b}$, R. Covarelli ${ }^{a, b}$, A. Degano ${ }^{a, b}$,

N. Demaria ${ }^{a}$, B. Kiani ${ }^{a}, b$ C. Mariotti ${ }^{a}$, S. Maselli ${ }^{a}$, E. Migliore ${ }^{a, b}$, V. Monaco ${ }^{a, b}$,

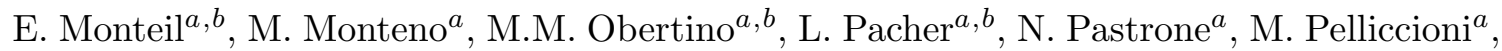
G.L. Pinna Angioni ${ }^{a, b}$, F. Ravera ${ }^{a, b}$, A. Romero ${ }^{a, b}$, M. Ruspa ${ }^{a, c}$, R. Sacchi ${ }^{a, b}$,

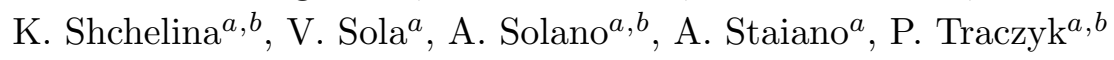

INFN Sezione di Trieste ${ }^{a}$, Università di Trieste ${ }^{b}$, Trieste, Italy

S. Belforte ${ }^{a}$, M. Casarsa ${ }^{a}$, F. Cossutti ${ }^{a}$, G. Della Ricca ${ }^{a, b}$, A. Zanetti $^{a}$

Kyungpook National University, Daegu, Korea

D.H. Kim, G.N. Kim, M.S. Kim, J. Lee, S. Lee, S.W. Lee, C.S. Moon, Y.D. Oh, S. Sekmen, D.C. Son, Y.C. Yang

Chonbuk National University, Jeonju, Korea

A. Lee

Chonnam National University, Institute for Universe and Elementary Particles, Kwangju, Korea

H. Kim, D.H. Moon, G. Oh

Hanyang University, Seoul, Korea

J.A. Brochero Cifuentes, J. Goh, T.J. Kim

Korea University, Seoul, Korea

S. Cho, S. Choi, Y. Go, D. Gyun, S. Ha, B. Hong, Y. Jo, Y. Kim, K. Lee, K.S. Lee, S. Lee, J. Lim, S.K. Park, Y. Roh

Seoul National University, Seoul, Korea

J. Almond, J. Kim, J.S. Kim, H. Lee, K. Lee, K. Nam, S.B. Oh, B.C. Radburn-Smith, S.h. Seo, U.K. Yang, H.D. Yoo, G.B. Yu

University of Seoul, Seoul, Korea

M. Choi, H. Kim, J.H. Kim, J.S.H. Lee, I.C. Park, G. Ryu

Sungkyunkwan University, Suwon, Korea

Y. Choi, C. Hwang, J. Lee, I. Yu

Vilnius University, Vilnius, Lithuania

V. Dudenas, A. Juodagalvis, J. Vaitkus 
National Centre for Particle Physics, Universiti Malaya, Kuala Lumpur, Malaysia

I. Ahmed, Z.A. Ibrahim, M.A.B. Md Ali ${ }^{31}$, F. Mohamad Idris ${ }^{32}$, W.A.T. Wan Abdullah, M.N. Yusli, Z. Zolkapli

Centro de Investigacion y de Estudios Avanzados del IPN, Mexico City, Mexico

H. Castilla-Valdez, E. De La Cruz-Burelo, I. Heredia-De La Cruz ${ }^{33}$, R. Lopez-Fernandez, J. Mejia Guisao, A. Sanchez-Hernandez

Universidad Iberoamericana, Mexico City, Mexico

S. Carrillo Moreno, C. Oropeza Barrera, F. Vazquez Valencia

Benemerita Universidad Autonoma de Puebla, Puebla, Mexico

I. Pedraza, H.A. Salazar Ibarguen, C. Uribe Estrada

Universidad Autónoma de San Luis Potosí, San Luis Potosí, Mexico

A. Morelos Pineda

University of Auckland, Auckland, New Zealand

D. Krofcheck

University of Canterbury, Christchurch, New Zealand

P.H. Butler

National Centre for Physics, Quaid-I-Azam University, Islamabad, Pakistan

A. Ahmad, M. Ahmad, Q. Hassan, H.R. Hoorani, A. Saddique, M.A. Shah, M. Shoaib, M. Waqas

National Centre for Nuclear Research, Swierk, Poland

H. Bialkowska, M. Bluj, B. Boimska, T. Frueboes, M. Górski, M. Kazana, K. Nawrocki,

K. Romanowska-Rybinska, M. Szleper, P. Zalewski

Institute of Experimental Physics, Faculty of Physics, University of Warsaw, Warsaw, Poland

K. Bunkowski, A. Byszuk ${ }^{34}$, K. Doroba, A. Kalinowski, M. Konecki, J. Krolikowski, M. Misiura, M. Olszewski, A. Pyskir, M. Walczak

Laboratório de Instrumentação e Física Experimental de Partículas, Lisboa, Portugal

P. Bargassa, C. Beirão Da Cruz E Silva, B. Calpas, A. Di Francesco, P. Faccioli, M. Gallinaro, J. Hollar, N. Leonardo, L. Lloret Iglesias, M.V. Nemallapudi, J. Seixas, O. Toldaiev, D. Vadruccio, J. Varela

Joint Institute for Nuclear Research, Dubna, Russia

S. Afanasiev, P. Bunin, M. Gavrilenko, I. Golutvin, I. Gorbunov, A. Kamenev, V. Karjavin, A. Lanev, A. Malakhov, V. Matveev ${ }^{35,36}$, V. Palichik, V. Perelygin, S. Shmatov, S. Shulha, N. Skatchkov, V. Smirnov, N. Voytishin, A. Zarubin 
Petersburg Nuclear Physics Institute, Gatchina (St. Petersburg), Russia

Y. Ivanov, V. Kim ${ }^{37}$, E. Kuznetsova ${ }^{38}$, P. Levchenko, V. Murzin, V. Oreshkin, I. Smirnov, V. Sulimov, L. Uvarov, S. Vavilov, A. Vorobyev

Institute for Nuclear Research, Moscow, Russia

Yu. Andreev, A. Dermenev, S. Gninenko, N. Golubev, A. Karneyeu, M. Kirsanov, N. Krasnikov, A. Pashenkov, D. Tlisov, A. Toropin

Institute for Theoretical and Experimental Physics, Moscow, Russia

V. Epshteyn, V. Gavrilov, N. Lychkovskaya, V. Popov, I. Pozdnyakov, G. Safronov, A. Spiridonov, A. Stepennov, M. Toms, E. Vlasov, A. Zhokin

Moscow Institute of Physics and Technology, Moscow, Russia

T. Aushev, A. Bylinkin ${ }^{36}$

National Research Nuclear University 'Moscow Engineering Physics Institute' (MEPhI), Moscow, Russia

M. Chadeeva ${ }^{39}$, P. Parygin, D. Philippov, S. Polikarpov, E. Popova, V. Rusinov

P.N. Lebedev Physical Institute, Moscow, Russia

V. Andreev, M. Azarkin ${ }^{36}$, I. Dremin ${ }^{36}$, M. Kirakosyan ${ }^{36}$, A. Terkulov

Skobeltsyn Institute of Nuclear Physics, Lomonosov Moscow State University, Moscow, Russia

A. Baskakov, A. Belyaev, E. Boos, V. Bunichev, M. Dubinin ${ }^{40}$, L. Dudko, A. Ershov, V. Klyukhin, O. Kodolova, I. Lokhtin, I. Miagkov, S. Obraztsov, S. Petrushanko, V. Savrin, A. Snigirev

Novosibirsk State University (NSU), Novosibirsk, Russia

V. Blinov ${ }^{41}$, Y.Skovpen ${ }^{41}$, D. Shtol ${ }^{41}$

State Research Center of Russian Federation, Institute for High Energy Physics, Protvino, Russia

I. Azhgirey, I. Bayshev, S. Bitioukov, D. Elumakhov, V. Kachanov, A. Kalinin, D. Konstantinov, V. Krychkine, V. Petrov, R. Ryutin, A. Sobol, S. Troshin, N. Tyurin, A. Uzunian, A. Volkov

University of Belgrade, Faculty of Physics and Vinca Institute of Nuclear Sciences, Belgrade, Serbia

P. Adzic ${ }^{42}$, P. Cirkovic, D. Devetak, M. Dordevic, J. Milosevic, V. Rekovic

Centro de Investigaciones Energéticas Medioambientales y Tecnológicas (CIEMAT), Madrid, Spain

J. Alcaraz Maestre, M. Barrio Luna, M. Cerrada, N. Colino, B. De La Cruz, A. Delgado Peris, A. Escalante Del Valle, C. Fernandez Bedoya, J.P. Fernández Ramos, J. Flix, M.C. Fouz, P. Garcia-Abia, O. Gonzalez Lopez, S. Goy Lopez, J.M. Hernandez, M.I. Josa, A. Pérez-Calero Yzquierdo, J. Puerta Pelayo, A. Quintario Olmeda, I. Redondo, L. Romero, M.S. Soares, A. Álvarez Fernández 
Universidad Autónoma de Madrid, Madrid, Spain

J.F. de Trocóniz, M. Missiroli, D. Moran

Universidad de Oviedo, Oviedo, Spain

J. Cuevas, C. Erice, J. Fernandez Menendez, I. Gonzalez Caballero, J.R. González Fernández, E. Palencia Cortezon, S. Sanchez Cruz, I. Suárez Andrés, P. Vischia, J.M. Vizan Garcia

Instituto de Física de Cantabria (IFCA), CSIC-Universidad de Cantabria, Santander, Spain

I.J. Cabrillo, A. Calderon, B. Chazin Quero, E. Curras, M. Fernandez, J. Garcia-Ferrero,

G. Gomez, A. Lopez Virto, J. Marco, C. Martinez Rivero, P. Martinez Ruiz del Arbol,

F. Matorras, J. Piedra Gomez, T. Rodrigo, A. Ruiz-Jimeno, L. Scodellaro, N. Trevisani,

I. Vila, R. Vilar Cortabitarte

CERN, European Organization for Nuclear Research, Geneva, Switzerland

D. Abbaneo, E. Auffray, P. Baillon, A.H. Ball, D. Barney, M. Bianco, P. Bloch, A. Bocci, C. Botta, T. Camporesi, R. Castello, M. Cepeda, G. Cerminara, E. Chapon, Y. Chen, D. d'Enterria, A. Dabrowski, V. Daponte, A. David, M. De Gruttola, A. De Roeck, E. Di $\mathrm{Marco}^{43}$, M. Dobson, B. Dorney, T. du Pree, M. Dünser, N. Dupont, A. Elliott-Peisert, P. Everaerts, G. Franzoni, J. Fulcher, W. Funk, D. Gigi, K. Gill, F. Glege, D. Gulhan, S. Gundacker, M. Guthoff, P. Harris, J. Hegeman, V. Innocente, P. Janot, O. Karacheban ${ }^{16}$, J. Kieseler, H. Kirschenmann, V. Knünz, A. Kornmayer ${ }^{13}$, M.J. Kortelainen, C. Lange, P. Lecoq, C. Lourenço, M.T. Lucchini, L. Malgeri, M. Mannelli, A. Martelli, F. Meijers, J.A. Merlin, S. Mersi, E. Meschi, P. Milenovic ${ }^{44}$, F. Moortgat, M. Mulders, H. Neugebauer, S. Orfanelli, L. Orsini, L. Pape, E. Perez, M. Peruzzi, A. Petrilli, G. Petrucciani, A. Pfeiffer, M. Pierini, A. Racz, T. Reis, G. Rolandi ${ }^{45}$, M. Rovere, H. Sakulin, C. Schäfer, C. Schwick, M. Seidel, M. Selvaggi, A. Sharma, P. Silva, P. Sphicas ${ }^{46}$, J. Steggemann, M. Stoye, M. Tosi, D. Treille, A. Triossi, A. Tsirou, V. Veckalns ${ }^{47}$, G.I. Veres ${ }^{18}$, M. Verweij, N. Wardle, W.D. Zeuner

\section{Paul Scherrer Institut, Villigen, Switzerland}

W. Bertl ${ }^{\dagger}$, L. Caminada ${ }^{48}$, K. Deiters, W. Erdmann, R. Horisberger, Q. Ingram, H.C. Kaestli, D. Kotlinski, U. Langenegger, T. Rohe, S.A. Wiederkehr

Institute for Particle Physics, ETH Zurich, Zurich, Switzerland

F. Bachmair, L. Bäni, P. Berger, L. Bianchini, B. Casal, G. Dissertori, M. Dittmar, M. Donegà, C. Grab, C. Heidegger, D. Hits, J. Hoss, G. Kasieczka, T. Klijnsma, W. Lustermann, B. Mangano, M. Marionneau, M.T. Meinhard, D. Meister, F. Micheli, P. Musella, F. Nessi-Tedaldi, F. Pandolfi, J. Pata, F. Pauss, G. Perrin, L. Perrozzi, M. Quittnat, M. Schönenberger, L. Shchutska, V.R. Tavolaro, K. Theofilatos, M.L. Vesterbacka Olsson, R. Wallny, A. Zagozdzinska ${ }^{34}$, D.H. Zhu

Universität Zürich, Zurich, Switzerland

T.K. Aarrestad, C. Amsler ${ }^{49}$, M.F. Canelli, A. De Cosa, S. Donato, C. Galloni, T. Hreus, B. Kilminster, J. Ngadiuba, D. Pinna, G. Rauco, P. Robmann, D. Salerno, C. Seitz, 
A. Zucchetta

National Central University, Chung-Li, Taiwan

V. Candelise, T.H. Doan, Sh. Jain, R. Khurana, C.M. Kuo, W. Lin, A. Pozdnyakov, S.S. Yu

National Taiwan University (NTU), Taipei, Taiwan

Arun Kumar, P. Chang, Y. Chao, K.F. Chen, P.H. Chen, F. Fiori, W.-S. Hou, Y. Hsiung, Y.F. Liu, R.-S. Lu, M. Miñano Moya, E. Paganis, A. Psallidas, J.f. Tsai

Chulalongkorn University, Faculty of Science, Department of Physics, Bangkok, Thailand

B. Asavapibhop, K. Kovitanggoon, G. Singh, N. Srimanobhas

Çukurova University, Physics Department, Science and Art Faculty, Adana, Turkey

A. Adiguzel ${ }^{50}$, M.N. Bakirci ${ }^{51}$, F. Boran, S. Cerci $^{52}$, S. Damarseckin, Z.S. Demiroglu, C. Dozen, I. Dumanoglu, S. Girgis, G. Gokbulut, Y. Guler, I. $\operatorname{Hos}^{53}$, E.E. Kangal ${ }^{54}$,

O. Kara, A. Kayis Topaksu, U. Kiminsu, M. Oglakci, G. Onengut ${ }^{55}$, K. Ozdemir ${ }^{56}$,

B. Tali ${ }^{52}$, S. Turkcapar, I.S. Zorbakir, C. Zorbilmez

Middle East Technical University, Physics Department, Ankara, Turkey

B. Bilin, G. Karapinar ${ }^{57}$, K. Ocalan ${ }^{58}$, M. Yalvac, M. Zeyrek

Bogazici University, Istanbul, Turkey

E. Gülmez, M. Kaya ${ }^{59}$, O. Kaya ${ }^{60}$, S. Tekten, E.A. Yetkin ${ }^{61}$

Istanbul Technical University, Istanbul, Turkey

M.N. Agaras, S. Atay, A. Cakir, K. Cankocak

Institute for Scintillation Materials of National Academy of Science of Ukraine, Kharkov, Ukraine

B. Grynyov

National Scientific Center, Kharkov Institute of Physics and Technology, Kharkov, Ukraine

L. Levchuk, P. Sorokin

University of Bristol, Bristol, United Kingdom

R. Aggleton, F. Ball, L. Beck, J.J. Brooke, D. Burns, E. Clement, D. Cussans, O. Davignon,

H. Flacher, J. Goldstein, M. Grimes, G.P. Heath, H.F. Heath, J. Jacob, L. Kreczko,

C. Lucas, D.M. Newbold ${ }^{62}$, S. Paramesvaran, A. Poll, T. Sakuma, S. Seif El Nasr-storey,

D. Smith, V.J. Smith

Rutherford Appleton Laboratory, Didcot, United Kingdom

K.W. Bell, A. Belyaev ${ }^{63}$, C. Brew, R.M. Brown, L. Calligaris, D. Cieri, D.J.A. Cockerill, J.A. Coughlan, K. Harder, S. Harper, E. Olaiya, D. Petyt, C.H. Shepherd-Themistocleous, A. Thea, I.R. Tomalin, T. Williams 


\section{Imperial College, London, United Kingdom}

R. Bainbridge, S. Breeze, O. Buchmuller, A. Bundock, S. Casasso, M. Citron, D. Colling, L. Corpe, P. Dauncey, G. Davies, A. De Wit, M. Della Negra, R. Di Maria, A. Elwood, Y. Haddad, G. Hall, G. Iles, T. James, R. Lane, C. Laner, L. Lyons, A.-M. Magnan, S. Malik, L. Mastrolorenzo, T. Matsushita, J. Nash, A. Nikitenko ${ }^{6}$, V. Palladino, M. Pesaresi, D.M. Raymond, A. Richards, A. Rose, E. Scott, C. Seez, A. Shtipliyski, S. Summers, A. Tapper, K. Uchida, M. Vazquez Acosta ${ }^{64}$, T. Virdee ${ }^{13}$, D. Winterbottom, J. Wright, S.C. Zenz

\section{Brunel University, Uxbridge, United Kingdom}

J.E. Cole, P.R. Hobson, A. Khan, P. Kyberd, I.D. Reid, P. Symonds, L. Teodorescu, M. Turner

\section{Baylor University, Waco, U.S.A.}

A. Borzou, K. Call, J. Dittmann, K. Hatakeyama, H. Liu, N. Pastika, C. Smith

\section{Catholic University of America, Washington DC, U.S.A.}

R. Bartek, A. Dominguez

The University of Alabama, Tuscaloosa, U.S.A.

A. Buccilli, S.I. Cooper, C. Henderson, P. Rumerio, C. West

\section{Boston University, Boston, U.S.A.}

D. Arcaro, A. Avetisyan, T. Bose, D. Gastler, D. Rankin, C. Richardson, J. Rohlf, L. Sulak, D. Zou

\section{Brown University, Providence, U.S.A.}

G. Benelli, D. Cutts, A. Garabedian, J. Hakala, U. Heintz, J.M. Hogan, K.H.M. Kwok, E. Laird, G. Landsberg, Z. Mao, M. Narain, J. Pazzini, S. Piperov, S. Sagir, R. Syarif, D. $\mathrm{Yu}$

\section{University of California, Davis, Davis, U.S.A.}

R. Band, C. Brainerd, D. Burns, M. Calderon De La Barca Sanchez, M. Chertok, J. Conway, R. Conway, P.T. Cox, R. Erbacher, C. Flores, G. Funk, M. Gardner, W. Ko, R. Lander, C. Mclean, M. Mulhearn, D. Pellett, J. Pilot, S. Shalhout, M. Shi, J. Smith, M. Squires, D. Stolp, K. Tos, M. Tripathi, Z. Wang

\section{University of California, Los Angeles, U.S.A.}

M. Bachtis, C. Bravo, R. Cousins, A. Dasgupta, A. Florent, J. Hauser, M. Ignatenko, N. Mccoll, D. Saltzberg, C. Schnaible, V. Valuev

University of California, Riverside, Riverside, U.S.A.

E. Bouvier, K. Burt, R. Clare, J. Ellison, J.W. Gary, S.M.A. Ghiasi Shirazi, G. Hanson, J. Heilman, P. Jandir, E. Kennedy, F. Lacroix, O.R. Long, M. Olmedo Negrete, M.I. Paneva, A. Shrinivas, W. Si, L. Wang, H. Wei, S. Wimpenny, B. R. Yates

\section{University of California, San Diego, La Jolla, U.S.A.}

J.G. Branson, S. Cittolin, M. Derdzinski, B. Hashemi, A. Holzner, D. Klein, G. Kole, V. Krutelyov, J. Letts, I. Macneill, M. Masciovecchio, D. Olivito, S. Padhi, M. Pieri, 
M. Sani, V. Sharma, S. Simon, M. Tadel, A. Vartak, S. Wasserbaech ${ }^{65}$, J. Wood, F. Würthwein, A. Yagil, G. Zevi Della Porta

University of California, Santa Barbara - Department of Physics, Santa Barbara, U.S.A.

N. Amin, R. Bhandari, J. Bradmiller-Feld, C. Campagnari, A. Dishaw, V. Dutta, M. Franco Sevilla, C. George, F. Golf, L. Gouskos, J. Gran, R. Heller, J. Incandela, S.D. Mullin, A. Ovcharova, H. Qu, J. Richman, D. Stuart, I. Suarez, J. Yoo

California Institute of Technology, Pasadena, U.S.A.

D. Anderson, J. Bendavid, A. Bornheim, J.M. Lawhorn, H.B. Newman, T. Nguyen,

C. Pena, M. Spiropulu, J.R. Vlimant, S. Xie, Z. Zhang, R.Y. Zhu

Carnegie Mellon University, Pittsburgh, U.S.A.

M.B. Andrews, T. Ferguson, T. Mudholkar, M. Paulini, J. Russ, M. Sun, H. Vogel, I. Vorobiev, M. Weinberg

University of Colorado Boulder, Boulder, U.S.A.

J.P. Cumalat, W.T. Ford, F. Jensen, A. Johnson, M. Krohn, S. Leontsinis, T. Mulholland, K. Stenson, S.R. Wagner

Cornell University, Ithaca, U.S.A.

J. Alexander, J. Chaves, J. Chu, S. Dittmer, K. Mcdermott, N. Mirman, J.R. Patterson, A. Rinkevicius, A. Ryd, L. Skinnari, L. Soffi, S.M. Tan, Z. Tao, J. Thom, J. Tucker, P. Wittich, M. Zientek

\section{Fermi National Accelerator Laboratory, Batavia, U.S.A.}

S. Abdullin, M. Albrow, G. Apollinari, A. Apresyan, A. Apyan, S. Banerjee, L.A.T. Bauerdick, A. Beretvas, J. Berryhill, P.C. Bhat, G. Bolla, K. Burkett, J.N. Butler, A. Canepa, G.B. Cerati, H.W.K. Cheung, F. Chlebana, M. Cremonesi, J. Duarte, V.D. Elvira, J. Freeman, Z. Gecse, E. Gottschalk, L. Gray, D. Green, S. Grünendahl, O. Gutsche, R.M. Harris, S. Hasegawa, J. Hirschauer, Z. Hu, B. Jayatilaka, S. Jindariani, M. Johnson, U. Joshi, B. Klima, B. Kreis, S. Lammel, D. Lincoln, R. Lipton, M. Liu, T. Liu, R. Lopes De Sá, J. Lykken, K. Maeshima, N. Magini, J.M. Marraffino, S. Maruyama, D. Mason, P. McBride, P. Merkel, S. Mrenna, S. Nahn, V. O’Dell, K. Pedro, O. Prokofyev, G. Rakness, L. Ristori, B. Schneider, E. Sexton-Kennedy, A. Soha, W.J. Spalding, L. Spiegel, S. Stoynev, J. Strait, N. Strobbe, L. Taylor, S. Tkaczyk, N.V. Tran, L. Uplegger, E.W. Vaandering, C. Vernieri, M. Verzocchi, R. Vidal, M. Wang, H.A. Weber, A. Whitbeck

University of Florida, Gainesville, U.S.A.

D. Acosta, P. Avery, P. Bortignon, D. Bourilkov, A. Brinkerhoff, A. Carnes, M. Carver, D. Curry, S. Das, R.D. Field, I.K. Furic, J. Konigsberg, A. Korytov, K. Kotov, P. Ma, K. Matchev, H. Mei, G. Mitselmakher, D. Rank, D. Sperka, N. Terentyev, L. Thomas, J. Wang, S. Wang, J. Yelton

Florida International University, Miami, U.S.A.

Y.R. Joshi, S. Linn, P. Markowitz, J.L. Rodriguez 
Florida State University, Tallahassee, U.S.A.

A. Ackert, T. Adams, A. Askew, S. Hagopian, V. Hagopian, K.F. Johnson, T. Kolberg, G. Martinez, T. Perry, H. Prosper, A. Saha, A. Santra, R. Yohay

Florida Institute of Technology, Melbourne, U.S.A.

M.M. Baarmand, V. Bhopatkar, S. Colafranceschi, M. Hohlmann, D. Noonan, T. Roy, F. Yumiceva

University of Illinois at Chicago (UIC), Chicago, U.S.A.

M.R. Adams, L. Apanasevich, D. Berry, R.R. Betts, R. Cavanaugh, X. Chen, O. Evdokimov, C.E. Gerber, D.A. Hangal, D.J. Hofman, K. Jung, J. Kamin, I.D. Sandoval Gonzalez, M.B. Tonjes, H. Trauger, N. Varelas, H. Wang, Z. Wu, J. Zhang

The University of Iowa, Iowa City, U.S.A.

B. Bilki ${ }^{66}$, W. Clarida, K. Dilsiz ${ }^{67}$, S. Durgut, R.P. Gandrajula, M. Haytmyradov, V. Khristenko, J.-P. Merlo, H. Mermerkaya ${ }^{68}$, A. Mestvirishvili, A. Moeller, J. Nachtman, H. Ogul ${ }^{69}$, Y. Onel, F. Ozok ${ }^{70}$, A. Penzo, C. Snyder, E. Tiras, J. Wetzel, K. Yi

Johns Hopkins University, Baltimore, U.S.A.

B. Blumenfeld, A. Cocoros, N. Eminizer, D. Fehling, L. Feng, A.V. Gritsan, P. Maksimovic, J. Roskes, U. Sarica, M. Swartz, M. Xiao, C. You

The University of Kansas, Lawrence, U.S.A.

A. Al-bataineh, P. Baringer, A. Bean, S. Boren, J. Bowen, J. Castle, S. Khalil, A. Kropivnitskaya, D. Majumder, W. Mcbrayer, M. Murray, C. Royon, S. Sanders, E. Schmitz, R. Stringer, J.D. Tapia Takaki, Q. Wang

Kansas State University, Manhattan, U.S.A.

A. Ivanov, K. Kaadze, Y. Maravin, A. Mohammadi, L.K. Saini, N. Skhirtladze, S. Toda

Lawrence Livermore National Laboratory, Livermore, U.S.A.

F. Rebassoo, D. Wright

University of Maryland, College Park, U.S.A.

C. Anelli, A. Baden, O. Baron, A. Belloni, B. Calvert, S.C. Eno, C. Ferraioli, N.J. Hadley,

S. Jabeen, G.Y. Jeng, R.G. Kellogg, J. Kunkle, A.C. Mignerey, F. Ricci-Tam, Y.H. Shin,

A. Skuja, S.C. Tonwar

Massachusetts Institute of Technology, Cambridge, U.S.A.

D. Abercrombie, B. Allen, V. Azzolini, R. Barbieri, A. Baty, R. Bi, S. Brandt, W. Busza, I.A. Cali, M. D’Alfonso, Z. Demiragli, G. Gomez Ceballos, M. Goncharov, D. Hsu, Y. Iiyama, G.M. Innocenti, M. Klute, D. Kovalskyi, Y.S. Lai, Y.-J. Lee, A. Levin, P.D. Luckey, B. Maier, A.C. Marini, C. Mcginn, C. Mironov, S. Narayanan, X. Niu, C. Paus, C. Roland, G. Roland, J. Salfeld-Nebgen, G.S.F. Stephans, K. Tatar, D. Velicanu, J. Wang, T.W. Wang, B. Wyslouch

University of Minnesota, Minneapolis, U.S.A.

A.C. Benvenuti, R.M. Chatterjee, A. Evans, P. Hansen, S. Kalafut, Y. Kubota, Z. Lesko, J. Mans, S. Nourbakhsh, N. Ruckstuhl, R. Rusack, J. Turkewitz 
University of Mississippi, Oxford, U.S.A.

J.G. Acosta, S. Oliveros

University of Nebraska-Lincoln, Lincoln, U.S.A.

E. Avdeeva, K. Bloom, D.R. Claes, C. Fangmeier, R. Gonzalez Suarez, R. Kamalieddin, I. Kravchenko, J. Monroy, J.E. Siado, G.R. Snow, B. Stieger

State University of New York at Buffalo, Buffalo, U.S.A.

M. Alyari, J. Dolen, A. Godshalk, C. Harrington, I. Iashvili, D. Nguyen, A. Parker, S. Rappoccio, B. Roozbahani

Northeastern University, Boston, U.S.A.

G. Alverson, E. Barberis, A. Hortiangtham, A. Massironi, D.M. Morse, D. Nash, T. Orimoto, R. Teixeira De Lima, D. Trocino, D. Wood

Northwestern University, Evanston, U.S.A.

S. Bhattacharya, O. Charaf, K.A. Hahn, N. Mucia, N. Odell, B. Pollack, M.H. Schmitt, K. Sung, M. Trovato, M. Velasco

University of Notre Dame, Notre Dame, U.S.A.

N. Dev, M. Hildreth, K. Hurtado Anampa, C. Jessop, D.J. Karmgard, N. Kellams, K. Lannon, N. Loukas, N. Marinelli, F. Meng, C. Mueller, Y. Musienko ${ }^{35}$, M. Planer, A. Reinsvold, R. Ruchti, G. Smith, S. Taroni, M. Wayne, M. Wolf, A. Woodard

The Ohio State University, Columbus, U.S.A.

J. Alimena, L. Antonelli, B. Bylsma, L.S. Durkin, S. Flowers, B. Francis, A. Hart, C. Hill, W. Ji, B. Liu, W. Luo, D. Puigh, B.L. Winer, H.W. Wulsin

Princeton University, Princeton, U.S.A.

A. Benaglia, S. Cooperstein, O. Driga, P. Elmer, J. Hardenbrook, P. Hebda, S. Higginbotham, D. Lange, J. Luo, D. Marlow, K. Mei, I. Ojalvo, J. Olsen, C. Palmer, P. Piroué, D. Stickland, C. Tully

University of Puerto Rico, Mayaguez, U.S.A.

S. Malik, S. Norberg

Purdue University, West Lafayette, U.S.A.

A. Barker, V.E. Barnes, S. Folgueras, L. Gutay, M.K. Jha, M. Jones, A.W. Jung, A. Khatiwada, D.H. Miller, N. Neumeister, C.C. Peng, J.F. Schulte, J. Sun, F. Wang, W. Xie

Purdue University Northwest, Hammond, U.S.A.

T. Cheng, N. Parashar, J. Stupak

Rice University, Houston, U.S.A.

A. Adair, B. Akgun, Z. Chen, K.M. Ecklund, F.J.M. Geurts, M. Guilbaud, M. Kilpatrick, W. Li, B. Michlin, B.P. Padley, J. Roberts, J. Rorie, Z. Tu, J. Zabel 
University of Rochester, Rochester, U.S.A.

A. Bodek, P. de Barbaro, R. Demina, Y.t. Duh, T. Ferbel, M. Galanti, A. Garcia-Bellido, J. Han, O. Hindrichs, A. Khukhunaishvili, K.H. Lo, P. Tan, M. Verzetti

The Rockefeller University, New York, U.S.A.

R. Ciesielski, K. Goulianos, C. Mesropian

Rutgers, The State University of New Jersey, Piscataway, U.S.A.

A. Agapitos, J.P. Chou, Y. Gershtein, T.A. Gómez Espinosa, E. Halkiadakis, M. Heindl, E. Hughes, S. Kaplan, R. Kunnawalkam Elayavalli, S. Kyriacou, A. Lath, R. Montalvo, K. Nash, M. Osherson, H. Saka, S. Salur, S. Schnetzer, D. Sheffield, S. Somalwar, R. Stone, S. Thomas, P. Thomassen, M. Walker

University of Tennessee, Knoxville, U.S.A.

A.G. Delannoy, M. Foerster, J. Heideman, G. Riley, K. Rose, S. Spanier, K. Thapa

Texas A\&M University, College Station, U.S.A.

O. Bouhali ${ }^{71}$, A. Castaneda Hernandez ${ }^{71}$, A. Celik, M. Dalchenko, M. De Mattia, A. Delgado, S. Dildick, R. Eusebi, J. Gilmore, T. Huang, T. Kamon ${ }^{72}$, R. Mueller, Y. Pakhotin, R. Patel, A. Perloff, L. Perniè, D. Rathjens, A. Safonov, A. Tatarinov, K.A. Ulmer

Texas Tech University, Lubbock, U.S.A.

N. Akchurin, J. Damgov, F. De Guio, P.R. Dudero, J. Faulkner, E. Gurpinar, S. Kunori, K. Lamichhane, S.W. Lee, T. Libeiro, T. Peltola, S. Undleeb, I. Volobouev, Z. Wang

Vanderbilt University, Nashville, U.S.A.

S. Greene, A. Gurrola, R. Janjam, W. Johns, C. Maguire, A. Melo, H. Ni, P. Sheldon, S. Tuo, J. Velkovska, Q. Xu

\section{University of Virginia, Charlottesville, U.S.A.}

M.W. Arenton, P. Barria, B. Cox, R. Hirosky, A. Ledovskoy, H. Li, C. Neu, T. Sinthuprasith, X. Sun, Y. Wang, E. Wolfe, F. Xia

Wayne State University, Detroit, U.S.A.

R. Harr, P.E. Karchin, J. Sturdy, S. Zaleski

University of Wisconsin - Madison, Madison, WI, U.S.A.

M. Brodski, J. Buchanan, C. Caillol, S. Dasu, L. Dodd, S. Duric, B. Gomber, M. Grothe, M. Herndon, A. Hervé, U. Hussain, P. Klabbers, A. Lanaro, A. Levine, K. Long, R. Loveless, G.A. Pierro, G. Polese, T. Ruggles, A. Savin, N. Smith, W.H. Smith, D. Taylor, N. Woods

$\dagger$ : Deceased

1: Also at Vienna University of Technology, Vienna, Austria

2: Also at State Key Laboratory of Nuclear Physics and Technology, Peking University, Beijing, China

3: Also at Universidade Estadual de Campinas, Campinas, Brazil

4: Also at Universidade Federal de Pelotas, Pelotas, Brazil 
5: Also at Université Libre de Bruxelles, Bruxelles, Belgium

6: Also at Institute for Theoretical and Experimental Physics, Moscow, Russia

7: Also at Joint Institute for Nuclear Research, Dubna, Russia

8: Now at Ain Shams University, Cairo, Egypt

9: Now at British University in Egypt, Cairo, Egypt

10: Also at Zewail City of Science and Technology, Zewail, Egypt

11: Also at Université de Haute Alsace, Mulhouse, France

12: Also at Skobeltsyn Institute of Nuclear Physics, Lomonosov Moscow State University, Moscow, Russia

13: Also at CERN, European Organization for Nuclear Research, Geneva, Switzerland

14: Also at RWTH Aachen University, III. Physikalisches Institut A, Aachen, Germany

15: Also at University of Hamburg, Hamburg, Germany

16: Also at Brandenburg University of Technology, Cottbus, Germany

17: Also at Institute of Nuclear Research ATOMKI, Debrecen, Hungary

18: Also at MTA-ELTE Lendület CMS Particle and Nuclear Physics Group, Eötvös Loránd University, Budapest, Hungary

19: Also at Institute of Physics, University of Debrecen, Debrecen, Hungary

20: Also at Indian Institute of Technology Bhubaneswar, Bhubaneswar, India

21: Also at Institute of Physics, Bhubaneswar, India

22: Also at University of Visva-Bharati, Santiniketan, India

23: Also at University of Ruhuna, Matara, Sri Lanka

24: Also at Isfahan University of Technology, Isfahan, Iran

25: Also at Yazd University, Yazd, Iran

26: Also at Plasma Physics Research Center, Science and Research Branch, Islamic Azad University, Tehran, Iran

27: Also at Università degli Studi di Siena, Siena, Italy

28: Also at INFN Sezione di Milano-Bicocca; Università di Milano-Bicocca, Milano, Italy

29: Also at Laboratori Nazionali di Legnaro dell'INFN, Legnaro, Italy

30: Also at Purdue University, West Lafayette, U.S.A.

31: Also at International Islamic University of Malaysia, Kuala Lumpur, Malaysia

32: Also at Malaysian Nuclear Agency, MOSTI, Kajang, Malaysia

33: Also at Consejo Nacional de Ciencia y Tecnología, Mexico city, Mexico

34: Also at Warsaw University of Technology, Institute of Electronic Systems, Warsaw, Poland

35: Also at Institute for Nuclear Research, Moscow, Russia

36: Now at National Research Nuclear University 'Moscow Engineering Physics Institute' (MEPhI), Moscow, Russia

37: Also at St. Petersburg State Polytechnical University, St. Petersburg, Russia

38: Also at University of Florida, Gainesville, U.S.A.

39: Also at P.N. Lebedev Physical Institute, Moscow, Russia

40: Also at California Institute of Technology, Pasadena, U.S.A.

41: Also at Budker Institute of Nuclear Physics, Novosibirsk, Russia

42: Also at Faculty of Physics, University of Belgrade, Belgrade, Serbia

43: Also at INFN Sezione di Roma; Sapienza Università di Roma, Rome, Italy

44: Also at University of Belgrade, Faculty of Physics and Vinca Institute of Nuclear Sciences, Belgrade, Serbia

45: Also at Scuola Normale e Sezione dell'INFN, Pisa, Italy

46: Also at National and Kapodistrian University of Athens, Athens, Greece

47: Also at Riga Technical University, Riga, Latvia 
48: Also at Universität Zürich, Zurich, Switzerland

49: Also at Stefan Meyer Institute for Subatomic Physics (SMI), Vienna, Austria

50: Also at Istanbul University, Faculty of Science, Istanbul, Turkey

51: Also at Gaziosmanpasa University, Tokat, Turkey

52: Also at Adiyaman University, Adiyaman, Turkey

53: Also at Istanbul Aydin University, Istanbul, Turkey

54: Also at Mersin University, Mersin, Turkey

55: Also at Cag University, Mersin, Turkey

56: Also at Piri Reis University, Istanbul, Turkey

57: Also at Izmir Institute of Technology, Izmir, Turkey

58: Also at Necmettin Erbakan University, Konya, Turkey

59: Also at Marmara University, Istanbul, Turkey

60: Also at Kafkas University, Kars, Turkey

61: Also at Istanbul Bilgi University, Istanbul, Turkey

62: Also at Rutherford Appleton Laboratory, Didcot, United Kingdom

63: Also at School of Physics and Astronomy, University of Southampton, Southampton, United Kingdom

64: Also at Instituto de Astrofísica de Canarias, La Laguna, Spain

65: Also at Utah Valley University, Orem, U.S.A.

66: Also at Beykent University, Istanbul, Turkey

67: Also at Bingol University, Bingol, Turkey

68: Also at Erzincan University, Erzincan, Turkey

69: Also at Sinop University, Sinop, Turkey

70: Also at Mimar Sinan University, Istanbul, Istanbul, Turkey

71: Also at Texas A\&M University at Qatar, Doha, Qatar

72: Also at Kyungpook National University, Daegu, Korea 